TRANSACTIONS OF THE

AMERICAN MATHEMATICAL SOCIETY

Volume 356, Number 11, Pages 4287-4322

S 0002-9947(04)03636-0

Article electronically published on May 28, 2004

\title{
A CLASS OF $C^{*}$-ALGEBRAS GENERALIZING BOTH GRAPH ALGEBRAS AND HOMEOMORPHISM $C^{*}$-ALGEBRAS I, FUNDAMENTAL RESULTS
}

\author{
TAKESHI KATSURA
}

\begin{abstract}
We introduce a new class of $C^{*}$-algebras, which is a generalization of both graph algebras and homeomorphism $C^{*}$-algebras. This class is very large and also very tractable. We prove the so-called gauge-invariant uniqueness theorem and the Cuntz-Krieger uniqueness theorem, and compute the $K$-groups of our algebras.
\end{abstract}

\section{INTRODUCTION}

The purpose of this serial work is an introduction of a new class of $C^{*}$-algebras which contains graph algebras and homeomorphism $C^{*}$-algebras. Our class is very large so that it contains every AF-algebra $\mathrm{Ka} 2$ and every Kirchberg algebra satisfying the UCT [Ka4] as well as many simple stably projectionless $C^{*}$-algebras. At the same time, our class can be well studied by using similar techniques developed in the analysis of graph algebras and homeomorphism $C^{*}$-algebras.

Since J. Cuntz and W. Krieger introduced a class of $C^{*}$-algebras arising from finite matrices with entries $\{0,1\}$ in [CK], there have been many generalizations of Cuntz-Krieger algebras, for example, Exel-Laca algebras [EL], graph algebras KPRR, KPR [FLR and Cuntz-Pimsner algebras $[\mathrm{P}]$. Among others, investigation of graph algebras has rapidly progressed these days (see, for example, BPRS. BHRS, [HS, DT1]), and many structures of graph algebras have been characterized in terms of graphs. As some authors pointed out, it is time to extend the techniques and results on graph algebras to more general $C^{*}$-algebras. Our work is one of such attempts. The investigation of homeomorphism $C^{*}$-algebras has also been developed mainly by J. Tomiyama [T1, T2, T3, T4]. These two lines of research have several similar aspects in common, and our aim in this series of work is to combine and unify these studies in the two active fields.

In this paper, we associate a $C^{*}$-algebra with a quadruple $E=\left(E^{0}, E^{1}, d, r\right)$ where $E^{0}$ and $E^{1}$ are locally compact spaces, $d: E^{1} \rightarrow E^{0}$ is a local homeomorphism, and $r: E^{1} \rightarrow E^{0}$ is a continuous map. A quadruple $E=\left(E^{0}, E^{1}, d, r\right)$ is called a topological graph. Note that when $E^{0}$ is a discrete set, this quadruple is an ordinary (directed) graph and the $C^{*}$-algebra constructed here is a graph algebra of it (or its opposite graph). In [D], V. Deaconu introduced a notion of compact graphs and associated $C^{*}$-algebras with them. Compact graphs are particular examples of

Received by the editors October 1, 2002

2000 Mathematics Subject Classification. Primary 46L05; Secondary 46L55, 37 B99.

(C)2004 American Mathematical Society 
topological graphs, and his $C^{*}$-algebras are isomorphic to ours. A triple $\left(E^{1}, d, r\right)$ can be considered as a generalization of continuous maps from $E^{0}$ to itself, and so a quadruple $E=\left(E^{0}, E^{1}, d, r\right)$ can be considered as a generalization of dynamical systems. This point of view is essential for the analysis of our $C^{*}$-algebras, and we borrow many notions from the theory of dynamical systems (see [Ka3]).

In the first paper of our serial work, we give a definition of our algebras and prove fundamental results on them. We first construct $C^{*}$-correspondences from topological graphs. This is done in Section 1 in a slightly more general form. Then in Section 2, we associate $C^{*}$-algebras with $C^{*}$-correspondences constructed from topological graphs, in a similar way to Cuntz-Pimsner algebras $[\mathrm{P}$. We, however, point out two distinctions between our approach and the one for Cuntz-Pimsner algebras (see also the end of Section 3 in this paper). The first point is that left actions of our $C^{*}$-correspondences may not be injective. This is not allowed in P because Cuntz-Pimsner algebras of $C^{*}$-correspondences with non-injective left actions often become zero (see $[\mathrm{P}$. Remark $1.2(1)]$ ). Note that our algebras are obtained as relative Cuntz-Pimsner algebras introduced in [MS]. The other point is that we can examine our algebras in more detail than Cuntz-Pimsner algebras. This is because our algebras are defined from "topological" data, whereas Cuntz-Pimsner algebras are arising from arbitrary $C^{*}$-correspondences. In Section 3, we give one concrete representation of our algebras by using so-called Fock spaces. In Sections 4 and 5 , we prove two fundamental theorems, namely the gauge-invariant uniqueness theorem (Theorem 4.5) and the Cuntz-Krieger uniqueness theorem (Theorem 5.12). Both of these theorems were known for graph algebras [BPRS, RaSz, DT1, BHRS] and for homeomorphism $C^{*}$-algebras [T1, T2, T4] (see also [ELQ]). We give a unified approach to these two active branches. In the final section, we prove that our algebras are always nuclear and satisfy the universal coefficient theorem (UCT), and give six-term exact sequences of $K$-groups which are useful to compute $K$ groups of our algebras. In particular, this gives a new proof for the computation of $K$-groups of graph algebras.

We remark the following about our notation, which is different from other articles. We use $d$ and $r$ for denoting the "domain" map and the "range" map of topological graphs. These terms suit well when we consider quadruples as generalization of dynamical systems. However, our convention is opposite to the one used in many papers on graph algebras (for example, [KPRR, KPR [FLR]). The author believes that even for graph algebras of discrete graphs, our convention is more natural than the one used in many papers on graph algebras. One of the reasons is that our convention behaves well when one considers graphs as a kind of dynamical systems and graph algebras as crossed products of them. Another reason is that under our convention, the definition of Toeplitz families (or Cuntz-Krieger families) $\left\{S_{e}\right\},\left\{P_{v}\right\}$ satisfies that an initial projection of $S_{e}$ for an edge $e$ is the projection $P_{d(e)}$ for the domain $d(e)$ of $e$, and a range projection of $S_{e}$ is a subprojection of $P_{r(e)}$ for the range $r(e)$ of $e$. Note that the strangeness of our definition of paths comes from the order of the compositions of two maps (see Section 2). The author is grateful to Ruy Exel for encouraging him to adopt this convention.

We denote the set of natural numbers by $\mathbb{N}=\{0,1,2, \ldots\}$ and the set of complex numbers by $\mathbb{C}$. We denote by $\mathbb{T}$ the group consisting of complex numbers whose absolute values are 1 . For a locally compact (Hausdorff) space $X$, we denote by $C(X)$ the linear space of all continuous functions on $X$. We define three subspaces 
$C_{c}(X), C_{0}(X)$ and $C_{b}(X)$ of $C(X)$ by those of compactly supported functions, functions vanishing at infinity, and bounded functions, respectively.

\section{Topological CORRESPONDENCES AND $C^{*}$-CORRESPONDENCES}

In this section, we introduce a notion of topological correspondences and give a method to construct $C^{*}$-correspondences from them. This method had already appeared in D, DKM] Sc or other papers. They used a completion procedure to get $C^{*}$-correspondences. However we need a concrete description of our $C^{*}$ correspondences.

A (right) Hilbert $A$-module $X$ is a Banach space with a right action of a $C^{*}$ algebra $A$ and an $A$-valued inner product $\langle\cdot, \cdot\rangle$ satisfying

(i) $\langle\xi, \eta f\rangle=\langle\xi, \eta\rangle f$,

(ii) $\langle\xi, \eta\rangle=\langle\eta, \xi\rangle^{*}$,

(iii) $\langle\xi, \xi\rangle \geq 0$ and $\|\xi\|=\|\langle\xi, \xi\rangle\|^{1 / 2}$,

for $\xi, \eta \in X$ and $f \in A$ (for the detail, see $[\mathrm{L}$ ). For a Hilbert $A$-module $X$, we denote by $\mathcal{L}(X)$ the $C^{*}$-algebra of all adjointable operators on $X$. For $\xi, \eta \in X$, the operator $\theta_{\xi, \eta} \in \mathcal{L}(X)$ is defined by $\theta_{\xi, \eta}(\zeta)=\xi\langle\eta, \zeta\rangle$ for $\zeta \in X$. By definition,

$$
\mathcal{K}(X)=\overline{\operatorname{span}}\left\{\theta_{\xi, \eta} \mid \xi, \eta \in X\right\}
$$

which is an ideal of $\mathcal{L}(X)$. For $C^{*}$-algebras $A, B$, we say that $X$ is a $C^{*}$-correspondence from $A$ to $B$ when $X$ is a Hilbert $B$-module and a left action $\pi$ of $A$ on $X$, which is just a $*$-homomorphism $\pi: A \rightarrow \mathcal{L}(X)$, is given. A $C^{*}$-correspondence from $A$ to itself is called a $C^{*}$-correspondence over $A$. When a $C^{*}$-correspondence $X$ from $A$ to $B$ and a $C^{*}$-correspondence $Y$ from $B$ to $C$ are given, we can define the interior tensor product $X \otimes Y$, which is a $C^{*}$-correspondence from $A$ to $C$, as follows. The algebraic tensor product $X \odot_{B} Y$ over $B$ is, by definition, a quotient of the algebraic tensor product $X \odot Y$ (as a $\mathbb{C}$-vector space) by the subspace generated by $(\xi f) \otimes \eta-\xi \otimes\left(\pi_{Y}(f) \eta\right)$ for $\xi \in X, \eta \in Y, f \in B$, where $\pi_{Y}: B \rightarrow \mathcal{L}(Y)$ is the given left action. The image of $\xi \otimes \eta \in X \odot Y$ in $X \odot_{B} Y$ is also denoted by $\xi \otimes \eta$. We define a left action $\pi$ of $A$, a right action of $C$ and a $C$-valued inner product on $X \odot_{B} Y$ by

$$
\begin{aligned}
\pi(f)(\xi \otimes \eta) & =\left(\pi_{X}(f) \xi\right) \otimes \eta, \\
(\xi \otimes \eta) g & =\xi \otimes(\eta g), \\
\left\langle\xi \otimes \eta, \xi^{\prime} \otimes \eta^{\prime}\right\rangle & =\left\langle\eta, \pi_{Y}\left(\left\langle\xi, \xi^{\prime}\right\rangle\right) \eta^{\prime}\right\rangle
\end{aligned}
$$

for $\xi, \xi^{\prime} \in X, \eta, \eta^{\prime} \in Y, f \in A, g \in C$. One can show that these operations are well defined and extend to the completion of $X \odot_{B} Y$ with respect to the norm coming from the $C$-valued inner product defined above (see $[\mathrm{L}$, Proposition 4.5]). Thus the completion of $X \odot_{B} Y$ is a $C^{*}$-correspondence from $A$ to $C$. This $C^{*}$-correspondence is the interior tensor product of $X$ and $Y$, and denoted by $X \otimes Y$.

Definition 1.1. Let $E^{0}$ and $E^{1}$ be locally compact (Hausdorff) spaces. A map $d: E^{1} \rightarrow E^{0}$ is said to be locally homeomorphic if for any $e \in E^{1}$, there exists a neighborhood $U$ of $e$ such that the restriction of $d$ to $U$ is a homeomorphism onto $d(U)$ and that $d(U)$ is a neighborhood of $d(e)$.

Every local homeomorphism is continuous and open. If $E^{0}$ is discrete and there exists a local homeomorphism $d: E^{1} \rightarrow E^{0}$, then $E^{1}$ is also discrete. 
Definition 1.2. Let $E^{0}$ and $F^{0}$ be locally compact spaces. A topological correspondence from $E^{0}$ to $F^{0}$ is a triple $\left(E^{1}, d, r\right)$ where $E^{1}$ is a locally compact space, $d: E^{1} \rightarrow E^{0}$ is a local homeomorphism and $r: E^{1} \rightarrow F^{0}$ is a continuous map.

When both $d$ and $r$ are surjective local homeomorphisms, $\left(E^{1}, d, r\right)$ is called a polymorphism in [AR]. A continuous map $\varphi: E^{0} \rightarrow F^{0}$ gives an example of topological correspondence $\left(E^{0}\right.$, id,$\left.\varphi\right)$. More generally, a set of continuous maps from (possibly infinitely many) open subsets $O_{i}$ of $E^{0}$ to $F^{0}$ gives a topological correspondence by setting $E^{1}=\coprod_{i} O_{i}$ and defining $d$ by natural inclusions. Thus we consider a topological correspondence as a generalization of (many-valued) continuous maps. The pair $\left(E^{1}, d\right)$ defines a "domain" of a topological correspondence $\left(E^{1}, d, r\right)$. "Locally" we can define a homeomorphism $d^{-1}$ from an open subset of $E^{0}$ to an open subset of $E^{1}$, and $r \circ d^{-1}$ gives a continuous map from an open subset of $E^{0}$ to $F^{0}$. The "image" of a point $v \in E^{0}$ under the topological correspondence $\left(E^{1}, d, r\right)$ is $r\left(d^{-1}(v)\right) \subset F^{0}$, which can be more than one point (possibly infinitely many points) or empty. The "inverse image" of an open subset $V$ of $F^{0}$ is $d\left(r^{-1}(V)\right)$, which is an open subset of $E^{0}$. When $E^{0}$ and $F^{0}$ are discrete, a topological correspondence $\left(E^{1}, d, r\right)$ is just a directed graph from $E^{0}$ to $F^{0}$.

Throughout this section, we fix locally compact spaces $E^{0}, F^{0}$ and a topological correspondence $\left(E^{1}, d, r\right)$ from $E^{0}$ to $F^{0}$. We will see that the topological correspondence $\left(E^{1}, d, r\right)$ naturally defines a $C^{*}$-correspondence $C_{d}\left(E^{1}\right)$ from $C_{0}\left(F^{0}\right)$ to $C_{0}\left(E^{0}\right)$. First we define a Hilbert $C_{0}\left(E^{0}\right)$-module $C_{d}\left(E^{1}\right)$ by using the data of the "domain" $\left(E^{1}, d\right)$. For $\xi \in C\left(E^{1}\right)$, we define a map $\langle\xi, \xi\rangle: E^{0} \rightarrow[0, \infty]$ by $\langle\xi, \xi\rangle(v)=\sum_{e \in d^{-1}(v)}|\xi(e)|^{2}$ for $v \in E^{0}$, and set $\|\xi\|=\sup _{v \in E^{0}}\langle\xi, \xi\rangle(v)^{1 / 2}$. We define

$$
C_{d}\left(E^{1}\right)=\left\{\xi \in C\left(E^{1}\right) \mid\langle\xi, \xi\rangle \in C_{0}\left(E^{0}\right)\right\} .
$$

Note that we have $\|\xi\|<\infty$ for $\xi \in C_{d}\left(E^{1}\right)$. We will show that $C_{d}\left(E^{1}\right)$ is a Hilbert $C_{0}\left(E^{0}\right)$-module. For $\xi, \eta \in C_{d}\left(E^{1}\right)$, we define $\langle\xi, \eta\rangle: E^{0} \rightarrow \mathbb{C}$ by

$$
\langle\xi, \eta\rangle(v)=\sum_{e \in d^{-1}(v)} \overline{\xi(e)} \eta(e) \in \mathbb{C}
$$

for $v \in E^{0}$. This is well defined because $\langle\xi, \xi\rangle(v),\langle\eta, \eta\rangle(v)<\infty$. To prove that $C_{d}\left(E^{1}\right)$ is a linear space, we need to prove $\langle\xi, \eta\rangle \in C_{0}\left(E^{0}\right)$ for $\xi, \eta \in C_{d}\left(E^{1}\right)$. First we show that $\langle\xi, \eta\rangle \in C_{0}\left(E^{0}\right)$ for $\xi, \eta \in C_{c}\left(E^{1}\right)$.

Lemma 1.3. For any $v \in E^{0}$, the set $d^{-1}(v)$ has no accumulation points.

Proof. If $d^{-1}(v)$ has an accumulation point $e \in E^{1}$, then $\left.d\right|_{U}: U \rightarrow d(U)$ is not injective for any neighborhood $U$ of $e$. It contradicts the fact that $d$ is locally homeomorphic.

Lemma 1.4. For any compact subset $X$ of $E^{1}$ and any $v \in E^{0}$, there exist an open neighborhood $V$ of $v$ and mutually disjoint open sets $U_{1}, \ldots, U_{n}$ of $E^{1}$ such that the restriction of $d$ to $U_{k}$ is a homeomorphism onto $V$ for each $k=1, \ldots, n$ and that $X \cap d^{-1}(V) \subset \bigcup_{k=1}^{n} U_{k}$.

Proof. Since $X$ is compact, $d^{-1}(v) \cap X$ is a finite set $\left\{e_{1}, \ldots, e_{n}\right\}$ by Lemma 1.3. Since $d$ is a local homeomorphism, there exist a neighborhood $V^{\prime}$ of $v$ and a neighborhood $U_{k}^{\prime}$ of $e_{k} \in E^{1}$ for each $k$ such that the restriction of $d$ to $U_{k}^{\prime}$ is a homeomorphism onto $V^{\prime}$. We may assume that $U_{k}^{\prime}$ 's are mutually disjoint. We will show that there exists a neighborhood $V$ of $v$ such that $V \subset V^{\prime}$ and 
$X \cap d^{-1}(V) \subset \bigcup_{k=1}^{n} U_{k}^{\prime}$. To the contrary, assume that for each $V \subset V^{\prime}$ there exists $e_{V} \in X \cap d^{-1}(V)$ with $e_{V} \notin \bigcup_{k=1}^{n} U_{k}^{\prime}$. Since $X$ is compact, we can find a subnet $\left\{e_{V_{\lambda}}\right\}_{\lambda \in \Lambda}$ of the net $\left\{e_{V}\right\}_{V \subset V^{\prime}}$ which converges to some element $e \in X$. We see that $d(e)=\lim _{\lambda} d\left(e_{V_{\lambda}}\right)=\lim _{V} d\left(e_{V}\right)=v$. Hence we have $e=e_{k}$ for some $k \in\{1, \ldots, n\}$. Then we can find $\lambda \in \Lambda$ with $e_{V_{\lambda}} \in U_{k}^{\prime}$. This is a contradiction. Thus we can find a neighborhood $V$ of $v$ such that $V \subset V^{\prime}$ and

$$
X \cap d^{-1}(V) \subset\left(\bigcup_{k=1}^{n} U_{k}^{\prime}\right) \cap d^{-1}(V) .
$$

Then $V$ and $U_{k}=U_{k}^{\prime} \cap d^{-1}(V)$ are the desired sets.

Lemma 1.5. For $\xi, \eta \in C_{c}\left(E^{1}\right)$, we have $\langle\xi, \eta\rangle \in C_{c}\left(E^{0}\right)$.

Proof. Lemma 1.3 ensures that for each $v \in E^{0}, \overline{\xi(e)} \eta(e)=0$ for all but finite $e \in d^{-1}(v)$. Hence we can define $\langle\xi, \eta\rangle(v)$ for $v \in E^{0}$, and we have $\langle\xi, \eta\rangle \in C_{c}\left(E^{0}\right)$ by Lemma 1.4

By taking $\xi=\eta$ in Lemma 1.5 we have $C_{c}\left(E^{1}\right) \subset C_{d}\left(E^{1}\right)$.

Lemma 1.6. For any $\xi \in C_{d}\left(E^{1}\right)$ and any $\varepsilon>0$, there exists $\eta \in C_{c}\left(E^{1}\right)$ such that $\|\eta\| \leq\|\xi\|$ and $\|\xi-\eta\|<\varepsilon$.

Proof. Since $\langle\xi, \xi\rangle \in C_{0}\left(E^{0}\right)$, there exists a compact subset $Y$ of $E^{0}$ such that $\langle\xi, \xi\rangle(v)<\varepsilon^{2}$ for $v \notin Y$. Take $v \in Y$. We will show that there exist a neighborhood $V_{v}$ of $v$ and a compact subset $X_{v}$ of $E^{1}$ such that

$$
\sum_{e \in d^{-1}\left(v^{\prime}\right) \backslash X_{v}}|\xi(e)|^{2}<\varepsilon^{2}
$$

for all $v^{\prime} \in V_{v}$. Since $\langle\xi, \xi\rangle(v)=\sum_{e \in d^{-1}(v)}|\xi(e)|^{2}<\infty$, there exist $e_{1}, \ldots, e_{n} \in$ $d^{-1}(v)$ such that

$$
\langle\xi, \xi\rangle(v)-\sum_{k=1}^{n}\left|\xi\left(e_{k}\right)\right|^{2}<\frac{\varepsilon^{2}}{3} .
$$

For each $k=1, \ldots, n$, we can find a compact neighborhood $U_{k}$ of $e_{k}$ such that the restriction of $d$ to $U_{k}$ is injective and $\left.|| \xi\left(e_{k}\right)\right|^{2}-|\xi(e)|^{2} \mid<\varepsilon^{2} / 3 n$ for $e \in U_{k}$. By replacing $U_{k}$ 's by smaller sets if necessary, we may assume that $U_{k} \cap U_{l}=\emptyset$ for $k \neq l$. Since $d$ is a local homeomorphism, $\bigcap_{k=1}^{n} d\left(U_{k}\right)$ is a neighborhood of $v$. Hence we can find a neighborhood $V_{v}$ of $v$ such that $V_{v} \subset \bigcap_{k=1}^{n} d\left(U_{k}\right)$ and $\left|\langle\xi, \xi\rangle\left(v^{\prime}\right)-\langle\xi, \xi\rangle(v)\right|<\varepsilon^{2} / 3$ for $v^{\prime} \in V_{v}$. Set $X_{v}=\bigcup_{k=1}^{n} U_{k}$ which is a compact subset of $E^{1}$. For $v^{\prime} \in V_{v}$, there exists a unique element $e_{k}^{\prime} \in U_{k}$ with $d\left(e_{k}^{\prime}\right)=v^{\prime}$. We have

$$
\begin{aligned}
\sum_{e \in d^{-1}\left(v^{\prime}\right) \backslash X_{v}}|\xi(e)|^{2} & =\langle\xi, \xi\rangle\left(v^{\prime}\right)-\sum_{e \in d^{-1}\left(v^{\prime}\right) \cap X_{v}}|\xi(e)|^{2} \\
& \leq\left|\langle\xi, \xi\rangle\left(v^{\prime}\right)-\langle\xi, \xi\rangle(v)\right|+\left.\left|\langle\xi, \xi\rangle(v)-\sum_{k=1}^{n}\right| \xi\left(e_{k}^{\prime}\right)\right|^{2} \mid \\
& <\frac{\varepsilon^{2}}{3}+\left.\left|\langle\xi, \xi\rangle(v)-\sum_{k=1}^{n}\right| \xi\left(e_{k}\right)\right|^{2}\left|+\sum_{k=1}^{n}\right|\left|\xi\left(e_{k}\right)\right|^{2}-\left|\xi\left(e_{k}^{\prime}\right)\right|^{2} \mid \\
& <\frac{\varepsilon^{2}}{3}+\frac{\varepsilon^{2}}{3}+n \frac{\varepsilon^{2}}{3 n}=\varepsilon^{2}
\end{aligned}
$$


Hence we have found a neighborhood $V_{v}$ of $v$ and a compact subset $X_{v}$ of $E^{1}$ such that

$$
\sum_{e \in d^{-1}\left(v^{\prime}\right) \backslash X_{v}}|\xi(e)|^{2}<\varepsilon^{2}
$$

for all $v^{\prime} \in V_{v}$. Since $Y$ is compact, there exist $v_{1}, \ldots, v_{m} \in Y$ such that $Y \subset$ $\bigcup_{k=1}^{m} V_{v_{k}}$. Set $X=\bigcup_{k=1}^{m} X_{v_{k}}$ which is a compact subset of $E^{1}$. We can find $\eta^{\prime} \in C_{c}\left(E^{1}\right)$ such that $0 \leq \eta^{\prime} \leq 1$ and $\eta^{\prime}(e)=1$ for $e \in X$. We set $\eta=\eta^{\prime} \xi \in$ $C_{c}\left(E^{1}\right)$. Then we have $|\eta(e)| \leq|\xi(e)|$. Hence we get $\|\eta\| \leq\|\xi\|$. We will prove $\|\xi-\eta\|<\varepsilon$ which completes the proof. Note that $(\xi-\eta)(e)=0$ for $e \in X$ and $|(\xi-\eta)(e)| \leq|\xi(e)|$ for all $e \in E^{1}$. For $v \notin Y$, we have

$$
\langle\xi-\eta, \xi-\eta\rangle(v) \leq\langle\xi, \xi\rangle(v)<\varepsilon^{2} .
$$

For $v \in Y$, we can find $k$ with $v \in V_{v_{k}}$. Hence we have

$$
\begin{aligned}
\langle\xi-\eta, \xi-\eta\rangle(v) & =\sum_{e \in d^{-1}(v)}|(\xi-\eta)(e)|^{2}=\sum_{e \in d^{-1}(v) \backslash X}|(\xi-\eta)(e)|^{2} \\
& \leq \sum_{e \in d^{-1}(v) \backslash X}|\xi(e)|^{2} \leq \sum_{e \in d^{-1}(v) \backslash X_{v_{k}}}|\xi(e)|^{2}<\varepsilon^{2} .
\end{aligned}
$$

Therefore we have $\|\xi-\eta\|<\varepsilon$. We are done.

By Lemma 1.6 we see that the linear space $C_{c}\left(E^{1}\right)$ is dense in $C_{d}\left(E^{1}\right)$.

Lemma 1.7. For $\xi, \eta \in C_{d}\left(E^{1}\right)$, we have $\langle\xi, \eta\rangle \in C_{0}\left(E^{0}\right)$.

Proof. For $\xi, \eta \in C_{d}\left(E^{1}\right)$, there exist sequences $\left\{\xi_{k}\right\}_{k \in \mathbb{N}},\left\{\eta_{l}\right\}_{l \in \mathbb{N}}$ in $C_{c}\left(E^{1}\right)$ such that $\lim _{k \rightarrow \infty}\left\|\xi-\xi_{k}\right\|=0$ and $\lim _{l \rightarrow \infty}\left\|\eta-\eta_{l}\right\|=0$ by Lemma 1.6. By Lemma 1.5] $\left\langle\xi_{k}, \eta_{l}\right\rangle \in C_{0}\left(E^{0}\right)$ for $k, l \in \mathbb{N}$. Since $\left\|\left\langle\xi_{k}, \eta\right\rangle-\left\langle\xi_{k}, \eta_{l}\right\rangle\right\| \leq\left\|\xi_{k}\right\| \cdot\left\|\eta-\eta_{l}\right\|$, we have $\left\langle\xi_{k}, \eta\right\rangle \in C_{0}\left(E^{0}\right)$ for each $k \in \mathbb{N}$. Since $\left\|\langle\xi, \eta\rangle-\left\langle\xi_{k}, \eta\right\rangle\right\| \leq\left\|\xi-\xi_{k}\right\| \cdot\|\eta\|$, we have $\langle\xi, \eta\rangle \in C_{0}\left(E^{0}\right)$.

By Lemma 1.7 we see that $C_{d}\left(E^{1}\right)$ is a linear space.

Lemma 1.8. The linear space $C_{d}\left(E^{1}\right)$ is a Banach space with respect to the norm $\|\cdot\|$.

Proof. It is easy to see that $\|\cdot\|$ satisfies the conditions for norms. Take a Cauchy sequence $\left\{\xi_{k}\right\}_{k \in \mathbb{N}}$ of $C_{d}\left(E^{1}\right)$. Since $\sup _{e \in E}\left|\xi^{\prime}(e)\right| \leq\left\|\xi^{\prime}\right\|$ for $\xi^{\prime} \in C_{d}\left(E^{1}\right)$, the sequence $\left\{\xi_{k}\right\}_{k \in \mathbb{N}}$ converges uniformly to some $\xi \in C\left(E^{1}\right)$. We will show that $\xi \in C_{d}\left(E^{1}\right)$ and $\left\{\xi_{k}\right\}_{k \in \mathbb{N}}$ converges to $\xi$ with respect to the norm $\|\cdot\|$. For any $\varepsilon>0$, there exists $K \in \mathbb{N}$ such that

$$
\sum_{e \in d^{-1}(v)}\left|\xi_{k}(e)-\xi_{l}(e)\right|^{2}<\varepsilon^{2},
$$

for all $k, l \geq K$ and all $v \in E^{0}$. Hence we have

$$
\sum_{e \in d^{-1}(v)}\left|\xi_{k}(e)-\xi(e)\right|^{2} \leq \varepsilon^{2},
$$

for all $k \geq K$ and all $v \in E^{0}$. This implies that $\left\|\xi_{k}-\xi\right\| \leq \varepsilon$ and $\|\left\langle\xi_{k}, \xi_{k}\right\rangle^{1 / 2}-$ $\langle\xi, \xi\rangle^{1 / 2} \| \leq \varepsilon$ for all $k \geq K$. Hence $\xi \in C_{d}\left(E^{1}\right)$ and $\left\{\xi_{k}\right\}_{k \in \mathbb{N}}$ converges to $\xi$ with respect to the norm $\|\cdot\|$. Thus we see that $C_{d}\left(E^{1}\right)$ is a Banach space. 
Note that we have $C_{c}\left(E^{1}\right) \subset C_{d}\left(E^{1}\right) \subset C_{0}\left(E^{1}\right)$ and that $C_{d}\left(E^{1}\right)$ is isomorphic to the completion of $C_{c}\left(E^{1}\right)$ with respect to the norm $\|\cdot\|$.

Lemma 1.9. For $\xi \in C_{d}\left(E^{1}\right)$ and $f \in C_{0}\left(E^{0}\right)$, we define $\xi f: E^{1} \rightarrow \mathbb{C}$ by $(\xi f)(e)=$ $\xi(e) f(d(e))$. Then $\xi f \in C_{d}\left(E^{1}\right)$ and $\langle\eta, \xi f\rangle=\langle\eta, \xi\rangle f$ for $\eta \in C_{d}\left(E^{1}\right)$.

Proof. It is easy to see that $\langle\xi f, \xi f\rangle=\bar{f}\langle\xi, \xi\rangle f$. Hence $\xi f \in C_{d}\left(E^{1}\right)$. The latter part is also easy.

Now we have proved the following.

Proposition 1.10. The Banach space $C_{d}\left(E^{1}\right)$ is a Hilbert $C_{0}\left(E^{0}\right)$-module under the operations in Lemma 1.7 and Lemma 1.9.

Before going further, we state a couple of lemmas on Hilbert modules arising from local homeomorphisms, which will be frequently used. Let $d$ be a local homeomorphism from $E^{1}$ to $E^{0}$, and $X^{0}$ be a closed subset of $E^{0}$. Set $X^{1}=d^{-1}\left(X^{0}\right)$ which is a closed subset of $E^{1}$. The restriction of $d$ to $X^{1}$ is a local homeomorphism to $X^{0}$. Hence we can define a Hilbert $C_{0}\left(X^{0}\right)$-module $C_{d}\left(X^{1}\right)$ as in Proposition 1.10. We use the notation $\|\cdot\|_{X}$ and $\langle\cdot, \cdot\rangle_{X}$ for denoting the norm and the inner product of $C_{d}\left(X^{1}\right)$.

Lemma 1.11. In the same notation as above, the natural map $C_{d}\left(E^{1}\right) \ni \xi \mapsto \dot{\xi} \in$ $C_{d}\left(X^{1}\right)$ defined by restriction is a surjective map. Moreover, for $\eta \in C_{d}\left(X^{1}\right)$, we can find $\xi \in C_{d}\left(E^{1}\right)$ with $\dot{\xi}=\eta$ and $\|\xi\|=\|\eta\|_{X}$.

Proof. It is easy to see that the restriction map $C_{d}\left(E^{1}\right) \ni \xi \mapsto \dot{\xi} \in C_{d}\left(X^{1}\right)$ is a well-defined norm-decreasing linear map. First we will show that for $\eta \in C_{d}\left(X^{1}\right)$, if there exists $\xi \in C_{d}\left(E^{1}\right)$ with $\dot{\xi}=\eta$, then we can find $\zeta \in C_{d}\left(E^{1}\right)$ with $\dot{\zeta}=\eta$ and $\|\zeta\|=\|\eta\|_{X}$. Take $\xi \in C_{d}\left(E^{1}\right)$ with $\dot{\xi}=\eta$. Set $L=\|\eta\|_{X}$ and define functions $f, g: E^{0} \rightarrow[0, \infty)$ by $f(v)=\min \left\{\langle\xi, \xi\rangle(v), L^{2}\right\}$ and $g(v)=L^{2} / \max \left\{L^{2},\langle\xi, \xi\rangle(v)\right\}$. Then we have $f \in C_{0}\left(E^{0}\right), 0 \leq f \leq L^{2}, g \in C\left(E^{0}\right), 0 \leq g \leq 1$ and $f=\langle\xi, \xi\rangle g$. We define $\zeta \in C\left(E^{1}\right)$ by $\zeta(e)=\xi(e) g(d(e))^{1 / 2}$. Then we have $\langle\zeta, \zeta\rangle=\langle\xi, \xi\rangle g=f$. Hence we see that $\zeta \in C_{d}\left(E^{1}\right)$ and $\|\zeta\| \leq L=\|\eta\|_{X}$. For $e \in X^{1}$ we have $g(d(e))=1$ since $\langle\xi, \xi\rangle(d(e))=\langle\eta, \eta\rangle_{X}(d(e)) \leq L^{2}$. Hence we have $\dot{\zeta}=\dot{\xi}=\eta$ and this implies that $\|\zeta\| \geq\|\eta\|_{X}$. Thus we have shown that $\zeta \in C_{d}\left(E^{1}\right)$ satisfies both $\dot{\zeta}=\eta$ and $\|\zeta\|=\|\eta\|_{X}$.

Next we show that the map $C_{d}\left(E^{1}\right) \ni \xi \mapsto \dot{\xi} \in C_{d}\left(X^{1}\right)$ is surjective. Take $\eta \in C_{d}\left(X^{1}\right)$ with $\eta \neq 0$. Set $L=\|\eta\|_{X}$. By Lemma 1.6, we can find $\eta_{1} \in C_{c}\left(X^{1}\right)$ such that $\left\|\eta-\eta_{1}\right\|_{X} \leq L / 2$ and $\left\|\eta_{1}\right\|_{X} \leq\|\eta\|_{X}=L$. Using Lemma 1.6 again, we can find $\eta_{2} \in C_{c}\left(X^{1}\right)$ such that

$$
\left\|\left(\eta-\eta_{1}\right)-\eta_{2}\right\|_{X} \leq \frac{L}{4}, \quad \text { and } \quad\left\|\eta_{2}\right\| \leq\left\|\eta-\eta_{1}\right\|_{X} \leq \frac{L}{2} .
$$

Recursively, we can find $\eta_{m} \in C_{c}\left(X^{1}\right)$ such that

$$
\left\|\eta-\sum_{k=1}^{m} \eta_{k}\right\|_{X} \leq \frac{L}{2^{m}}, \quad \text { and } \quad\left\|\eta_{m}\right\|_{X} \leq\left\|\eta-\sum_{k=1}^{m-1} \eta_{k}\right\|_{X} \leq \frac{L}{2^{m-1}} .
$$

Then we have $\eta=\sum_{k=1}^{\infty} \eta_{k}$. Since elements in $C_{c}\left(X^{1}\right)$ can be extended to elements in $C_{c}\left(E^{1}\right)$, we can find $\xi_{k} \in C_{d}\left(E^{1}\right)$ such that $\dot{\xi_{k}}=\eta_{k}$ and $\left\|\xi_{k}\right\|=\left\|\eta_{k}\right\|_{X} \leq L / 2^{k-1}$ by the former part of this proof. Since $C_{d}\left(E^{1}\right)$ is complete, we can define $\xi=$ $\sum_{k=1}^{\infty} \xi_{k} \in C_{d}\left(E^{1}\right)$. We have $\dot{\xi}=\sum_{k=1}^{\infty} \dot{\xi_{k}}=\eta$. Hence the map $C_{d}\left(E^{1}\right) \ni \xi \mapsto \dot{\xi} \in$ 
$C_{d}\left(X^{1}\right)$ is surjective. The last statement has been already proved in the argument above.

Since the restriction of $d$ to the open set $E^{1} \backslash X^{1}$ is a local homeomorphism to $E^{0} \backslash X^{0}$, we can define a Hilbert $C_{0}\left(E^{0} \backslash X^{0}\right)$-module $C_{d}\left(E^{1} \backslash X^{1}\right)$. The space $C_{d}\left(E^{1} \backslash X^{1}\right)$ is naturally considered as a subspace of $C_{d}\left(E^{1}\right)$,

$$
C_{d}\left(E^{1} \backslash X^{1}\right)=\left\{\xi \in C_{d}\left(E^{1}\right) \mid \xi(e)=0 \text { for } e \in X^{1}\right\} .
$$

Thus $C_{d}\left(E^{1} \backslash X^{1}\right)$ is a Hilbert $C_{0}\left(E^{0}\right)$-submodule of $C_{d}\left(E^{1}\right)$ and we see that

$$
\mathcal{K}\left(C_{d}\left(E^{1} \backslash X^{1}\right)\right)=\overline{\operatorname{span}}\left\{\theta_{\xi, \eta} \mid \xi, \eta \in C_{d}\left(E^{1} \backslash X^{1}\right)\right\} \subset \mathcal{K}\left(C_{d}\left(E^{1}\right)\right) .
$$

Note that we cannot consider $\mathcal{L}\left(C_{d}\left(E^{1} \backslash X^{1}\right)\right)$ as a subspace of $\mathcal{L}\left(C_{d}\left(E^{1}\right)\right)$ in general.

Lemma 1.12. For $\xi \in C_{d}\left(E^{1}\right)$, the following conditions are equivalent:

(i) $\xi \in C_{d}\left(E^{1} \backslash X^{1}\right)$,

(ii) $\dot{\xi}=0$,

(iii) $\langle\eta, \xi\rangle \in C_{0}\left(E^{0} \backslash X^{0}\right)$ for all $\eta \in C_{d}\left(E^{1}\right)$,

(iv) $\langle\xi, \xi\rangle \in C_{0}\left(E^{0} \backslash X^{0}\right)$,

(v) $\xi=\xi^{\prime} f$ for some $\xi^{\prime} \in C_{d}\left(E^{1}\right)$ and $f \in C_{0}\left(E^{0} \backslash X^{0}\right)$.

Proof. Clearly (i) is equivalent to (ii). (i) $\Rightarrow$ (iii) $\Rightarrow$ (iv) is obvious. For $\xi \in C_{d}\left(E^{1}\right)$ with $\langle\xi, \xi\rangle \in C_{0}\left(E^{0} \backslash X^{0}\right)$, we set $f=\langle\xi, \xi\rangle^{1 / 3}$ and

$$
\xi^{\prime}(e)= \begin{cases}\xi(e) f(d(e))^{-1} & \text { if } f(d(e)) \neq 0 \\ 0 & \text { if } f(d(e))=0 .\end{cases}
$$

Then we have $f \in C_{0}\left(E^{0} \backslash X^{0}\right), \xi^{\prime} \in C_{d}\left(E^{1}\right)$ and $\xi=\xi^{\prime} f$. This proves the implication (iv) $\Rightarrow(\mathrm{v})$. Finally it is easy to see that (i) follows from (v).

By (v) in Lemma 1.12, the submodule $C_{d}\left(E^{1} \backslash X^{1}\right)$ of $C_{d}\left(E^{1}\right)$ is closed under the action of $\mathcal{L}\left(C_{d}\left(E^{1}\right)\right)$. From this fact and Lemma 1.11, we can define a *homomorphism $\omega: \mathcal{L}\left(C_{d}\left(E^{1}\right)\right) \rightarrow \mathcal{L}\left(C_{d}\left(X^{1}\right)\right)$ by $\omega(a) \dot{\xi}=(\dot{a} \xi)$ for $a \in \mathcal{L}\left(C_{d}\left(E^{1}\right)\right)$ and $\xi \in C_{d}\left(E^{1}\right)$.

Lemma 1.13. For $a \in \mathcal{K}\left(C_{d}\left(E^{1}\right)\right)$, the following conditions are equivalent:

(i) $a \in \mathcal{K}\left(C_{d}\left(E^{1} \backslash X^{1}\right)\right)$,

(ii) $\omega(a)=0$,

(iii) $a \xi \in C_{d}\left(E^{1} \backslash X^{1}\right)$ for all $\xi \in C_{d}\left(E^{1}\right)$,

(iv) $\langle\eta, a \xi\rangle \in C_{0}\left(E^{0} \backslash X^{0}\right)$ for all $\xi, \eta \in C_{d}\left(E^{1}\right)$.

Proof. By the definition of $\omega$, we have (ii) $\Leftrightarrow$ (iii). By Lemma 1.12 (iii) $\Leftrightarrow$ (iv). Clearly (i) implies (iii). We will prove (iii) $\Rightarrow\left(\right.$ i). Take $a \in \mathcal{K}\left(C_{d}\left(E^{1}\right)\right)$ such that $a \xi \in C_{d}\left(E^{1} \backslash X^{1}\right)$ for all $\xi \in C_{d}\left(E^{1}\right)$. There exists an approximate unit $\left\{u_{i}\right\}_{i \in I}$ of $\mathcal{K}\left(C_{d}\left(E^{1}\right)\right)$ such that for each $i \in I, u_{i}$ is a finite linear sum of elements of the form $\theta_{\xi, \eta}$. Since we have $a=\lim a u_{i}$, to prove $a \in \mathcal{K}\left(C_{d}\left(E^{1} \backslash X^{1}\right)\right)$ it suffices to show that $a \theta_{\xi, \eta} \in \mathcal{K}\left(C_{d}\left(E^{1} \backslash X^{1}\right)\right)$ for arbitrary $\xi, \eta \in C_{d}\left(E^{1}\right)$. By the proof of Lemma 1.12, we can find $\xi^{\prime} \in C_{d}\left(E^{1}\right)$ and a positive element $f \in C_{0}\left(E^{0} \backslash X^{0}\right)$ such that $a \xi=\xi^{\prime} f$. Set $\xi^{\prime \prime}=\xi^{\prime} f^{1 / 2}$ and $\eta^{\prime \prime}=\eta f^{1 / 2}$. We have $\xi^{\prime \prime}, \eta^{\prime \prime} \in C_{d}\left(E^{1} \backslash X^{1}\right)$ and so

$$
a \theta_{\xi, \eta}=\theta_{a \xi, \eta}=\theta_{\xi^{\prime \prime}, \eta^{\prime \prime}} \in \mathcal{K}\left(C_{d}\left(E^{1} \backslash X^{1}\right)\right) .
$$

Thus we have $a \in \mathcal{K}\left(C_{d}\left(E^{1} \backslash X^{1}\right)\right)$.

Lemma 1.14. The restriction of $\omega$ to $\mathcal{K}\left(C_{d}\left(E^{1}\right)\right)$ is a surjective map to $\mathcal{K}\left(C_{d}\left(X^{1}\right)\right)$, whose kernel is $\mathcal{K}\left(C_{d}\left(E^{1} \backslash X^{1}\right)\right)$. 
Proof. The routine computation shows that $\omega\left(\theta_{\xi, \eta}\right)=\theta_{\dot{\xi}, \dot{\eta}}$ for $\xi, \eta \in C_{d}\left(E^{1}\right)$. Hence by Lemma 1.11, the restriction of $\omega$ to $\mathcal{K}\left(C_{d}\left(E^{1}\right)\right)$ is a surjective map onto $\mathcal{K}\left(C_{d}\left(X^{1}\right)\right)$. We have $\mathcal{K}\left(C_{d}\left(E^{1}\right)\right) \cap \operatorname{ker} \omega=\mathcal{K}\left(C_{d}\left(E^{1} \backslash X^{1}\right)\right)$ by Lemma 1.13.

There exists a $*$-homomorphism $\pi: C_{b}\left(E^{1}\right) \rightarrow \mathcal{L}\left(C_{d}\left(E^{1}\right)\right)$ defined by $(\pi(f) \xi)(e)$ $=f(e) \xi(e)$ for $f \in C_{b}\left(E^{1}\right)$ and $\xi \in C_{d}\left(E^{1}\right)$. Note that $\pi$ is injective. We will show that for $f \in C_{b}\left(E^{1}\right), \pi(f) \in \mathcal{K}\left(C_{d}\left(E^{1}\right)\right)$ if and only if $f \in C_{0}\left(E^{1}\right)$.

Lemma 1.15. If $f \in C_{b}\left(E^{1}\right)$ and $\xi_{k}, \eta_{k} \in C_{d}\left(E^{1}\right)$ for $k=1, \ldots, m$ satisfy $f=$ $\sum_{k=1}^{m} \xi_{k} \overline{\eta_{k}}$ and $\xi_{k}(e) \overline{\eta_{k}\left(e^{\prime}\right)}=0$ for any $k$ and any $e, e^{\prime} \in E^{1}$ with $e \neq e^{\prime}, d(e)=$ $d\left(e^{\prime}\right)$, then we have $\pi(f)=\sum_{k=1}^{m} \theta_{\xi_{k}, \eta_{k}}$.

Proof. For $\zeta \in C_{d}\left(E^{1}\right)$ and $e \in E^{1}$, we have

$$
\begin{aligned}
\left(\left(\sum_{k=1}^{m} \theta_{\xi_{k}, \eta_{k}}\right) \zeta\right)(e) & =\sum_{k=1}^{m}\left(\xi_{k}\left\langle\eta_{k}, \zeta\right\rangle\right)(e)=\sum_{k=1}^{m}\left(\xi_{k}(e) \sum_{e^{\prime} \in d^{-1}(d(e))} \overline{\left.\eta_{k}\left(e^{\prime}\right) \zeta\left(e^{\prime}\right)\right)}\right. \\
& =\sum_{k=1}^{m} \sum_{e^{\prime} \in d^{-1}(d(e))}\left(\xi_{k}(e) \overline{\eta_{k}\left(e^{\prime}\right)} \zeta\left(e^{\prime}\right)\right)=\sum_{k=1}^{m}\left(\xi_{k}(e) \overline{\eta_{k}(e)} \zeta(e)\right) \\
& =f(e) \zeta(e)=(\pi(f) \zeta)(e) .
\end{aligned}
$$

Thus we have $\pi(f)=\sum_{k=1}^{m} \theta_{\xi_{k}, \eta_{k}}$.

Lemma 1.16. For $f \in C_{c}\left(E^{1}\right)$, we can find $\xi_{k}, \eta_{k} \in C_{c}\left(E^{1}\right)$ for $k=1, \ldots, m$ such that $f=\sum_{k=1}^{m} \xi_{k} \overline{\eta_{k}}$ and $\xi_{k}(e) \overline{\eta_{k}\left(e^{\prime}\right)}=0$ for any $k$ and any $e, e^{\prime} \in E^{1}$ with $e \neq e^{\prime}$ and $d(e)=d\left(e^{\prime}\right)$.

Proof. We denote the support of $f$ by $X=\operatorname{supp}(f)$, which is a compact subset of $E^{1}$. Since $d$ is a local homeomorphism, for each $e \in X$ there exists an open and relatively compact neighborhood $U_{e}$ of $e$ such that the restriction of $d$ to $U_{e}$ is injective. Since $X$ is compact, we can find $e_{1}, \ldots, e_{m} \in X$ such that $X \subset \bigcup_{k=1}^{m} U_{e_{k}}$. Take $\zeta_{1}, \ldots, \zeta_{m} \in C_{c}\left(E^{1}\right)$ satisfying $0 \leq \zeta_{k} \leq 1, \operatorname{supp}\left(\zeta_{k}\right) \subset U_{e_{k}}$ for each $k$, and $\sum_{k=1}^{m} \zeta_{k}(e)=1$ for all $e \in X$. For each $k$, we define $\xi_{k}=f \zeta_{k}^{1 / 2}$ and $\eta_{k}=\zeta_{k}^{1 / 2}$. Then we have

$$
\sum_{k=1}^{m} \xi_{k} \overline{\eta_{k}}=f \sum_{k=1}^{m} \zeta_{k}=f
$$

For $k=1,2, \ldots, m$, we have $\xi_{k}(e) \overline{\eta_{k}\left(e^{\prime}\right)}=0$ for $e, e^{\prime} \in E^{1}$ with $e \neq e^{\prime}$ and $d(e)=$ $d\left(e^{\prime}\right)$ because $\operatorname{supp}\left(\xi_{k}\right), \operatorname{supp}\left(\eta_{k}\right) \subset U_{e_{k}}$ and the restriction of $d$ to $U_{e_{k}}$ is injective. We are done.

Proposition 1.17. For $f \in C_{b}\left(E^{1}\right)$, we have $\pi(f) \in \mathcal{K}\left(C_{d}\left(E^{1}\right)\right)$ if and only if $f \in C_{0}\left(E^{1}\right)$.

Proof. For $f \in C_{c}\left(E^{1}\right)$, we have $\pi(f) \in \mathcal{K}\left(C_{d}\left(E^{1}\right)\right)$ by Lemma 1.15 and Lemma 1.16. Hence we have $\pi(f) \in \mathcal{K}\left(C_{d}\left(E^{1}\right)\right)$ for every $f \in C_{0}\left(E^{1}\right)$. Conversely take $f \notin C_{0}\left(E^{1}\right)$. Then there exists $\varepsilon>0$ such that the closed set

$$
U=\left\{e \in E^{1}|| f(e) \mid \geq \varepsilon\right\}
$$

is not compact. Take $\xi_{1}, \ldots, \xi_{m}, \eta_{1}, \ldots, \eta_{m} \in C_{c}\left(E^{1}\right)$ arbitrarily, and we will show that $\left\|\pi(f)-\sum_{k=1}^{m} \theta_{\xi_{k}, \eta_{k}}\right\| \geq \varepsilon$. Since the closed set $U$ is not compact, we can find $e_{0} \in U$ such that $e_{0} \notin \operatorname{supp}\left(\eta_{k}\right)$ for every $k=1, \ldots, m$. Take an open neighborhood $U_{0} \subset E^{1}$ of $e_{0}$ such that the restriction of $d$ to $U_{0}$ is injective and $U_{0} \cap \operatorname{supp}\left(\eta_{k}\right)=\emptyset$ 
for every $k=1, \ldots, m$. Set $\zeta \in C_{c}\left(U_{0}\right) \subset C_{d}\left(E^{1}\right)$ with $0 \leq \zeta \leq 1$ and $\zeta\left(e_{0}\right)=1$. We have

$$
\|\zeta\|=\sup _{v \in E^{0}}\left(\sum_{e \in d^{-1}(v)}|\zeta(e)|^{2}\right)^{1 / 2}=\sup _{e \in U_{0}}|\zeta(e)|=1,
$$

and

$$
\begin{aligned}
\left\|\left(\pi(f)-\sum_{k=1}^{m} \theta_{\xi_{k}, \eta_{k}}\right) \zeta\right\| & =\|\pi(f) \zeta\|=\sup _{v \in E^{0}}\left(\sum_{e \in d^{-1}(v)}|f(e) \zeta(e)|^{2}\right)^{1 / 2} \\
& =\sup _{e \in U}|f(e) \zeta(e)| \geq \varepsilon .
\end{aligned}
$$

Hence we get

$$
\left\|\pi(f)-\sum_{k=1}^{m} \theta_{\xi_{k}, \eta_{k}}\right\| \geq \varepsilon .
$$

Since $C_{c}\left(E^{1}\right)$ is dense in $C_{d}\left(E^{1}\right)$, we have $\mathcal{K}\left(C_{d}\left(E^{1}\right)\right)=\overline{\operatorname{span}}\left\{\theta_{\xi, \eta} \mid \xi, \eta \in C_{c}\left(E^{1}\right)\right\}$. Hence we get $\pi(f) \notin \mathcal{K}\left(C_{d}\left(E^{1}\right)\right)$.

Remark 1.18. We can show that $\mathcal{K}\left(C_{d}\left(E^{1}\right)\right)$ is a continuous trace $C^{*}$-algebra over the open subset $d\left(E^{1}\right)$ of $E^{0}$. For each $v \in E^{0}$, there exists a $*$-homomorphism $\mathcal{K}\left(C_{d}\left(E^{1}\right)\right) \rightarrow \mathcal{K}\left(H_{v}\right)$ where $H_{v}$ is a Hilbert space whose dimension is the cardinality of $d^{-1}(v)$ (when $v \notin d\left(E^{1}\right)$, we set $H_{v}=0$ ). Hence elements of $\mathcal{K}\left(C_{d}\left(E^{1}\right)\right.$ ) can be considered as "compact operator valued" continuous functions on $E^{0}$ which vanish at infinity. Similarly elements of $\mathcal{L}\left(C_{d}\left(E^{1}\right)\right)$ can be considered as "bounded operator valued" bounded continuous functions on $E^{0}$.

Each $f \in C_{b}\left(E^{1}\right)$ defines a topological correspondence $\left(E^{1}, d, f\right)$ from $E^{0}$ to $\mathbb{C}$. The element $f \in C_{b}\left(E^{1}\right)$ also defines $\pi(f) \in \mathcal{L}\left(C_{d}\left(E^{1}\right)\right)$ which can be considered as a "diagonal operator valued" continuous function on $E^{0}$. Thus topological correspondences from $E^{0}$ to $\mathbb{C}$ can be identified with "diagonal operator valued" continuous functions on $E^{0}$.

So far, we only used the data of "domain" $\left(E^{1}, d\right)$ of the topological correspondence $\left(E^{1}, d, r\right)$. Now we will use the continuous map $r: E^{1} \rightarrow F^{0}$ to define a left action $\pi_{r}$ of $C_{0}\left(F^{0}\right)$ on the Hilbert $C_{0}\left(E^{0}\right)$-module $C_{d}\left(E^{1}\right)$. The continuous map $r: E^{1} \rightarrow F^{0}$ gives us a $*$-homomorphism $C_{0}\left(F^{0}\right) \ni f \rightarrow f \circ r \in C_{b}\left(E^{1}\right)$. Denote the composition of this map and $\pi: C_{b}\left(E^{1}\right) \rightarrow \mathcal{L}\left(C_{d}\left(E^{1}\right)\right)$ by $\pi_{r}: C_{0}\left(F^{0}\right) \rightarrow \mathcal{L}\left(C_{d}\left(E^{1}\right)\right)$. Explicitly, $\left(\pi_{r}(f) \xi\right)(e)=f(r(e)) \xi(e)$ for $e \in E^{1}, f \in C_{0}\left(F^{0}\right)$ and $\xi \in C_{d}\left(E^{1}\right)$. In this way, we get a $C^{*}$-correspondence $C_{d}\left(E^{1}\right)$ from $C_{0}\left(F^{0}\right)$ to $C_{0}\left(E^{0}\right)$ by using a topological correspondence $\left(E^{1}, d, r\right)$ from $E^{0}$ to $F^{0}$.

Remark 1.19. For $f \in C_{0}\left(F^{0}\right)$, we can identify a "diagonal operator valued" continuous function $\pi_{r}(f)$ with a topological correspondence $\left(E^{1}, d, f \circ r\right)$ from $E^{0}$ to $\mathbb{C}$ (see Remark 1.18). Thus, the map $\pi_{r}: C_{0}\left(F^{0}\right) \rightarrow \mathcal{L}\left(C_{d}\left(E^{1}\right)\right)$ is given by just composing a topological correspondence $\left(E^{1}, d, r\right)$. This observation is useful when we compute the $K$-groups of $\mathcal{O}(E)$, and will be further studied in Section 2 for two special examples.

Lemma 1.20. The left action $\pi_{r}: C_{0}\left(F^{0}\right) \rightarrow \mathcal{L}\left(C_{d}\left(E^{1}\right)\right)$ is non-degenerate.

Proof. Take $\xi \in C_{c}\left(E^{1}\right)$, and set $K=\operatorname{supp}(\xi)$ which is a compact subset of $E^{1}$. Since $r(K)$ is compact in $F^{0}$, we can find $f \in C_{0}\left(F^{0}\right)$ such that $f(v)=1$ for all 
$v \in r(K)$. Then we have $\pi_{r}(f) \xi=\xi$. Since $C_{c}\left(E^{1}\right)$ is dense in $C_{d}\left(E^{1}\right)$, we see that

$$
\left\{\pi_{r}(f) \xi \in C_{d}\left(E^{1}\right) \mid f \in C_{0}\left(F^{0}\right), \xi \in C_{d}\left(E^{1}\right)\right\}
$$

is dense in $C_{d}\left(E^{1}\right)$. We are done.

We define two open subsets $F_{\text {sce }}^{0}, F_{\text {fin }}^{0}$ of $F^{0}$ by

$$
\begin{aligned}
& F_{\text {sce }}^{0}=\left\{v \in F^{0} \mid v \text { has a neighborhood } V \text { such that } r^{-1}(V)=\emptyset\right\}, \\
& F_{\text {fin }}^{0}=\left\{v \in F^{0} \mid v \text { has a neighborhood } V \text { such that } r^{-1}(V) \text { is compact }\right\} .
\end{aligned}
$$

We will justify the notation in Section 2 Obviously $F_{\text {sce }}^{0} \subset F_{\text {fin }}^{0}$ and $F_{\text {sce }}^{0}=$ $F^{0} \backslash \overline{r\left(E^{1}\right)}$. Since $F_{\text {sce }}^{0}, F_{\text {fin }}^{0}$ are open, we can consider $C_{0}\left(F_{\text {sce }}^{0}\right)$ and $C_{0}\left(F_{\text {fin }}^{0}\right)$ as ideals of $C_{0}\left(F^{0}\right)$.

Lemma 1.21. Let $v \in F_{\text {fin }}^{0}$ and $U$ be an open subset of $E^{1}$ with $r^{-1}(v) \subset U$. Then there exists a neighborhood $V$ of $v$ such that $r^{-1}(V) \subset U$.

Proof. Since $v \in F_{\text {fin }}^{0}$, there exists a neighborhood $V_{1}$ of $v$ such that $r^{-1}\left(V_{1}\right)$ is compact. To derive a contradiction, suppose that for all neighborhoods $V$ of $v$ with $V \subset V_{1}$, we can find $e_{V} \in r^{-1}(V)$ with $e_{V} \notin U$. Since the net $\left\{e_{V}\right\}$ is in the compact set $r^{-1}\left(V_{1}\right)$, we can find a subnet $\left\{e_{V_{\lambda}}\right\}$ of $\left\{e_{V}\right\}$ such that $e_{V_{\lambda}}$ converges to some $e \in r^{-1}\left(V_{1}\right)$. Since $U$ is open, we have $e \notin U$. By the continuity of $r$, we have $r(e)=v$. This contradicts the fact that $r^{-1}(v) \subset U$. Hence we can find a neighborhood $V$ of $v$ such that $r^{-1}(V) \subset U$.

Lemma 1.22. For $v \in F_{\text {fin }}^{0} \backslash F_{\text {sce }}^{0}$ we have $r^{-1}(v) \neq \emptyset$.

Proof. If $v \in F_{\text {fin }}^{0}$ satisfies $r^{-1}(v)=\emptyset$, then there exists a neighborhood $V$ of $v$ such that $r^{-1}(V)=\emptyset$ by Lemma 1.21. Thus we have $v \in F_{\text {sce. }}^{0}$.

Lemma 1.23. For a compact set $X \subset F_{\text {fin }}^{0}$, the subset $r^{-1}(X)$ of $E^{1}$ is compact.

Proof. For each $v \in X$, there exists a neighborhood $V_{v}$ of $v$ such that $r^{-1}\left(V_{v}\right)$ is compact. Since $X$ is compact, we can find $v_{1}, \ldots, v_{n} \in X$ with $X \subset \bigcup_{i=1}^{n} V_{v_{i}}$. Since $r^{-1}(X) \subset \bigcup_{i=1}^{n} r^{-1}\left(V_{v_{i}}\right)$, the set $r^{-1}(X)$ is compact.

Proposition 1.24. We have $\operatorname{ker} \pi_{r}=C_{0}\left(F_{\mathrm{sce}}^{0}\right)$ and $\pi_{r}^{-1}\left(\mathcal{K}\left(C_{d}\left(E^{1}\right)\right)\right)=C_{0}\left(F_{\text {fin }}^{0}\right)$.

Proof. We have ker $\pi_{r}=C_{0}\left(F_{\text {sce }}^{0}\right)$ because

$$
\begin{aligned}
f \in \operatorname{ker} \pi_{r} & \Longleftrightarrow f(r(e))=0 \text { for all } e \in E^{1} \\
& \Longleftrightarrow f(v)=0 \text { for all } v \in \overline{r\left(E^{1}\right)} \\
& \Longleftrightarrow f \in C_{0}\left(F_{\mathrm{sce}}^{0}\right) .
\end{aligned}
$$

To prove the latter, it suffices to show that for $f \in C_{0}\left(F^{0}\right), f \circ r \in C_{0}\left(E^{1}\right)$ if and only if $f \in C_{0}\left(F_{\text {fin }}^{0}\right)$ by Proposition 1.17. If $f \in C_{0}\left(F_{\text {fin }}^{0}\right)$, we have

$$
\left\{e \in E^{1}|| f(r(e)) \mid \geq \varepsilon\right\}=r^{-1}\left(\left\{v \in E^{0}|| f(v) \mid \geq \varepsilon\right\}\right)
$$

for any $\varepsilon>0$. Since $\left\{v \in E^{0}|| f(v) \mid \geq \varepsilon\right\}$ is a compact subset of $F_{\text {fin }}^{0}$, Lemma 1.23 shows that $\left\{e \in E^{1}|| f(r(e)) \mid \geq \varepsilon\right\}$ is compact. Hence $f \circ r \in C_{0}\left(E^{1}\right)$. Now suppose $f \notin C_{0}\left(F_{\text {fin }}^{0}\right)$. There exists $v_{0} \notin F_{\text {fin }}^{0}$ such that $\left|f\left(v_{0}\right)\right|>0$. Take $\varepsilon>0$ with $\varepsilon<\left|f\left(v_{0}\right)\right|$ and set $V=\left\{v \in E^{0}|| f(v) \mid \geq \varepsilon\right\}$. Then $V$ is a neighborhood of $v_{0}$. Since $v_{0} \notin F_{\text {fin }}^{0}, r^{-1}(V)$ is not compact. Since $\left\{e \in E^{1}|| f(r(e)) \mid \geq \varepsilon\right\}=r^{-1}(V)$, we have $f \circ r \notin C_{0}\left(E^{1}\right)$. Therefore we have $C_{0}\left(F_{\text {fin }}^{0}\right)=\pi_{r}^{-1}\left(\mathcal{K}\left(C_{d}\left(E^{1}\right)\right)\right)$. 
Finally we define a composition of two topological correspondences and prove that this relates to the interior tensor products of $C^{*}$-correspondences. Let $E^{0}, F^{0}$, $G^{0}$ be locally compact spaces, and $\left(E^{1}, d, r\right),\left(F^{1}, d^{\prime}, r^{\prime}\right)$ be topological correspondences from $E^{0}$ to $F^{0}$ and $F^{0}$ to $G^{0}$ respectively. Namely, $d: E^{1} \rightarrow E^{0}, d^{\prime}: F^{1} \rightarrow$ $F^{0}$ are local homeomorphisms and $r: E^{1} \rightarrow F^{0}, r^{\prime}: F^{1} \rightarrow G^{0}$ are continuous maps. We define

$$
E^{2}=\left\{\left(e^{\prime}, e\right) \in F^{1} \times E^{1} \mid d^{\prime}\left(e^{\prime}\right)=r(e)\right\},
$$

which is a closed subset of $F^{1} \times E^{1}$. We define a map $d^{\prime \prime}: E^{2} \rightarrow E^{0}$ and $r^{\prime \prime}: E^{2} \rightarrow G^{0}$ by $d^{\prime \prime}\left(\left(e^{\prime}, e\right)\right)=d(e)$ and $r^{\prime \prime}\left(\left(e^{\prime}, e\right)\right)=r^{\prime}\left(e^{\prime}\right)$ for $\left(e^{\prime}, e\right) \in E^{2}$.

Lemma 1.25. The triple $\left(E^{2}, d^{\prime \prime}, r^{\prime \prime}\right)$ is a topological correspondence from $E^{0}$ to $G^{0}$.

Proof. Since $E^{2}$ is a closed subset of $F^{1} \times E^{1}$, it is a locally compact space. Clearly $r^{\prime \prime}: E^{2} \rightarrow G^{0}$ is continuous. We only need to show that $d^{\prime \prime}: E^{2} \rightarrow E^{0}$ is locally homeomorphic. Take $\left(e^{\prime}, e\right) \in E^{2}$. There exists an open neighborhood $U^{\prime}$ of $e^{\prime} \in F^{1}$ such that the restriction of $d^{\prime}$ to $U^{\prime}$ is a homeomorphism onto $d^{\prime}\left(U^{\prime}\right)$, and that $d^{\prime}\left(U^{\prime}\right)$ is an open subset of $F^{0}$. We can find an open neighborhood $U$ of $e \in E^{1}$ with $U \subset r^{-1}\left(d^{\prime}\left(U^{\prime}\right)\right)$ such that the restriction of $d$ to $U$ is a homeomorphism onto $d(U)$, and that $d(U)$ is open. Set $U^{\prime \prime}=E^{2} \cap\left(U^{\prime} \times U\right)$ which is an open neighborhood of $\left(e^{\prime}, e\right) \in E^{2}$. For $v \in d(U)$, there exist a unique element $e_{v} \in U$ satisfying $d\left(e_{v}\right)=v$ and a unique element $e_{v}^{\prime} \in U^{\prime}$ satisfying $d^{\prime}\left(e_{v}^{\prime}\right)=r\left(e_{v}\right)$. The map $d(U) \ni v \mapsto\left(e_{v}^{\prime}, e_{v}\right) \in U^{\prime \prime}$ is a continuous map which is the inverse of the restriction of $d^{\prime \prime}$ to $U^{\prime \prime}$. Hence the restriction of $d^{\prime \prime}$ to $U^{\prime \prime}$ is a homeomorphism onto $d(U)$ which is open. Therefore $d^{\prime \prime}: E^{2} \rightarrow E^{0}$ is a local homeomorphism.

The topological correspondence $\left(E^{2}, d^{\prime \prime}, r^{\prime \prime}\right)$ defined above is called the composition of two topological correspondences $\left(E^{1}, d, r\right)$ and $\left(F^{1}, d^{\prime}, r^{\prime}\right)$. This composition clearly satisfies associativity. When $F^{1}=F^{0}$ and $d^{\prime}=\mathrm{id}$, the composition of two topological correspondences $\left(E^{1}, d, r\right)$ and $\left(F^{0}, \mathrm{id}, r^{\prime}\right)$ is $\left(E^{1}, d, r^{\prime} \circ r\right)$. We will show that the compositions of topological correspondences correspond to the interior tensor products of $C^{*}$-correspondences. We need one lemma.

Lemma 1.26. Let $d$ be a local homeomorphism from $E^{1}$ to $E^{0}$. Suppose that a subset $X$ of $C_{c}\left(E^{1}\right)$ has the property that for every open subset $U$ of $E^{1}$, every element of $C_{c}(U)$ can be uniformly approximated by elements of $X \cap C_{c}(U)$. Then $X$ is dense in $C_{d}\left(E^{1}\right)$ with respect to the norm $\|\cdot\|$

Proof. Let $U$ be an open subset of $E^{1}$ such that the restriction of $d$ to $U$ is injective. Then, we have $\|\xi\|=\sup _{e \in U}|\xi(e)|$ for $\xi \in C_{c}(U) \subset C_{c}\left(E^{1}\right)$. Hence we see that elements in $C_{c}(U)$ can be approximated by elements in $X \cap C_{c}(U)$ with respect to the norm $\|\cdot\|$. By using a partition of unity, we can show that an arbitrary element in $C_{c}\left(E^{1}\right)$ is a finite sum of continuous functions whose supports are compact sets on which $d$ is injective. Hence $X$ is dense in $C_{c}\left(E^{1}\right)$ with respect to the norm $\|\cdot\|$. This completes the proof because $C_{c}\left(E^{1}\right)$ is dense in $C_{d}\left(E^{1}\right)$ with respect to the norm $\|\cdot\|$ by Lemma 1.6.

Proposition 1.27. We have

$$
C_{d^{\prime \prime}}\left(E^{2}\right) \cong C_{d^{\prime}}\left(F^{1}\right) \otimes C_{d}\left(E^{1}\right)
$$

as $C^{*}$-correspondences from $C_{0}\left(G^{0}\right)$ to $C_{0}\left(E^{0}\right)$. 
Proof. There exists a linear map $\Psi: C_{c}\left(F^{1}\right) \odot C_{c}\left(E^{1}\right) \rightarrow C_{c}\left(E^{2}\right)$ defined by

$$
\Psi(\xi \otimes \eta)\left(e^{\prime}, e\right)=\xi\left(e^{\prime}\right) \eta(e) \quad \text { for }\left(e^{\prime}, e\right) \in E^{2} .
$$

Since $d^{\prime}\left(e^{\prime}\right)=r(e)$ for $\left(e^{\prime}, e\right) \in E^{2}$, we have

$$
\Psi((\xi f) \otimes \eta)=\Psi\left(\xi \otimes\left(\pi_{r}(f) \eta\right)\right)
$$

for $\xi \in C_{c}\left(F^{1}\right), \eta \in C_{c}\left(E^{1}\right)$ and $f \in C_{0}\left(F^{0}\right)$. Hence $\Psi$ factors through the map $\Psi^{\prime}: C_{c}\left(F^{1}\right) \odot_{C_{0}\left(F^{0}\right)} C_{c}\left(E^{1}\right) \rightarrow C_{c}\left(E^{2}\right)$. Routine computation shows that $\langle x, y\rangle=\left\langle\Psi^{\prime}(x), \Psi^{\prime}(y)\right\rangle$ for $x, y \in C_{c}\left(F^{1}\right) \odot_{C_{0}\left(F^{0}\right)} C_{c}\left(E^{1}\right)$. Hence $\Psi^{\prime}$ extends to the isometric linear map $\Psi^{\prime \prime}: C_{d^{\prime}}\left(F^{1}\right) \otimes C_{d}\left(E^{1}\right) \rightarrow C_{d^{\prime \prime}}\left(E^{2}\right)$, which is easily shown to be a bimodule map. To prove that $\Psi^{\prime \prime}$ is surjective, it suffices to show that the image of $\Psi^{\prime}$ is dense in $C_{d^{\prime \prime}}\left(E^{2}\right)$ because $\Psi^{\prime \prime}$ is isometric. It is well known that for each open subset $U$ of $E^{2}$, the intersection of the image of $\Psi$ and $C_{c}(U)$ is dense in $C_{c}(U) \subset C_{c}\left(E^{2}\right)$ with respect to the sup norm. By Lemma 1.26, the image of $\Psi^{\prime}$ is dense in $C_{d^{\prime \prime}}\left(E^{2}\right)$ with respect to the norm $\|\cdot\|$. Hence $\Psi^{\prime \prime}$ is surjective. Thus $C_{d^{\prime}}\left(F^{1}\right) \otimes C_{d}\left(E^{1}\right)$ is isomorphic to $C_{d^{\prime \prime}}\left(E^{2}\right)$ via $\Psi^{\prime \prime}$.

\section{2. $C^{*}$-ALGEBRAS ARISING FROM TOPOLOGICAL GRAPHS}

In this section, we introduce a notion of topological graphs and give a method to define $C^{*}$-algebras from them. This construction is a generalization of ones of both graph algebras and homeomorphism $C^{*}$-algebras.

Definition 2.1. A topological graph is a quadruple $E=\left(E^{0}, E^{1}, d, r\right)$ where $E^{0}, E^{1}$ are locally compact spaces, $d: E^{1} \rightarrow E^{0}$ is a local homeomorphism and $r: E^{1} \rightarrow E^{0}$ is a continuous map.

Note that $d, r: E^{1} \rightarrow E^{0}$ are not necessarily surjective nor injective. We think that $E^{0}$ is a set of vertices and $E^{1}$ is a set of edges and that an edge $e \in E^{1}$ is directed from its domain $d(e) \in E^{0}$ to its range $r(e) \in E^{0}$. When $E^{0}$ is a discrete set, then $E^{1}$ is also discrete. In this case, we call $E=\left(E^{0}, E^{1}, d, r\right)$ a discrete graph.

For a topological graph $E=\left(E^{0}, E^{1}, d, r\right)$, the triple $\left(E^{1}, d, r\right)$ is a topological correspondence from $E^{0}$ to itself. Hence we can consider a quadruple $E=$ $\left(E^{0}, E^{1}, d, r\right)$ as a kind of dynamical systems. This point of view is very important and we extend many notions and results from ordinary dynamical systems defined by homeomorphisms to our setting (see Section [5] in this paper or [Ka3]).

Take a topological graph $E=\left(E^{0}, E^{1}, d, r\right)$. For $n=2,3, \ldots$, we define a space $E^{n}$ of paths with length $n$ by

$$
E^{n}=\left\{\left(e_{n}, \ldots, e_{2}, e_{1}\right) \in E^{1} \times \cdots \times E^{1} \times E^{1} \mid d\left(e_{k+1}\right)=r\left(e_{k}\right)(1 \leq k \leq n-1)\right\} .
$$

We define domain and range maps $d^{n}, r^{n}: E^{n} \rightarrow E^{0}$ by $d^{n}(e)=d\left(e_{1}\right)$ and $r^{n}(e)=$ $r\left(e_{n}\right)$ for $e=\left(e_{n}, \ldots, e_{1}\right) \in E^{n}$. We write $d^{1}=d$ and $r^{1}=r$. We sometimes consider $E^{0}$ as the set of paths with length 0 . The domain and range maps $d^{0}, r^{0}: E^{0} \rightarrow E^{0}$ are defined by $d^{0}=r^{0}=$ id. Note that the order how to denote paths is the same as the one of composition of maps.

$$
\left(e_{n}, e_{n-1}, \ldots, e_{2}, e_{1}\right) \in E^{n} \leadsto r\left(e_{n}\right) e_{n} \cdot \stackrel{e_{n-1}}{\longleftarrow} \cdots \stackrel{e_{2}}{\longleftarrow} e_{1} d\left(e_{1}\right)
$$

For $n=2,3, \ldots$, the triple $\left(E^{n}, d^{n}, r^{n}\right)$ is nothing but the $n$-times composition of the topological correspondence $\left(E^{1}, d, r\right)$. Hence by Lemma 1.25, $E^{n}$ is a locally compact space, $d^{n}$ is a local homeomorphism and $r^{n}$ is a continuous map for each $n \in \mathbb{N}$. From the topological correspondence $\left(E^{1}, d, r\right)$, we get a $C^{*}$-correspondence 
$C_{d}\left(E^{1}\right)$ over $C_{0}\left(E^{0}\right)$ whose left action is denoted by $\pi_{r}: C_{0}\left(E^{0}\right) \rightarrow \mathcal{L}\left(C_{d}\left(E^{1}\right)\right)$ as in Section 10 The $C^{*}$-correspondences $C_{d^{n}}\left(E^{n}\right)$ defined by the topological correspondence $\left(E^{n}, d^{n}, r^{n}\right)$ have the property that for any $n, m \in \mathbb{N}$

$$
C_{d^{n+m}}\left(E^{n+m}\right) \cong C_{d^{n}}\left(E^{n}\right) \otimes C_{d^{m}}\left(E^{m}\right) \quad\left(\text { as } C^{*} \text {-correspondences over } C_{0}\left(E^{0}\right)\right)
$$

by Proposition 1.27 and for $n \geq 1$

$$
C_{d^{n}}\left(E^{n}\right)=\overline{\operatorname{span}}\left\{\xi_{n} \otimes \cdots \otimes \xi_{2} \otimes \xi_{1} \mid \xi_{i} \in C_{d^{1}}\left(E^{1}\right)\right\} .
$$

Note that the $C^{*}$-correspondence $C_{d^{0}}\left(E^{0}\right)$ coincides with $C_{0}\left(E^{0}\right)$, and left and right actions are just multiplication. As long as no confusion arises, we omit the superscript $n$ and simply write $d, r$ for $d^{n}, r^{n}$.

Definition 2.2. Let $E=\left(E^{0}, E^{1}, d, r\right)$ be a topological graph. A Toeplitz E-pair on a $C^{*}$-algebra $A$ is a pair of maps $T=\left(T^{0}, T^{1}\right)$ where $T^{0}: C_{0}\left(E^{0}\right) \rightarrow A$ is a *-homomorphism and $T^{1}: C_{d}\left(E^{1}\right) \rightarrow A$ is a linear map satisfying

(i) $T^{1}(\xi)^{*} T^{1}(\eta)=T^{0}(\langle\xi, \eta\rangle)$ for $\xi, \eta \in C_{d}\left(E^{1}\right)$,

(ii) $T^{0}(f) T^{1}(\xi)=T^{1}\left(\pi_{r}(f) \xi\right)$ for $f \in C_{0}\left(E^{0}\right)$ and $\xi \in C_{d}\left(E^{1}\right)$.

We denote by $\mathcal{T}(E)$ the universal $C^{*}$-algebra generated by a Toeplitz $E$-pair.

For a Toeplitz $E$-pair $T=\left(T^{0}, T^{1}\right)$, we see that $\left\|T^{0}(f)\right\| \leq\|f\|$ and $\left\|T^{1}(\xi)\right\| \leq$ $\|\xi\|$ because $T^{0}$ is a $*$-homomorphism and

$$
\left\|T^{1}(\xi)\right\|^{2}=\left\|T^{1}(\xi)^{*} T^{1}(\xi)\right\|=\left\|T^{0}(\langle\xi, \xi\rangle)\right\| \leq\|\langle\xi, \xi\rangle\|=\|\xi\|^{2} .
$$

Hence the universal $C^{*}$-algebra $\mathcal{T}(E)$ generated by a Toeplitz $E$-pair exists (see Section 3 for a concrete construction of $\mathcal{T}(E))$. We have $T^{1}(\xi) T^{0}(f)=T^{1}(\xi f)$ for $f \in C_{0}\left(E^{0}\right)$ and $\xi \in C_{d}\left(E^{1}\right)$ because

$$
\left(T^{1}(\xi) T^{0}(f)-T^{1}(\xi f)\right)^{*}\left(T^{1}(\xi) T^{0}(f)-T^{1}(\xi f)\right)=0
$$

by the condition (i) above. We write $C^{*}(T)$ for denoting the $C^{*}$-algebra generated by the images of the maps $T^{0}$ and $T^{1}$. Let $n$ be an integer greater than 1 , and $\xi_{1}, \ldots, \xi_{n}, \eta_{1}, \ldots, \eta_{n}$ be elements of $C_{d}\left(E^{1}\right)$. Set $\xi=\xi_{n} \otimes \cdots \otimes \xi_{1}, \eta=\eta_{n} \otimes \cdots \otimes \eta_{1} \in$ $C_{d}\left(E^{n}\right)$. By using conditions (i) and (ii) in Definition 2.2. we can prove

$$
\left(T^{1}\left(\xi_{n}\right) \cdots T^{1}\left(\xi_{1}\right)\right)^{*}\left(T^{1}\left(\eta_{n}\right) \cdots T^{1}\left(\eta_{1}\right)\right)=T^{0}(\langle\xi, \eta\rangle) .
$$

Therefore we can define a norm-decreasing linear map $T^{n}: C_{d}\left(E^{n}\right) \rightarrow C^{*}(T)$ by $T^{n}(\xi)=T^{1}\left(\xi_{n}\right) \cdots T^{1}\left(\xi_{1}\right)$ for $\xi=\xi_{n} \otimes \cdots \otimes \xi_{1} \in C_{d}\left(E^{n}\right)$. For $n \in \mathbb{N}$, we define a linear map $\Phi^{n}$ from $\operatorname{span}\left\{\theta_{\xi, \eta} \in \mathcal{K}\left(C_{d}\left(E^{n}\right)\right) \mid \xi, \eta \in C_{d}\left(E^{n}\right)\right\}$ to $C^{*}(T)$ by

$$
\Phi^{n}\left(\theta_{\xi, \eta}\right)=T^{n}(\xi) T^{n}(\eta)^{*} .
$$

One can check that this map is a well-defined norm-decreasing $*$-homomorphism (see [P Lemma 3.2] or [KPW Lemma 2.1]). Hence it uniquely extends to a *-homomorphism $\Phi^{n}: \mathcal{K}\left(C_{d}\left(E^{n}\right)\right) \rightarrow C^{*}(T)$. Note that $\Phi^{0}=T^{0}$ if we identify $\mathcal{K}\left(C_{d}\left(E^{0}\right)\right)$ with $C_{0}\left(E^{0}\right)$ in the natural way. We summarize properties of $T^{n}$ and $\Phi^{n}$ in the following lemma. The proof is left to the reader.

Lemma 2.3. Let $E=\left(E^{0}, E^{1}, d, r\right)$ be a topological graph and $T=\left(T^{0}, T^{1}\right)$ be a Toeplitz E-pair. Then the maps $T^{n}: C_{d}\left(E^{n}\right) \rightarrow C^{*}(T)$ and $\Phi^{n}: \mathcal{K}\left(C_{d}\left(E^{n}\right)\right) \rightarrow$ $C^{*}(T)$ defined above satisfy the following $\left(n, m \in \mathbb{N}, \xi, \zeta \in C_{d}\left(E^{n}\right), \eta \in C_{d}\left(E^{m}\right), f \in\right.$ $\left.C_{0}\left(E^{0}\right), x \in \mathcal{K}\left(C_{d}\left(E^{n}\right)\right)\right)$ :

(i) $T^{n}(\xi) T^{m}(\eta)=T^{n+m}(\xi \otimes \eta)$,

(ii) $T^{n}(\zeta)^{*} T^{n}(\xi)=T^{0}(\langle\zeta, \xi\rangle)$, 
(iii) $T^{0}(f) T^{n}(\xi)=T^{n}\left(\pi_{r^{n}}(f) \xi\right)$,

(iv) $T^{0}(f) \Phi^{n}(x)=\Phi^{n}\left(\pi_{r^{n}}(f) x\right)$,

(v) $\Phi^{n}(x) T^{n}(\xi)=T^{n}(x \xi)$.

We say that a Toeplitz $E$-pair $T=\left(T^{0}, T^{1}\right)$ is injective if $T^{0}$ is injective. It is easy to see that for an injective Toeplitz $E$-pair $T, T^{n}$ and $\Phi^{n}$ are isometric for all $n \in \mathbb{N}$.

Lemma 2.4. Let $n, m \in \mathbb{N}$ be integers with $n<m$, and $\xi \in C_{d}\left(E^{n}\right), \eta \in C_{d}\left(E^{m}\right)$. Then we have $T^{k}(\zeta)=T^{n}(\xi)^{*} T^{m}(\eta)$ where $k=m-n \in \mathbb{N}$ and $\zeta \in C_{d}\left(E^{k}\right)$ is defined by

$$
\zeta(e)=\sum_{\substack{e^{\prime} \in E^{n} \\ d\left(e^{\prime}\right)=r(e)}} \overline{\xi\left(e^{\prime}\right)} \eta\left(e^{\prime}, e\right) \quad\left(e \in E^{k}\right) .
$$

Proof. Take $\xi, \eta_{1} \in C_{d}\left(E^{n}\right)$ and $\eta_{2} \in C_{d}\left(E^{k}\right)$, and set $\eta=\eta_{1} \otimes \eta_{2} \in C_{d}\left(E^{m}\right)$. The element $\zeta \in C_{d}\left(E^{k}\right)$ defined by the above equation satisfies $\zeta=\pi_{r}\left(\left\langle\xi, \eta_{1}\right\rangle\right) \eta_{2}$ because we have

$$
\zeta(e)=\sum_{\substack{e^{\prime} \in E^{n} \\ d\left(e^{\prime}\right)=r(e)}} \overline{\xi\left(e^{\prime}\right)} \eta_{1}\left(e^{\prime}\right) \eta_{2}(e)=\left\langle\xi, \eta_{1}\right\rangle(r(e)) \eta_{2}(e)=\left(\pi_{r}\left(\left\langle\xi, \eta_{1}\right\rangle\right) \eta_{2}\right)(e),
$$

for $e \in E^{k}$. By Lemma 2.3 we get

$$
\begin{aligned}
T^{n}(\xi)^{*} T^{m}(\eta) & =T^{n}(\xi)^{*} T^{n}\left(\eta_{1}\right) T^{k}\left(\eta_{1}\right) \\
& =T^{0}\left(\left\langle\xi, \eta_{1}\right\rangle\right) T^{k}\left(\eta_{1}\right) \\
& =T^{k}\left(\pi_{r}\left(\left\langle\xi, \eta_{1}\right\rangle\right) \eta_{2}\right) \\
& =T^{k}(\zeta) .
\end{aligned}
$$

The set of linear combinations of elements of the form $\eta_{1} \otimes \eta_{2}\left(\eta_{1} \in C_{d}\left(E^{n}\right)\right.$, $\left.\eta_{2} \in C_{d}\left(E^{k}\right)\right)$ is dense in $C_{d}\left(E^{m}\right)$. Hence the equation holds for all $\xi \in C_{d}\left(E^{n}\right)$ and $\eta \in C_{d}\left(E^{m}\right)$.

By the above lemma, we have

$$
C^{*}(T)=\overline{\operatorname{span}}\left\{T^{n}(\xi) T^{m}(\eta)^{*} \mid \xi \in C_{d}\left(E^{n}\right), \eta \in C_{d}\left(E^{m}\right), n, m \in \mathbb{N}\right\} .
$$

Combining this fact with Lemma 1.20, we can easily show that the hereditary $C^{*}$ algebra generated by $T^{0}\left(C_{0}\left(E^{0}\right)\right) \subset C^{*}(T)$ is $C^{*}(T)$. From this fact, we get the following.

Proposition 2.5. A net $\left\{u_{i}\right\}$ in the multiplier algebra $\mathcal{M}\left(C^{*}(T)\right)$ of $C^{*}(T)$ converges in the strict topology if and only if $u_{i} T_{0}(f)$ and $T_{0}(f) u_{i}$ converge to elements in $C^{*}(T)$ in the norm topology for every $f \in C_{0}\left(E^{0}\right)$.

To introduce Cuntz-Krieger $E$-pairs of a graph $E$, we need the following notion.

Definition 2.6. Let $E=\left(E^{0}, E^{1}, d, r\right)$ be a topological graph. We define three open subsets $E_{\mathrm{sce}}^{0}, E_{\mathrm{fin}}^{0}$ and $E_{\mathrm{rg}}^{0}$ of $E^{0}$ by $E_{\mathrm{sce}}^{0}=E^{0} \backslash \overline{r\left(E^{1}\right)}$,

$$
E_{\text {fin }}^{0}=\left\{v \in E^{0} \mid \text { there exists a neighborhood } V \text { of } v\right.
$$
such that $r^{-1}(V) \subset E^{1}$ is compact $\}$,

and $E_{\mathrm{rg}}^{0}=E_{\mathrm{fin}}^{0} \backslash \overline{E_{\mathrm{sce}}^{0}}$. We define two closed subsets $E_{\mathrm{inf}}^{0}$ and $E_{\mathrm{sg}}^{0}$ of $E^{0}$ by $E_{\mathrm{inf}}^{0}=$ $E^{0} \backslash E_{\text {fin }}^{0}$ and $E_{\mathrm{sg}}^{0}=E^{0} \backslash E_{\mathrm{rg}}^{0}$. 
A vertex in $E_{\mathrm{sce}}^{0}$ is called a source. When $E$ is a discrete graph, $E_{\mathrm{fin}}^{0}$ is the set of vertices which receive finitely many edges, while $E_{\text {inf }}^{0}$ is the set of vertices which receive infinitely many edges. A vertex in $E_{\mathrm{rg}}^{0}$ is said to be regular, and a vertex in $E_{\mathrm{sg}}^{0}$ is said to be singular. We see that $E_{\mathrm{sg}}^{0}=\overline{E_{\mathrm{sce}}^{0}} \cup E_{\mathrm{inf}}^{0}$. Since $E_{\mathrm{sce}}^{0} \subset E_{\mathrm{fin}}^{0}$, we have $E_{\text {sce }}^{0} \cap E_{\text {inf }}^{0}=\emptyset$. However it may happen that $\overline{E_{\text {sce }}^{0}} \cap E_{\text {inf }}^{0} \neq \emptyset$, as the following example shows.

Example 2.7. Define a topological graph $E=\left(E^{0}, E^{1}, d, r\right)$ by $E^{0}=\mathbb{R}, E^{1}=$ $(0, \infty) \subset \mathbb{R}$ and $d, r$ are natural embeddings. Then we have $E_{\text {sce }}^{0}=(-\infty, 0), E_{\text {fin }}^{0}=$ $\mathbb{R} \backslash\{0\}$. Hence $E_{\text {inf }}^{0}=\{0\}$ and $\overline{E_{\text {sce }}^{0}}=(-\infty, 0]$ have a non-empty intersection. The set of regular vertices is $E_{\mathrm{rg}}^{0}=(0, \infty)$.

Proposition 2.8. For $v \in E^{0}$, we have $v \in E_{\mathrm{rg}}^{0}$ if and only if there exists a neighborhood $V$ of $v$ such that $r^{-1}(V)$ is compact and $r\left(r^{-1}(V)\right)=V$.

Proof. If there exists a neighborhood $V$ of $v$ such that $r^{-1}(V)$ is compact and $r\left(r^{-1}(V)\right)=V$, then $v \in E_{\text {fin }}^{0}$ and $V \cap E_{\mathrm{sce}}^{0}=\emptyset$. Hence we get $v \in E_{\mathrm{rg}}^{0}$. Conversely if $v \in E_{\mathrm{rg}}^{0}$, then a compact neighborhood $V$ of $v$ with $V \subset E_{\mathrm{rg}}^{0}$ has the property that $r^{-1}(V)$ is compact by Lemma 1.23 and that $r\left(r^{-1}(V)\right)=V$ by Lemma 1.22,

Proposition 2.8 means that the open set $E_{\mathrm{rg}}^{0}$ is the largest among the open subsets $U$ of $E^{0}$ such that the restriction of $r$ to $r^{-1}(U)$ is a proper surjection onto $U$. Note that for $v \in E_{\mathrm{rg}}^{0}, r^{-1}(v)$ is a non-empty compact set by Proposition 2.8

Definition 2.9. Let $E=\left(E^{0}, E^{1}, d, r\right)$ be a topological graph. A Toeplitz $E$-pair $T=\left(T^{0}, T^{1}\right)$ is called a Cuntz-Krieger E-pair if $T^{0}(f)=\Phi^{1}\left(\pi_{r}(f)\right)$ holds for all $f \in C_{0}\left(E_{\mathrm{rg}}^{0}\right)$.

Note that the restriction of $\pi_{r}$ to $C_{0}\left(E_{\mathrm{rg}}^{0}\right)$ is an injection into $\mathcal{K}\left(C_{d}\left(E^{1}\right)\right)$ by Proposition 1.24

Definition 2.10. We denote by $\mathcal{O}(E)$ the universal $C^{*}$-algebra generated by a Cuntz-Krieger $E$-pair $t=\left(t^{0}, t^{1}\right)$.

For $n \in \mathbb{N}$, we write $t^{n}: C_{d}\left(E^{n}\right) \rightarrow \mathcal{O}(E)$ and $\varphi^{n}: \mathcal{K}\left(C_{d}\left(E^{n}\right)\right) \rightarrow \mathcal{O}(E)$ for denoting the maps corresponding to $T^{n}$ and $\Phi^{n}$. For a Cuntz-Krieger $E$-pair $T=$ $\left(T^{0}, T^{1}\right)$, we denote by $\rho_{T}$ the unique surjection from $\mathcal{O}(E)$ to $C^{*}(T)$ satisfying $\rho_{T} \circ t^{i}=T^{i}$ for $i=0,1$. The map $\rho_{T}$ satisfies $\rho_{T} \circ t^{n}=T^{n}$ and $\rho_{T} \circ \varphi^{n}=\Phi^{n}$ for all $n \in \mathbb{N}$. At this point, we do not know whether there exists an injective Cuntz-Krieger $E$-pair, or even an injective Toeplitz $E$-pair. In the next section, we will construct one injective Cuntz-Krieger $E$-pair $\tau=\left(\tau^{0}, \tau^{1}\right)$. This implies that the universal Cuntz-Krieger $E$-pair $t=\left(t^{0}, t^{1}\right)$ is injective. The next lemma may help us to understand a role of $E_{\mathrm{rg}}^{0}$ and the definition of Cuntz-Krieger $E$-pairs.

Proposition 2.11. Let $T$ be an injective Toeplitz E-pair. If $f \in C_{0}\left(E^{0}\right)$ satisfies $T^{0}(f) \in \Phi^{1}\left(\mathcal{K}\left(C_{d}\left(E^{1}\right)\right)\right)$, then $f \in C_{0}\left(E_{\mathrm{rg}}^{0}\right)$ and $T^{0}(f)=\Phi^{1}\left(\pi_{r}(f)\right)$.

Proof. Let $f$ be an element of $C_{0}\left(E^{0}\right)$ satisfying $T^{0}(f) \in \Phi^{1}\left(\mathcal{K}\left(C_{d}\left(E^{1}\right)\right)\right)$. Take $x \in \mathcal{K}\left(C_{d}\left(E^{1}\right)\right)$ with $T^{0}(f)=\Phi^{1}(x)$. For $\xi \in C_{d}\left(E^{1}\right)$, we see that

$$
T^{1}\left(\pi_{r}(f) \xi\right)=T^{0}(f) T^{1}(\xi)=\Phi^{1}(x) T^{1}(\xi)=T^{1}(x \xi) .
$$

Since $T^{1}$ is injective, we get $\pi_{r}(f)=x$. Thus we have $T^{0}(f)=\Phi^{1}\left(\pi_{r}(f)\right)$. By Proposition 1.24, we have $f \in C_{0}\left(E_{\text {fin }}^{0}\right)$. We will show that $f \in C_{0}\left(E_{\mathrm{rg}}^{0}\right)$. To 
derive a contradiction, assume that there exists $v \notin E_{\mathrm{rg}}^{0}$ such that $f(v) \neq 0$. Since $f \in C_{0}\left(E_{\text {fin }}^{0}\right)$, we see that $v \in \overline{E_{\text {sce }}^{0}}$. Hence we can find $v^{\prime} \in E_{\text {sce }}^{0}$ such that $f\left(v^{\prime}\right) \neq 0$. The element $v^{\prime} \in E_{\text {sce }}^{0}$ has a neighborhood $V$ with $r^{-1}(V)=\emptyset$. Take $g \in C_{0}(V)$ with $g\left(v^{\prime}\right) \neq 0$. Then we have $f g \neq 0$ and

$$
T^{0}(f g)=T^{0}(f) T^{0}(g)=\Phi^{1}\left(\pi_{r}(f)\right) T^{0}(g)=\Phi^{1}\left(\pi_{r}(f) \pi_{r}(g)\right)=0,
$$

because $\pi_{r}(g)=0$. This contradicts the fact that $T^{0}$ is injective. Therefore $f \in$ $C_{0}\left(E_{\mathrm{rg}}^{0}\right)$.

Remark 2.12. In our sequel $\mathrm{Ka2}$, we will show that $\mathcal{O}(E)$ is the smallest $C^{*}$-algebra which is generated by an injective Toeplitz $E$-pair which admits a gauge action (which means that there exists an automorphism $\beta_{z}^{\prime}$ of $C^{*}(T)$ with $\beta_{z}^{\prime}\left(T^{0}(f)\right)=$ $T^{0}(f)$ and $\beta_{z}^{\prime}\left(T^{1}(\xi)\right)=z T^{1}(\xi)$ for every $\left.z \in \mathbb{T}\right)$.

We give two fundamental examples of topological graphs and $C^{*}$-algebras associated with them. More elaborated examples can be found in [Ka2].

Example 1 (graph algebras). When $E^{0}$ is discrete, $E^{1}$ is also discrete and $E=$ $\left(E^{0}, E^{1}, d, r\right)$ becomes an ordinary (directed) graph. We have

$$
\begin{aligned}
E_{\mathrm{fin}}^{0} & =\left\{v \in E^{0} \mid r^{-1}(v) \text { is a finite set }\right\}, \\
E_{\mathrm{sce}}^{0} & =\left\{v \in E^{0} \mid r^{-1}(v)=\emptyset\right\}, \\
E_{\mathrm{rg}}^{0} & =\left\{v \in E^{0} \mid r^{-1}(v) \text { is a non-empty finite set }\right\} .
\end{aligned}
$$

For a Toeplitz $E$-pair $\left(T^{0}, T^{1}\right)$, define $P_{v}=T^{0}\left(\delta_{v}\right)$ for $v \in E^{0}$ where $\delta_{v} \in C_{0}\left(E^{0}\right)$ is a characteristic function on $\{v\}$. Then $\left\{P_{v}\right\}_{v \in E^{0}}$ is a family of mutually orthogonal projections. Similarly set $S_{e}=T^{1}\left(\delta_{e}\right)$ for $e \in E^{1}$. Then the family $\left(\left\{P_{v}\right\}_{v \in E^{0}},\left\{S_{e}\right\}_{e \in E^{1}}\right)$ is a Toeplitz-Cuntz-Krieger $\bar{E}$-family in the sense of [FR] where $\bar{E}$ is the opposite graph of $E$, that is, the set of vertices and edges of $\bar{E}$ are the same as those of $E$, but the range map of $\bar{E}$ is $d$ and the source map of $\bar{E}$ is $r$. Conversely, from a Toeplitz-Cuntz-Krieger $\bar{E}$-family $\left(\left\{P_{v}\right\}_{v \in E^{0}},\left\{S_{e}\right\}_{e \in E^{1}}\right)$, we can define a Toeplitz $E$-pair $\left(T^{0}, T^{1}\right)$ by $T^{0}(f)=\sum_{v \in E^{0}} f(v) P_{v}$ and $T^{1}(\xi)=$ $\sum_{e \in E^{1}} \xi(e) S_{e}$. Thus there exists a one-to-one correspondence between the set of Toeplitz $E$-pairs and the set of Toeplitz-Cuntz-Krieger $\bar{E}$-families. Under this correspondence, Cuntz-Krieger $E$-pairs correspond exactly to Cuntz-Krieger $\bar{E}$-families in the sense of FLR. Thus $\mathcal{O}(E)$ is isomorphic to the graph algebra of the graph $\bar{E}$.

We can describe $\mathcal{K}\left(C_{d}\left(E^{1}\right)\right)$ explicitly in this case. For $e, e^{\prime} \in E^{1}$, we define $u_{e, e^{\prime}}=\theta_{\delta_{e}, \delta_{e^{\prime}}} \in \mathcal{K}\left(C_{d}\left(E^{1}\right)\right)$. Then we have

$$
\mathcal{K}\left(C_{d}\left(E^{1}\right)\right)=\overline{\operatorname{span}}\left\{u_{e, e^{\prime}} \mid e, e^{\prime} \in E^{1}\right\} .
$$

Lemma 2.13. We have $u_{e, e^{\prime}} \neq 0$ if and only if $d(e)=d\left(e^{\prime}\right)$.

Proof. When $e^{\prime \prime} \neq e^{\prime}$, we have $u_{e, e^{\prime}}\left(\delta_{e^{\prime \prime}}\right)=0$ because $\left\langle\delta_{e^{\prime}}, \delta_{e^{\prime \prime}}\right\rangle=0$. We have $u_{e, e^{\prime}}\left(\delta_{e^{\prime}}\right)=\delta_{e} \delta_{d\left(e^{\prime}\right)}$, and $\delta_{e} \delta_{d\left(e^{\prime}\right)}$ is non-zero if and only if $d(e)=d\left(e^{\prime}\right)$ (and in this case $\left.\delta_{e} \delta_{d\left(e^{\prime}\right)}=\delta_{e}\right)$. Hence $u_{e, e^{\prime}} \neq 0$ if and only if $d(e)=d\left(e^{\prime}\right)$.

Lemma 2.14. For $e_{1}, e_{1}^{\prime}, e_{2}, e_{2}^{\prime} \in E^{1}$ with $d\left(e_{1}\right)=d\left(e_{1}^{\prime}\right), d\left(e_{2}\right)=d\left(e_{2}^{\prime}\right)$, we have

$$
u_{e_{1}, e_{1}^{\prime}} u_{e_{2}, e_{2}^{\prime}}= \begin{cases}u_{e_{1}, e_{2}^{\prime}} & \text { if } e_{1}^{\prime}=e_{2}, \\ 0 & \text { if } e_{1}^{\prime} \neq e_{2} .\end{cases}
$$

Proof. Clear by the computation in the proof of Lemma 2.13. 
For $v \in E^{0}$, define $K_{v}=\overline{\operatorname{span}}\left\{u_{e, e^{\prime}} \mid e, e^{\prime} \in d^{-1}(v)\right\}$. By Lemma 2.13 and Lemma 2.14, we have the following.

Lemma 2.15. $\quad$ (i) If $d^{-1}(v)=\emptyset$, then $K_{v}=0$.

(ii) If $d^{-1}(v)$ is infinite, then $K_{v} \cong \mathbb{K}$.

(iii) If $d^{-1}(v)$ consists of $n$ edges, then $K_{v} \cong \mathbb{M}_{n}$.

(iv) For distinct $v, v^{\prime} \in E^{0}, K_{v}$ and $K_{v^{\prime}}$ are orthogonal to each other.

(v) $\mathcal{K}\left(C_{d}\left(E^{1}\right)\right)=\bigoplus_{v \in E^{0}} K_{v}$.

By Lemma2.15, it is easy to see that there exists a natural isomorphism between the $K$-groups of $\mathcal{K}\left(C_{d}\left(E^{1}\right)\right)$ and the ones of $C_{0}\left(d\left(E^{1}\right)\right)$. This is the case for general topological graphs $E$ because the Hilbert module $C_{d}\left(E^{1}\right)$ gives a strong Morita equivalence between $\mathcal{K}\left(C_{d}\left(E^{1}\right)\right)$ and $C_{0}\left(d\left(E^{1}\right)\right) \subset C_{0}\left(E^{0}\right)$ (see [E]). The map $\pi_{r}: C_{0}\left(E_{\text {fin }}^{0}\right) \rightarrow \mathcal{K}\left(C_{d}\left(E^{1}\right)\right)$ can be described as

$$
\pi_{r}(f)=\sum_{e \in E^{1}} f(r(e)) u_{e, e}=\bigoplus_{v \in E^{0}} \hat{r}(f)(v),
$$

where $\hat{r}(f)(v)=\sum_{e \in d^{-1}(v)} f(r(e)) u_{e, e}$ is a diagonal operator of $K_{v}$ for $v \in E^{0}$. This formula makes sense for the map $\pi_{r}: C_{0}\left(E^{0}\right) \rightarrow \mathcal{L}\left(C_{d}\left(E^{1}\right)\right)$, where infinite sums converge in strict topology.

Example 2 (homeomorphism $C^{*}$-algebras). Take a topological dynamical system $\Sigma=(X, \sigma)$ where $X$ is a compact space and $\sigma: X \rightarrow X$ is a homeomorphism. We can define an automorphism $\alpha$ of $C(X)$ by $\alpha(f)(x)=f\left(\sigma^{-1} x\right)$. The crossed product $C(X) \rtimes_{\alpha} \mathbb{Z}$ is called a homeomorphism $C^{*}$-algebra and denoted by $A(\Sigma)$ in T3. T4. A representation of $A(\Sigma)$ corresponds bijectively to a covariant representation $\{\pi, u\}$ of the topological dynamical system $\Sigma$ where $\pi$ is a representation of $C(X)$ and $u$ is a unitary satisfying $\pi(\alpha(f))=u \pi(f) u^{*}$. A homeomorphism $\sigma$ defines a topological correspondence on $X$, hence we get a topological graph $E_{\Sigma}$ from $\Sigma$. We will see that the homeomorphism $C^{*}$-algebra $A(\Sigma)$ is isomorphic to $\mathcal{O}\left(E_{\Sigma}\right)$.

We treat a more general setting, namely when $X$ is a locally compact space, and $\sigma$ is a proper continuous map from $X$ to $X$. Define a topological graph $E_{\Sigma}=$ $\left(E_{\Sigma}^{0}, E_{\Sigma}^{1}, d, r\right)$ by $E_{\Sigma}^{0}=E_{\Sigma}^{1}=X, d=\operatorname{id}_{X}$ and $r=\sigma$. For a natural number $n \geq 2$,

$$
E_{\Sigma}^{n}=\left\{\left(x_{n}, \ldots, x_{1}\right) \in X \times \cdots \times X \mid x_{k}=\sigma\left(x_{k-1}\right) \text { for } k=2, \ldots, n\right\}
$$

is homeomorphic to $X$ by $E_{\Sigma}^{n} \ni\left(x_{n}, \ldots, x_{1}\right) \mapsto x_{1} \in X$. We will identify $E_{\Sigma}^{n}$ with $X$ by this map. Under this identification, we see that $d^{n}=\operatorname{id}_{X}$ and $r^{n}=\sigma^{n}$. In other words, $\left(X, \operatorname{id}_{X}, \sigma^{n}\right)$ is the $n$-times composition of the topological correspondence $\left(X, \operatorname{id}_{X}, \sigma\right)$. We identify $C_{d^{n}}\left(E_{\Sigma}^{n}\right)$ with $C_{0}(X)$ for every $n \in \mathbb{N}$ as (right) Hilbert $C_{0}(X)$-modules. We define an endomorphism $\hat{\sigma}: C_{0}(X) \rightarrow C_{0}(X)$ by $\hat{\sigma}(f)=f \circ \sigma$. If we identify $\mathcal{K}\left(C_{d}\left(E_{\Sigma}^{1}\right)\right)$ with $C_{0}(X)$, then the map $\hat{\sigma}$ coincides with the left action $\pi_{r}: C_{0}(X) \rightarrow \mathcal{K}\left(C_{d}\left(E_{\Sigma}^{1}\right)\right) \subset \mathcal{L}\left(C_{d}\left(E_{\Sigma}^{1}\right)\right)$ defined by $r(=\sigma)$. Let us take a Toeplitz $E_{\Sigma}$-pair $T=\left(T^{0}, T^{1}\right)$.

Lemma 2.16. For $\xi, \eta \in C_{0}(X)$ and $n, m \in \mathbb{N}$, we have the following.

(i) $T^{n}(\xi)^{*} T^{n}(\eta)=T^{0}(\bar{\xi} \eta)$.

(ii) $T^{n}(\xi) T^{m}(\eta)=T^{n+m}\left(\hat{\sigma}^{m}(\xi) \eta\right)$.

(iii) $T^{n}(\xi)^{*} T^{m}(\eta)=T^{m-n}\left(\hat{\sigma}^{m-n}(\bar{\xi}) \eta\right)$ when $n \leq m$.

Proof. (i) Clear by $d^{n}=\operatorname{id}_{X}$. 
(ii) We have $T^{n}(\xi) T^{m}(\eta)=T^{n+m}(\xi \otimes \eta)$ and

$$
\xi \otimes \eta(x)=\xi \otimes \eta\left(\sigma^{n+m-1}(x), \ldots, \sigma(x), x\right)=\xi\left(\sigma^{m}(x)\right) \eta(x)=\left(\hat{\sigma}^{m}(\xi) \eta\right)(x) .
$$

Hence we have $T^{n}(\xi) T^{m}(\eta)=T^{n+m}\left(\hat{\sigma}^{m}(\xi) \eta\right)$.

(iii) Since the set $\left\{\hat{\sigma}^{m-n}\left(\eta_{1}\right) \eta_{2} \mid \eta_{1}, \eta_{2} \in C_{0}(X)\right\}$ is dense in $C_{0}(X)$, it suffices to show the equation for $\eta=\hat{\sigma}^{m-n}\left(\eta_{1}\right) \eta_{2}$ where $\eta_{1}, \eta_{2} \in C_{0}(X)$. We have $T^{m}(\eta)=$ $T^{n}\left(\eta_{1}\right) T^{m-n}\left(\eta_{2}\right)$ by (ii). Hence

$$
\begin{aligned}
T^{n}(\xi)^{*} T^{m}(\eta) & =T^{n}(\xi)^{*} T^{n}\left(\eta_{1}\right) T^{m-n}\left(\eta_{2}\right)=T^{0}\left(\bar{\xi} \eta_{1}\right) T^{m-n}\left(\eta_{2}\right) \\
& =T^{m-n}\left(\hat{\sigma}^{m-n}\left(\bar{\xi} \eta_{1}\right) \eta_{2}\right)=T^{m-n}\left(\hat{\sigma}^{m-n}(\bar{\xi}) \eta\right) .
\end{aligned}
$$

Proposition 2.17. Take an approximate unit $\left\{u_{i}\right\}$ of $C_{0}(X)$. Then the net $\left\{T^{1}\left(u_{i}\right)\right\}$ in $C^{*}(T)$ converges strictly to an element $U_{T} \in \mathcal{M}\left(C^{*}(T)\right)$ satisfying $U_{T} T^{0}(f)=$ $T^{1}(f)$ and $T^{0}(f) U_{T}=T^{1}(\hat{\sigma}(f))$ for $f \in C_{0}(X)$.

Proof. For $f \in C_{0}(X)$, we have $T^{1}\left(u_{i}\right) T^{0}(f)=T^{1}\left(u_{i} f\right)$ which converges to $T^{1}(f)$ in the norm topology. We also have that $T^{0}(f) T^{1}\left(u_{i}\right)=T^{1}\left(\hat{\sigma}(f) u_{i}\right)$ converges to $T^{1}(\hat{\sigma}(f))$ in the norm topology. Now the assertion follows from Proposition 2.5.

Proposition 2.18. The element $U_{T} \in \mathcal{M}\left(C^{*}(T)\right)$ defined in Proposition 2.17 satisfies the following.

(i) $U_{T}^{*} U_{T}=1$.

(ii) $T^{0}(f) U_{T}=U_{T} T^{0}(\hat{\sigma}(f))$ for $f \in C_{0}(X)$.

(iii) $T^{n}(f)=U_{T}^{n} T^{0}(f)$ for $f \in C_{0}(X)$.

Proof. (i) For an approximate unit $u_{i}$ of $C_{0}(X),\left|u_{i}\right|^{2}$ is also an approximate unit. Hence $T^{1}\left(u_{i}\right)^{*} T^{1}\left(u_{i}\right)=T^{0}\left(\left|u_{i}\right|^{2}\right)$ converges strictly to $1 \in \mathcal{M}\left(C^{*}(T)\right)$. Thus we have $U_{T}^{*} U_{T}=1$.

(ii) By Proposition 2.17, $T^{0}(f) U_{T}=T^{1}(\hat{\sigma}(f))=U_{T} T^{0}(\hat{\sigma}(f))$.

(iii) Similarly to the proof of Proposition [2.17, we have $U_{T} T^{n-1}(f)=T^{n}(f)$. Hence we get $T^{n}(f)=U_{T}^{n} T^{0}(f)$.

Thus from a Toeplitz $E_{\Sigma}$-pair $T$, we get an isometry $U_{T} \in \mathcal{M}\left(C^{*}(T)\right)$ satisfying $T^{0}(f) U_{T}=U_{T} T^{0}(\hat{\sigma}(f))$. Conversely, we have the following.

Proposition 2.19. Let $A$ be a $C^{*}$-algebra. Suppose that a $*$-homomorphism $\pi$ : $C_{0}(X) \rightarrow A$ and an isometry $u \in \mathcal{M}(A)$ satisfy $\pi(f) u=u \pi(\hat{\sigma}(f))$ for all $f \in$ $C_{0}(X)$. Define $T^{0}, T^{1}: C_{0}(X) \rightarrow A$ by $T^{0}=\pi$ and $T^{1}(f)=u \pi(f)$. Then $T=$ $\left(T^{0}, T^{1}\right)$ is a Toeplitz $E_{\Sigma}$-pair.

Proof. Since $u$ is an isometry, $T$ satisfies the condition (i) in Definition 2.2. The condition (ii) is easily checked from the relation $\pi(f) u=u \pi(\hat{\sigma}(f))$. Thus $T=$ $\left(T^{0}, T^{1}\right)$ is a Toeplitz $E_{\Sigma}$-pair.

A pair $\{\pi, u\}$ that appeared in Proposition [2.19] can be considered as a kind of covariant representations of $(X, \sigma)$. These representations correspond to Toeplitz $E_{\Sigma}$-pairs. We study which representation $\{\pi, u\}$ corresponds to a Cuntz-Krieger $E_{\Sigma}$-pair. Since $\sigma$ is proper, we have $\left(E_{\Sigma}^{0}\right)_{\text {fin }}=X$ and $\left(E_{\Sigma}^{0}\right)_{\text {sce }}=X \backslash \sigma(X)$. Hence $\left(E_{\Sigma}^{0}\right)_{\mathrm{rg}}=X \backslash \overline{X \backslash \sigma(X)}$ which is the interior of the image $\sigma(X)$ of $\sigma$. It is not difficult to see that the map $\Phi^{1}: \mathcal{K}\left(C_{d}\left(E_{\Sigma}^{1}\right)\right) \rightarrow C^{*}(T)$ can be expressed as $\Phi^{1}(f)=$ $U_{T} T^{0}(f) U_{T}^{*}$ for $f \in C_{0}(X)$ by identifying $\mathcal{K}\left(C_{d}\left(E_{\Sigma}^{1}\right)\right)$ with $C_{0}(X)$. Hence we have 
$\Phi^{1}\left(\pi_{r}(f)\right)=U_{T} T^{0}(\hat{\sigma}(f)) U_{T}^{*}$ for $f \in C_{0}(X)$. We write $P_{T}=1-U_{T} U_{T}^{*} \in \mathcal{M}\left(C^{*}(T)\right)$ which is a projection. For $f \in C_{0}(X)$, we have

$$
T^{0}(f) P_{T}=T^{0}(f)-U_{T} T^{0}(\hat{\sigma}(f)) U_{T}^{*}=P_{T} T^{0}(f)
$$

by Proposition 2.18 (ii). By the above argument, we have the following.

Proposition 2.20. For a Toeplitz $E_{\Sigma}$-pair $T$, the following are equivalent.

(i) $T$ is a Cuntz-Krieger $E_{\Sigma}$-pair.

(ii) $T^{0}(f)=U_{T} T^{0}(\hat{\sigma}(f)) U_{T}^{*}$ for all $f \in C_{0}(X)$ with $f(x)=0$ for $x \notin \sigma(X)$.

(iii) $T^{0}(f) P_{T}=0$ for all $f \in C_{0}(X)$ with $f(x)=0$ for $x \notin \sigma(X)$.

Proposition 2.21. The $C^{*}$-algebra $\mathcal{O}\left(E_{\Sigma}\right)$ is the universal $C^{*}$-algebra generated by products of a copy of $C_{0}(X)$ and an isometry $u$ satisfying

(i) $f u=u \hat{\sigma}(f)$ for $f \in C_{0}(X)$,

(ii) $f=u \hat{\sigma}(f) u^{*}$ for $f \in C_{0}(X)$ with $f(x)=0$ for $x \notin \sigma(X)$.

When $\sigma$ is surjective, for a Cuntz-Krieger $E_{\Sigma}$-pair $T$ we have $P_{T}=0$, that is, $U_{T}$ is a unitary. Hence, we have the following.

Corollary 2.22. When $\sigma$ is surjective, the $C^{*}$-algebra $\mathcal{O}\left(E_{\Sigma}\right)$ is the universal $C^{*}$-algebra generated by products of a copy of $C_{0}(X)$ and a unitary $u$ satisfying $\hat{\sigma}(f)=u^{*}$ fu for $f \in C_{0}(X)$.

Corollary 2.23. When $\sigma$ is a homeomorphism, Cuntz-Krieger $E_{\Sigma}$-pairs correspond to covariant representations of the dynamical system $\Sigma$, and we have a natural isomorphism between $\mathcal{O}\left(E_{\Sigma}\right)$ and the homeomorphism $C^{*}$-algebra $A(\Sigma)$.

It is complicated to describe Toeplitz $E_{\Sigma}$-pairs or Cuntz-Krieger $E_{\Sigma}$-pairs when $\sigma$ is not proper, or when $\sigma$ is defined just on some open subset of $X$.

\section{Fock Representations}

The purpose of this section is a construction of an injective Cuntz-Krieger $E$ pair $\tau=\left(\tau^{0}, \tau^{1}\right)$ of a graph $E$ by using the so-called Fock space $C_{d}\left(E^{*}\right)$. The map $\rho_{\tau}: \mathcal{O}(E) \rightarrow C^{*}(\tau)$ is called the Fock representation. In the next section, it will turn out that the Fock representation is faithful. Hence the construction done in this section gives us a concrete description of $\mathcal{O}(E)$. The results here will be used in Section 6 to compute $K$-groups.

Definition 3.1. For a topological graph $E=\left(E^{0}, E^{1}, d, r\right)$, we denote by $E^{*}$ the disjoint union of $E^{0}, E^{1}, \ldots, E^{n}, \ldots$.

The set $E^{*}$ is called a finite path space of a topological graph $E$. We can define $d, r: E^{*} \rightarrow E^{0}$ by using $d^{n}, r^{n}$. The $C^{*}$-correspondence $C_{d}\left(E^{*}\right)$ over $C_{0}\left(E^{0}\right)$, which is called a Fock space, is isomorphic to $\bigoplus_{n=0}^{\infty} C_{d^{n}}\left(E^{n}\right)$. We denote the left action of $C_{0}\left(E^{0}\right)$ on $C_{d}\left(E^{*}\right)$ by $\sigma^{0}: C_{0}\left(E^{0}\right) \rightarrow \mathcal{L}\left(C_{d}\left(E^{*}\right)\right)$. Explicitly, for $f \in C_{0}\left(E^{0}\right)$ we see that

$$
\begin{cases}\sigma^{0}(f) \xi=f \xi, & \text { for } \xi \in C_{d^{0}}\left(E^{0}\right) \subset C_{d}\left(E^{*}\right), \\ \sigma^{0}(f)(\xi \otimes \eta)=\left(\pi_{r}(f) \xi\right) \otimes \eta, & \text { for } \xi \in C_{d^{1}}\left(E^{1}\right), \eta \in C_{d^{n}}\left(E^{n}\right)(n \in \mathbb{N}),\end{cases}
$$

where $\pi_{r}$ is the left action of $C_{0}\left(E^{0}\right)$ on $C_{d}\left(E^{1}\right)$ defined in Section 2. We define a linear map $\sigma^{1}: C_{d}\left(E^{1}\right) \ni \xi \mapsto \sigma^{1}(\xi) \in \mathcal{L}\left(C_{d}\left(E^{*}\right)\right)$ by $\sigma^{1}(\xi) \eta=\xi \otimes \eta \in C_{d^{n+1}}\left(E^{n+1}\right)$ 
for $\eta \in C_{d^{n}}\left(E^{n}\right) \subset C_{d}\left(E^{*}\right)$. The routine computation shows the following formulae of the adjoint $\sigma^{1}\left(\xi_{0}\right)^{*}$ of $\sigma^{1}\left(\xi_{0}\right)$ for $\xi_{0} \in C_{d}\left(E^{1}\right)$ :

$$
\begin{cases}\sigma^{1}\left(\xi_{0}\right)^{*} \xi=0, & \text { for } \xi \in C_{d^{0}}\left(E^{0}\right), \\ \sigma^{1}\left(\xi_{0}\right)^{*}(\xi \otimes \eta)=\sigma^{0}\left(\left\langle\xi_{0}, \xi\right\rangle\right) \eta, & \text { for } \xi \in C_{d^{1}}\left(E^{1}\right), \eta \in C_{d^{n}}\left(E^{n}\right)(n \in \mathbb{N}) .\end{cases}
$$

Now, it is easy to see the following.

Proposition 3.2 (cf. [P, Proposition 1.3]). The pair $\sigma=\left(\sigma^{0}, \sigma^{1}\right)$ is a Toeplitz E-pair.

Recall that the map $\Phi^{1}: \mathcal{K}\left(C_{d}\left(E^{1}\right)\right) \rightarrow C^{*}(\sigma) \subset \mathcal{L}\left(C_{d}\left(E^{*}\right)\right)$ is defined by $\Phi^{1}\left(\theta_{\xi, \eta}\right)$ $=\sigma^{1}(\xi) \sigma^{1}(\eta)^{*}$. For $x \in \mathcal{K}\left(C_{d}\left(E^{1}\right)\right)$, we see that

$$
\begin{cases}\Phi^{1}(x) \xi=0, & \text { for } \xi \in C_{d^{0}}\left(E^{0}\right), \\ \Phi^{1}(x)(\xi \otimes \eta)=(x \xi) \otimes \eta, & \text { for } \xi \in C_{d^{1}}\left(E^{1}\right), \eta \in C_{d^{n}}\left(E^{n}\right)(n \in \mathbb{N}) .\end{cases}
$$

From this computation, we get the following lemma, which measures how far the Toeplitz $E$-pair $\sigma=\left(\sigma^{0}, \sigma^{1}\right)$ is from being a Cuntz-Krieger $E$-pair.

Lemma 3.3. For $f \in C_{0}\left(E_{\mathrm{rg}}^{0}\right)$, take $\xi_{0}, \eta_{0} \in C_{d^{0}}\left(E^{0}\right) \subset C_{d}\left(E^{*}\right)$ with $\xi_{0} \overline{\eta_{0}}=f$. Then we have $\theta_{\xi_{0}, \eta_{0}}=\sigma^{0}(f)-\Phi^{1}\left(\pi_{r}(f)\right)$.

Proof. By the computation above, it suffices to show that $\theta_{\xi_{0}, \eta_{0}}(\xi)=f \xi$ if $\xi \in$ $C_{d^{0}}\left(E^{0}\right) \subset C_{d}\left(E^{*}\right)$, and $\theta_{\xi_{0}, \eta_{0}}(\xi)=0$ if $\xi \in C_{d^{n}}\left(E^{n}\right) \subset C_{d}\left(E^{*}\right)$ for $n \geq 1$. The former is verified by

$$
\theta_{\xi_{0}, \eta_{0}}(\xi)=\xi_{0} \overline{\eta_{0}} \xi=f \xi
$$

and the latter is obvious. The proof is completed.

For each $n \in \mathbb{N}$, we define an open subset $E_{\mathrm{rg}}^{n}$ of $E^{n}$ by $E_{\mathrm{rg}}^{n}=\left(d^{n}\right)^{-1}\left(E_{\mathrm{rg}}^{0}\right)$ and an open subset $E_{\mathrm{rg}}^{*}$ of $E^{*}$ by $E_{\mathrm{rg}}^{*}=d^{-1}\left(E_{\mathrm{rg}}^{0}\right)$. Note that $C_{d}\left(E_{\mathrm{rg}}^{*}\right)=\bigoplus_{n=0}^{\infty} C_{d^{n}}\left(E_{\mathrm{rg}}^{n}\right) \subset$ $C_{d}\left(E^{*}\right)$ and that $\mathcal{K}\left(C_{d}\left(E_{\mathrm{rg}}^{*}\right)\right)$ is an ideal of $\mathcal{L}\left(C_{d}\left(E^{*}\right)\right)$.

Proposition 3.4. We have $\mathcal{K}\left(C_{d}\left(E_{\mathrm{rg}}^{*}\right)\right) \subset C^{*}(\sigma)$.

Proof. It suffices to show that $\theta_{\xi, \eta} \in C^{*}(\sigma)$ for $\xi \in C_{d^{n}}\left(E_{\mathrm{rg}}^{n}\right) \subset C_{d}\left(E_{\mathrm{rg}}^{*}\right)$ and $\eta \in C_{d^{m}}\left(E_{\mathrm{rg}}^{m}\right) \subset C_{d}\left(E_{\mathrm{rg}}^{*}\right)$ for $n, m \in \mathbb{N}$. By Lemma 1.12, we can find $\xi^{\prime} \in C_{d^{n}}\left(E^{n}\right)$, $\eta^{\prime} \in C_{d^{m}}\left(E^{m}\right)$ and $f, g \in C_{0}\left(E_{\mathrm{rg}}^{0}\right)$ with $\xi=\xi^{\prime} f$ and $\eta=\eta^{\prime} g$. Once we consider $f, g$ as elements of $C_{d^{0}}\left(E^{0}\right) \subset C_{d}\left(E^{*}\right)$, we have $\xi=\sigma^{n}\left(\xi^{\prime}\right) f$ and $\eta=\sigma^{m}\left(\eta^{\prime}\right) g$. By Lemma 3.3, we have that

$$
\theta_{f, g}=\sigma^{0}(h)-\Phi^{1}\left(\pi_{r}(h)\right) \in C^{*}(\sigma),
$$

where $h=f \bar{g} \in C_{0}\left(E_{\mathrm{rg}}^{0}\right)$. Hence we get

$$
\theta_{\xi, \eta}=\sigma^{n}\left(\xi^{\prime}\right) \theta_{f, g} \sigma^{m}\left(\eta^{\prime}\right)^{*} \in C^{*}(\sigma) .
$$

The proof is completed.

Let

and

$$
\tau^{0}: C_{0}\left(E^{0}\right) \rightarrow \mathcal{L}\left(C_{d}\left(E^{*}\right)\right) / \mathcal{K}\left(C_{d}\left(E_{\mathrm{rg}}^{*}\right)\right)
$$

$$
\tau^{1}: C_{d}\left(E^{1}\right) \rightarrow \mathcal{L}\left(C_{d}\left(E^{*}\right)\right) / \mathcal{K}\left(C_{d}\left(E_{\text {rg }}^{*}\right)\right)
$$

be the compositions of the natural surjection $\mathcal{L}\left(C_{d}\left(E^{*}\right)\right) \rightarrow \mathcal{L}\left(C_{d}\left(E^{*}\right)\right) / \mathcal{K}\left(C_{d}\left(E_{\text {rg }}^{*}\right)\right)$ with $\sigma^{0}$ and $\sigma^{1}$ respectively. By Lemma 3.3, the pair $\tau=\left(\tau^{0}, \tau^{1}\right)$ is a Cuntz-Krieger $E$-pair. We will show that this pair is injective. 
Lemma 3.5. Let $n$ be a natural number, and $f$ be an element of $C_{0}\left(E^{0}\right)$ with $\pi_{r^{n}}(f) \in \mathcal{K}\left(C_{d^{n}}\left(E_{\mathrm{rg}}^{n}\right)\right)$. If $e \in E^{n}$ satisfies $f\left(r^{n}(e)\right) \neq 0$, then $e \in E_{\mathrm{rg}}^{n}$.

Proof. To the contrary, assume that $e \in E^{n}$ satisfies $f\left(r^{n}(e)\right) \neq 0$ and $e \notin E_{\mathrm{rg}}^{n}$. Take $\xi_{1}, \ldots, \xi_{m}, \eta_{1}, \ldots, \eta_{m}$ in $C_{c}\left(E_{\mathrm{rg}}^{n}\right)$ arbitrarily, and we will show that

$$
\left\|\pi_{r}(f)-\sum_{k=1}^{m} \theta_{\xi_{k}, \eta_{k}}\right\| \geq\left|f\left(r^{n}(e)\right)\right|
$$

which contradicts the fact that $\pi_{r^{n}}(f) \in \mathcal{K}\left(C_{d^{n}}\left(E_{\mathrm{rg}}^{n}\right)\right)$. Set $X=\bigcup_{k=1}^{m} \operatorname{supp}\left(\eta_{k}\right)$ which is a compact set with $X \subset E_{\mathrm{rg}}^{n}$. We can find a neighborhood $U$ of $e$ such that $X \cap U=\emptyset$ and that the restriction of $d^{n}$ to $U$ is injective. Take $\zeta \in C_{c}(U) \subset$ $C_{d^{n}}\left(E^{n}\right)$ with $0 \leq \zeta \leq 1$ and $\zeta(e)=1$. Similarly to the proof of Proposition 1.17 we have that $\|\zeta\|=1$ and

$$
\left\|\left(\pi_{r^{n}}(f)-\sum_{k=1}^{m} \theta_{\xi_{k}, \eta_{k}}\right) \zeta\right\|=\left\|\pi_{r^{n}}(f) \zeta\right\| \geq\left|f\left(r^{n}(e)\right)\right| .
$$

We are done.

Proposition 3.6. The Cuntz-Krieger E-pair $\tau=\left(\tau^{0}, \tau^{1}\right)$ is injective.

Proof. To the contrary, assume that there exists $f \in C_{0}\left(E^{0}\right)$ with $f \neq 0$ and $\sigma^{0}(f) \in$ $\mathcal{K}\left(C_{d}\left(E_{\mathrm{rg}}^{*}\right)\right)$. The fact $\sigma^{0}(f) \in \mathcal{K}\left(C_{d}\left(E_{\mathrm{rg}}^{*}\right)\right)$ implies that $\pi_{r^{n}}(f) \in \mathcal{K}\left(C_{d^{n}}\left(E_{\mathrm{rg}}^{n}\right)\right)$ for every $n \in \mathbb{N}$. We can find $\varepsilon>0$ and a non-empty open subset $V$ of $E^{0}$ such that $|f(v)| \geq \varepsilon$ for any $v \in V$. We will show that $\left(r^{n}\right)^{-1}(V)$ is a non-empty subset of $E_{\mathrm{rg}}^{n}$ for every $n \in \mathbb{N}$ by induction. For $n=0$, we have $V \subset E_{\mathrm{rg}}^{0}$ because $\pi_{r^{0}}(f) \in \mathcal{K}\left(C_{d^{0}}\left(E_{\mathrm{rg}}^{0}\right)\right) \cong C_{0}\left(E_{\mathrm{rg}}^{0}\right)$. Assume that $\left(r^{n}\right)^{-1}(V)$ is a non-empty subset of $E_{\mathrm{rg}}^{n}$. Then we have $d^{n}\left(\left(r^{n}\right)^{-1}(V)\right) \subset E_{\mathrm{rg}}^{0} \subset \overline{r\left(E^{1}\right)}$. Since $d^{n}\left(\left(r^{n}\right)^{-1}(V)\right)$ is non-empty and open, there exists $e \in\left(r^{n}\right)^{-1}(V)$ such that $d^{n}(e) \in r\left(E^{1}\right)$. Hence $\left(r^{n+1}\right)^{-1}(V)$ is non-empty. Since $\left|f\left(r^{n+1}(e)\right)\right| \geq \varepsilon$ for $e \in\left(r^{n+1}\right)^{-1}(V)$, we have $\left(r^{n+1}\right)^{-1}(V) \subset E_{\mathrm{rg}}^{n+1}$ by Lemma 3.5. Thus we have shown that $\left(r^{n}\right)^{-1}(V)$ is a nonempty subset of $E_{\mathrm{rg}}^{n}$ for every $n \in \mathbb{N}$. We will show that $\left\|\sigma^{0}(f)-\sum_{k=1}^{m} \theta_{\xi_{k}, \eta_{k}}\right\| \geq \varepsilon$ for any $\xi_{k}, \eta_{k} \in C_{d}\left(E_{\mathrm{rg}}^{*}\right)$, which contradicts the fact that $\sigma^{0}(f) \in \mathcal{K}\left(C_{d}\left(E_{\mathrm{rg}}^{*}\right)\right)$. To this end, it suffices to find $\zeta_{n} \in C_{d^{n}}\left(E^{n}\right)$ with $\left\|\zeta_{n}\right\|=1,\left\|\sigma^{0}(f) \zeta_{n}\right\| \geq \varepsilon$ for each $n \in \mathbb{N}$. Since $\left(r^{n}\right)^{-1}(V)$ is not empty, we can find $\zeta_{n} \in C_{c}\left(\left(r^{n}\right)^{-1}(V)\right) \subset C_{d^{n}}\left(E^{n}\right)$ with $\left\|\zeta_{n}\right\|=1$. Since

$$
\left|\left(\sigma^{0}(f) \zeta_{n}\right)(e)\right|=\left|f\left(r^{n}(e)\right) \zeta_{n}(e)\right| \geq \varepsilon\left|\zeta_{n}(e)\right|
$$

for $e \in\left(r^{n}\right)^{-1}(V)$, we have $\left\|\sigma^{0}(f) \zeta_{n}\right\| \geq \varepsilon\left\|\zeta_{n}\right\|=\varepsilon$. We are done.

As claimed in the previous section, Proposition 3.6]implies the following.

Proposition 3.7. The universal Cuntz-Krieger E-pair $t=\left(t^{0}, t^{1}\right)$ is injective.

The map $\rho_{\tau}: \mathcal{O}(E) \rightarrow C^{*}(\tau)$ is called the Fock representation. In the next section, we will show that the Fock representation gives us an isomorphism $C^{*}(\tau) \cong$ $\mathcal{O}(E)$ (Corollary 4.7).

We finish this section by stating a relation between our $C^{*}$-algebras $\mathcal{T}(E), \mathcal{O}(E)$ and ones defined in $\left[\mathrm{P}\right.$. The $C^{*}$-algebra $C^{*}(\sigma) \subset \mathcal{L}\left(C_{d}\left(E^{*}\right)\right)$ is exactly the same as the augmented Toeplitz algebra $\widetilde{\mathcal{T}}_{C_{d}\left(E^{1}\right)}$ of the $C^{*}$-correspondence $C_{d}\left(E^{1}\right)$ over $C_{0}\left(E^{0}\right)$ defined in $[\mathrm{P}$, Remark $1.2(3)]$. Hence Theorem 3.4 of $[\mathrm{P}$ gives the following because the conditions there are the same as the ones of Toeplitz $E$-pairs. 
Proposition 3.8. The pair $\sigma=\left(\sigma^{0}, \sigma^{1}\right)$ is the universal Toeplitz E-pair. Hence $C^{*}(\sigma)$ is isomorphic to $\mathcal{T}(E)$.

Therefore we see that $\mathcal{T}(E)$ is isomorphic to the augmented Toeplitz algebra $\widetilde{\mathcal{T}}_{C_{d}\left(E^{1}\right)}$. Of course one can show Proposition 3.8 by using a similar argument in Section 4 (which is actually the same as the proof in $[\mathrm{P}]$ ). One can also deduce Proposition 3.8 from Theorem 4.5, by proving that Toeplitz pairs correspond bijectively to Cuntz-Krieger pairs of a certain topological graph (see [Ka2]).

The augmented Cuntz-Pimsner algebra $\widetilde{\mathcal{O}}_{C_{d}\left(E^{1}\right)}$ is isomorphic to the universal $C^{*}$-algebra generated by a Toeplitz $E$-pair $T=\left(T^{0}, T^{1}\right)$ satisfying $T^{0}(f)=$ $\Phi^{1}\left(\pi_{r}(f)\right)$ for every $f \in C_{0}\left(E_{\text {fin }}^{0}\right)$, not only $f \in C_{0}\left(E_{\mathrm{rg}}^{0}\right)$ (see $[\mathrm{P}$, Theorem 3.12]). Hence there exists a surjection $\mathcal{O}(E) \rightarrow \widetilde{\mathcal{O}}_{C_{d}\left(E^{1}\right)}$. In this sense, $\widetilde{\mathcal{O}}_{C_{d}\left(E^{1}\right)}$ is "smaller" than $\mathcal{O}(E)$. Sometimes $\widetilde{\mathcal{O}}_{C_{d}\left(E^{1}\right)}$ is too small and can be 0 . If there exists a source, then $t^{0}: C_{0}\left(E^{0}\right) \rightarrow \widetilde{\mathcal{O}}_{C_{d}\left(E^{1}\right)}$ never becomes injective because $\pi_{r}(f)=0$ for $f \in C_{0}\left(E_{\mathrm{sce}}^{0}\right)$ by Proposition [1.24. In the case that there exist no sources, we have the following.

Proposition 3.9. If $\overline{r\left(E^{1}\right)}=E^{0}$, then $\mathcal{O}(E) \cong \widetilde{\mathcal{O}}_{C_{d}\left(E^{1}\right)}$.

Remark 3.10. In D, V. Deaconu introduced compact graphs which are topological graphs $E=\left(E^{0}, E^{1}, d, r\right)$ such that both $E^{0}$ and $E^{1}$ are compact, and both $d$ and $r$ are surjective and locally homeomorphic. He associated a $C^{*}$-algebra with a compact graph by constructing a certain groupoid, and showed in [D Theorem 4.3] that this is isomorphic to the Cuntz-Pimsner algebras of the $C^{*}$-correspondence defined by the compact graph. Hence Proposition 3.9 implies that his $C^{*}$-algebras are isomorphic to our $C^{*}$-algebras. As he pointed out in the last part of [D], the $C^{*}$-algebras arising from polymorphisms defined in $[\mathrm{AR}]$ are different from our $C^{*}$ algebras in general.

Remark 3.11. We should note that the $C^{*}$-algebra $C^{*}(\tau)$ is the same as the relative Cuntz-Pimsner algebra $\mathcal{O}\left(C_{0}\left(E_{\mathrm{rg}}^{0}\right), C_{d}\left(E^{1}\right)\right)$ determined by the ideal $C_{0}\left(E_{\mathrm{rg}}^{0}\right) \subset$ $C_{0}\left(E^{0}\right)$ [MS, Definition 2.18], and Proposition 3.6 follows from [MS, Proposition 2.21]. By Corollary 4.7 we have the isomorphism $\mathcal{O}(E) \cong \mathcal{O}\left(C_{0}\left(E_{\mathrm{rg}}^{0}\right), C_{d}\left(E^{1}\right)\right)$ (this also can be proved using [MS, Theorem 2.19]).

\section{The GaUge-INVARIANT UNIQUENESS THEOREM}

In this section and the next section, we investigate for which Cuntz-Krieger $E$ pair $T, \rho_{T}$ gives an isomorphism $C^{*}(T) \cong \mathcal{O}(E)$. Such a pair is necessarily injective, but this condition is not sufficient in general. In this section, we give two kinds of extra conditions for the isomorphism $C^{*}(T) \cong \mathcal{O}(E)$, namely the existence of gauge actions and the existence of conditional expectations (Theorem 4.5). In the next section, we deal with the problem of determining topological graphs for which injectivity of $T$ is sufficient for the isomorphism $C^{*}(T) \cong \mathcal{O}(E)$.

By the universality of $\mathcal{O}(E)$, there exists an action $\beta: \mathbb{T} \curvearrowright \mathcal{O}(E)$ defined by $\beta_{z}\left(t^{0}(f)\right)=t^{0}(f)$ and $\beta_{z}\left(t^{1}(\xi)\right)=z t^{1}(\xi)$ for $f \in C_{0}\left(E^{0}\right), \xi \in C_{d}\left(E^{1}\right)$ and $z \in \mathbb{T}$. The action $\beta$ is called the gauge action. It is easy to see that

$$
\beta_{z}\left(t^{n}(\xi) t^{m}(\eta)^{*}\right)=z^{n-m} t^{n}(\xi) t^{m}(\eta)^{*} \quad\left(\xi \in C_{d}\left(E^{n}\right), \eta \in C_{d}\left(E^{m}\right)\right) .
$$


We define a linear map $\Psi: \mathcal{O}(E) \rightarrow \mathcal{O}(E)$ by

$$
\Psi(x)=\int_{\mathbb{T}} \beta_{z}(x) d z
$$

where $d z$ is the normalized Haar measure of $\mathbb{T}$. Then we have

$$
\Psi\left(t^{n}(\xi) t^{m}(\eta)^{*}\right)=\delta_{n, m} t^{n}(\xi) t^{m}(\eta)^{*} \quad\left(\xi \in C_{d}\left(E^{n}\right), \eta \in C_{d}\left(E^{m}\right)\right),
$$

where $\delta_{n, m}$ is the Kronecker delta. Hence $\Psi$ is a faithful conditional expectation onto a subalgebra

$$
\mathcal{F}:=\overline{\operatorname{span}}\left\{t^{k}(\xi) t^{k}(\eta)^{*} \mid \xi, \eta \in C_{d}\left(E^{k}\right), k \in \mathbb{N}\right\} .
$$

Definition 4.1. For a Cuntz-Krieger $E$-pair $T=\left(T^{0}, T^{1}\right)$, we define subalgebras $\mathcal{F}_{T}^{n}, \mathcal{G}_{T}^{n}$ for $n \in \mathbb{N}$ and $\mathcal{F}_{T}$ of $C^{*}(T)$ by

$$
\begin{aligned}
& \mathcal{G}_{T}^{n}=\overline{\operatorname{span}}\left\{T^{n}(\xi) T^{n}(\eta)^{*} \mid \xi, \eta \in C_{d}\left(E^{n}\right)\right\}, \\
& \mathcal{F}_{T}^{n}=\overline{\operatorname{span}}\left\{T^{k}(\xi) T^{k}(\eta)^{*} \mid \xi, \eta \in C_{d}\left(E^{k}\right), 0 \leq k \leq n\right\}, \\
& \mathcal{F}_{T}=\overline{\operatorname{span}}\left\{T^{k}(\xi) T^{k}(\eta)^{*} \mid \xi, \eta \in C_{d}\left(E^{k}\right), \quad k \in \mathbb{N}\right\} .
\end{aligned}
$$

We simply write $\mathcal{F}^{n}, \mathcal{G}^{n}$ for $n \in \mathbb{N}$ and $\mathcal{F}$ for the corresponding subalgebras in $\mathcal{O}(E)$.

Note that $\mathcal{G}_{T}^{n}$ is an ideal of the $C^{*}$-algebra $\mathcal{F}_{T}^{n}$ and that $\mathcal{F}_{T}^{n+1}=\mathcal{G}_{T}^{n+1}+\mathcal{F}_{T}^{n}$ for each $n \in \mathbb{N}$. We also see that $\mathcal{F}_{T}=\overline{\bigcup_{n=0}^{\infty} \mathcal{F}_{T}^{n}}$. Note that $\mathcal{G}_{T}^{n}$ is the image of $\Phi^{n}$, hence $\mathcal{K}\left(C_{d}\left(E^{n}\right)\right) \cong \mathcal{G}_{T}^{n}$ when $T$ is injective. We will show that if a Cuntz-Krieger $E$-pair $T$ is injective, then the restriction of $\rho_{T}$ to $\mathcal{F}$ is an isomorphism onto $\mathcal{F}_{T}$ (Proposition 4.4).

Lemma 4.2. For an injective Cuntz-Krieger E-pair T, we have

$$
\mathcal{G}_{T}^{0} \cap \mathcal{G}_{T}^{1}=T^{0}\left(C_{0}\left(E_{\mathrm{rg}}^{0}\right)\right) .
$$

Proof. This follows from the definition of Cuntz-Krieger pairs and Proposition 2.11

Lemma 4.3. If a Cuntz-Krieger E-pair $T$ is injective, then $\mathcal{F}_{T}^{n} \cap \mathcal{G}_{T}^{n+1}=\mathcal{G}_{T}^{n} \cap$ $\mathcal{G}_{T}^{n+1}=\Phi^{n}\left(\mathcal{K}\left(C_{d}\left(E_{\mathrm{rg}}^{n}\right)\right)\right)$ for every $n \in \mathbb{N}$.

Proof. Take $x \in \mathcal{F}_{T}^{n} \cap \mathcal{G}_{T}^{n+1}$. Let $\left\{u_{i}\right\}_{i \in \mathbb{I}}$ be an approximate unit of $\mathcal{G}_{T}^{n}$. Since

$$
\mathcal{G}_{T}^{n+1}=\overline{\operatorname{span}}\left\{T^{n}(\xi) T^{1}\left(\xi^{\prime}\right) T^{1}\left(\eta^{\prime}\right)^{*} T^{n}(\eta)^{*} \mid \xi, \eta \in C_{d}\left(E^{n}\right), \xi^{\prime}, \eta^{\prime} \in C_{d}\left(E^{1}\right)\right\},
$$

$\left\{u_{i}\right\}_{i \in \mathbb{I}}$ is also an approximate unit of $\mathcal{G}_{T}^{n+1}$. Hence we have $x=\lim _{i} u_{i} x$. Since $x \in \mathcal{F}_{T}^{n}$ and $\mathcal{G}_{T}^{n}$ is an ideal of $\mathcal{F}_{T}^{n}$, we have $u_{i} x \in \mathcal{G}_{T}^{n}$ for $i \in \mathbb{I}$. Hence $x \in \mathcal{G}_{T}^{n}$. Thus we have $\mathcal{F}_{T}^{n} \cap \mathcal{G}_{T}^{n+1}=\mathcal{G}_{T}^{n} \cap \mathcal{G}_{T}^{n+1}$.

Take $\xi, \eta \in C_{d}\left(E_{\mathrm{rg}}^{n}\right)$ arbitrarily. We can find $\xi^{\prime} \in C_{d}\left(E^{n}\right)$ and $f \in C_{0}\left(E_{\mathrm{rg}}^{0}\right)$ with $\xi=\xi^{\prime} f$. Since $T$ is a Cuntz-Krieger $E$-pair, we have

$$
\Phi^{n}\left(\theta_{\xi, \eta}\right)=T^{n}(\xi) T^{n}(\eta)^{*}=T^{n}\left(\xi^{\prime}\right) T^{0}(f) T^{n}(\eta)^{*}=T^{n}\left(\xi^{\prime}\right) \Phi^{1}\left(\pi_{r}(f)\right) T^{n}(\eta)^{*} \in \mathcal{G}_{T}^{n+1} .
$$

Thus $\Phi^{n}\left(\mathcal{K}\left(C_{d}\left(E_{\mathrm{rg}}^{n}\right)\right)\right) \subset \mathcal{G}_{T}^{n} \cap \mathcal{G}_{T}^{n+1}$. Conversely take $x \in \mathcal{K}\left(C_{d}\left(E^{n}\right)\right)$ with $\Phi^{n}(x) \in$ $\mathcal{G}_{T}^{n} \cap \mathcal{G}_{T}^{n+1}$. For $\xi, \eta \in C_{d}\left(E^{n}\right)$, we have

$$
T^{0}(\langle\xi, x \eta\rangle)=T^{n}(\xi)^{*} \Phi^{n}(x) T^{n}(\eta) \in \mathcal{G}_{T}^{0} \cap \mathcal{G}_{T}^{1} .
$$

By Lemma 4.2 we have $\langle\xi, x \eta\rangle \in C_{0}\left(E_{\mathrm{rg}}^{0}\right)$. Therefore we get $x \in \mathcal{K}\left(C_{d}\left(E_{\mathrm{rg}}^{n}\right)\right)$ by Lemma 1.14. Thus we have shown that $\mathcal{F}_{T}^{n} \cap \mathcal{G}_{T}^{n+1}=\mathcal{G}_{T}^{n} \cap \mathcal{G}_{T}^{n+1}=\Phi^{n}\left(\mathcal{K}\left(C_{d}\left(E_{\mathrm{rg}}^{n}\right)\right)\right)$. 
Proposition 4.4. For an injective Cuntz-Krieger E-pair T, the restriction of $\rho_{T}$ to $\mathcal{F}$ is an isomorphism onto $\mathcal{F}_{T}$.

Proof. First note that the restriction of $\rho_{T}$ to $\mathcal{G}^{n}$ is an isomorphism onto $\mathcal{G}_{T}^{n}$ for every $n \in \mathbb{N}$ because $\rho_{T} \circ \varphi^{n}=\Phi^{n}$ and $\varphi^{n}, \Phi^{n}$ are isomorphisms onto $\mathcal{G}^{n}$ and $\mathcal{G}_{T}^{n}$ respectively. To finish the proof, it suffices to show that the restriction of $\rho_{T}$ to $\mathcal{F}^{n}$ is an isomorphism onto $\mathcal{F}_{T}^{n}$ for every $n \in \mathbb{N}$. We will prove this by induction with respect to $n \in \mathbb{N}$. The restriction of $\rho_{T}$ to $\mathcal{F}^{0}$ is an isomorphism onto $\mathcal{F}_{T}^{0}$ because $\mathcal{F}^{0}=\mathcal{G}^{0}$ and $\mathcal{F}_{T}^{0}=\mathcal{G}_{T}^{0}$. Assume that the restriction of $\rho_{T}$ to $\mathcal{F}^{n}$ is an isomorphism onto $\mathcal{F}_{T}^{n}$. We have the following commutative diagram with exact rows:

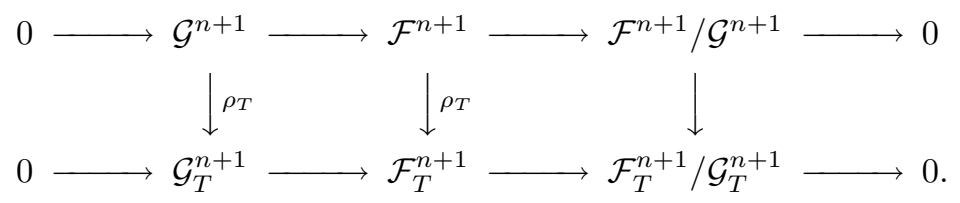

To prove that the restriction of $\rho_{T}$ to $\mathcal{F}^{n+1}$ is an isomorphism onto $\mathcal{F}_{T}^{n+1}$, it is sufficient to see that the map $\mathcal{F}^{n+1} / \mathcal{G}^{n+1} \rightarrow \mathcal{F}_{T}^{n+1} / \mathcal{G}_{T}^{n+1}$ induced by $\rho_{T}$ is an isomorphism. Since $\mathcal{F}_{T}^{n+1}=\mathcal{F}_{T}^{n}+\mathcal{G}_{T}^{n+1}$, we have

$$
\mathcal{F}_{T}^{n+1} / \mathcal{G}_{T}^{n+1} \cong \mathcal{F}_{T}^{n} /\left(\mathcal{F}_{T}^{n} \cap \mathcal{G}_{T}^{n+1}\right)=\mathcal{F}_{T}^{n} /\left(\mathcal{G}_{T}^{n} \cap \mathcal{G}_{T}^{n+1}\right) .
$$

By the assumption of the induction, the restriction of $\rho_{T}$ to $\mathcal{F}^{n}$ is an isomorphism onto $\mathcal{F}_{T}^{n}$. By Lemma 4.3 we have

$$
\mathcal{G}^{n} \cap \mathcal{G}^{n+1} \cong \mathcal{K}\left(C_{d}\left(E_{\mathrm{rg}}^{n}\right)\right) \cong \mathcal{G}_{T}^{n} \cap \mathcal{G}_{T}^{n+1} .
$$

Hence the restriction of $\rho_{T}$ to $\mathcal{G}^{n} \cap \mathcal{G}^{n+1}$ is an isomorphism onto $\mathcal{G}_{T}^{n} \cap \mathcal{G}_{T}^{n+1}$. Therefore the map $\mathcal{F}^{n} /\left(\mathcal{G}^{n} \cap \mathcal{G}^{n+1}\right) \rightarrow \mathcal{F}_{T}^{n} /\left(\mathcal{G}_{T}^{n} \cap \mathcal{G}_{T}^{n+1}\right)$ induced by $\rho_{T}$ is an isomorphism. Hence the map $\mathcal{F}^{n+1} / \mathcal{G}^{n+1} \rightarrow \mathcal{F}_{T}^{n+1} / \mathcal{G}_{T}^{n+1}$ induced by $\rho_{T}$ is also an isomorphism. Thus we have shown that the restriction of $\rho_{T}$ to $\mathcal{F}^{n+1}$ is an isomorphism onto $\mathcal{F}_{T}^{n+1}$. We are done.

Now we have the following gauge-invariant uniqueness theorem.

Theorem 4.5. For a topological graph $E=\left(E^{0}, E^{1}, d, r\right)$ and a Cuntz-Krieger E-pair $T=\left(T^{0}, T^{1}\right)$, the following are equivalent:

(i) The $\operatorname{map} \rho_{T}: \mathcal{O}(E) \rightarrow C^{*}(T)$ is an isomorphism.

(ii) The map $T^{0}$ is injective and there exists an automorphism $\beta_{z}^{\prime}$ of $C^{*}(T)$ such that $\beta_{z}^{\prime}\left(T^{0}(f)\right)=T^{0}(f)$ and $\beta_{z}^{\prime}\left(T^{1}(\xi)\right)=z T^{1}(\xi)$ for every $z \in \mathbb{T}$.

(iii) The map $T^{0}$ is injective and there exists a conditional expectation $\Psi_{T}$ from $C^{*}(T)$ onto $\mathcal{F}_{T}$ such that $\Psi_{T}\left(T^{n}(\xi) T^{m}(\eta)^{*}\right)=\delta_{n, m} T^{n}(\xi) T^{m}(\eta)^{*}$ for $\xi \in$ $C_{d}\left(E^{n}\right)$ and $\eta \in C_{d}\left(E^{m}\right)$.

Proof. (i) $\Rightarrow$ (ii): Already shown.

(ii) $\Rightarrow$ (iii): Set $\Psi_{T}(x)=\int_{\mathbb{T}} \beta_{z}^{\prime}(x) d z$.

(iii) $\Rightarrow\left(\right.$ i): Since the map $T^{0}$ is injective, we see that the restriction of $\rho_{T}$ to $\mathcal{F}$ is an isomorphism onto $\mathcal{F}_{T}$ by Proposition 4.4. Now we see that the map $\rho_{T}: \mathcal{O}(E) \rightarrow$ $C^{*}(T)$ is an isomorphism by the standard argument of conditional expectations (see, for example, Ka1, Proposition 3.11]).

Remark 4.6. If there exists an automorphism $\beta_{z}^{\prime}$ of $C^{*}(T)$ such that $\beta_{z}^{\prime}\left(T^{0}(f)\right)=$ $T^{0}(f)$ and $\beta_{z}^{\prime}\left(T^{1}(\xi)\right)=z T^{1}(\xi)$ for every $z \in \mathbb{T}$, then $\beta^{\prime}: \mathbb{T} \ni z \mapsto \beta_{z}^{\prime} \in \operatorname{Aut}\left(C^{*}(T)\right)$ 
becomes automatically a strongly continuous homomorphism. This fact is used implicitly in the proof of the implication (ii) $\Rightarrow$ (iii) in the above theorem.

Corollary 4.7. The Fock representation $\rho_{\tau}$ of $\mathcal{O}(E)$ is faithful.

Proof. We check the condition (ii) in Theorem 4.5 We have already seen that $\tau^{0}$ is injective in Proposition 3.6. For $z \in \mathbb{T}$, define a unitary $u_{z} \in \mathcal{L}\left(C_{d}\left(E^{*}\right)\right)$ by $u_{z}(\xi)=z^{n} \xi$ for $\xi \in C_{d^{n}}\left(E^{n}\right) \subset C_{d}\left(E^{*}\right)(n \in \mathbb{N})$. We define an automorphism $\beta_{z}^{\prime}=\operatorname{Ad}\left(\pi\left(u_{z}\right)\right)$ of $\mathcal{L}\left(C_{d}\left(E^{*}\right)\right) / \mathcal{K}\left(C_{d}\left(E_{\mathrm{rg}}^{*}\right)\right)$ by $\beta_{z}^{\prime}(x)=\pi\left(u_{z}\right) x \pi\left(u_{z}\right)^{*}$, where $\pi$ is the natural surjection

$$
\mathcal{L}\left(C_{d}\left(E^{*}\right)\right) \rightarrow \mathcal{L}\left(C_{d}\left(E^{*}\right)\right) / \mathcal{K}\left(C_{d}\left(E_{\mathrm{rg}}^{*}\right)\right) .
$$

One can easily see that $\beta_{z}^{\prime}\left(\tau^{0}(f)\right)=\tau^{0}(f)$ and $\beta_{z}^{\prime}\left(\tau^{1}(\xi)\right)=z \tau^{1}(\xi)$ for $f \in C_{0}\left(E^{0}\right)$ and $\xi \in C_{d}\left(E^{1}\right)$. This implies that $\beta_{z}^{\prime}$ fixes $C^{*}(\tau)$ globally for each $z \in \mathbb{T}$. Hence the restriction of $\beta_{z}^{\prime}$ to $C^{*}(\tau)$ is an automorphism of $C^{*}(\tau)$. By Theorem 4.5, we see that $\rho_{\tau}: \mathcal{O}(E) \rightarrow C^{*}(\tau)$ is an isomorphism.

\section{The Cuntz-Krieger uniqueness theorem}

In this section, we see that if a graph $E$ satisfies a certain condition, then the condition that $T^{0}$ is injective is not only necessary but sufficient for $C^{*}(T) \cong \mathcal{O}(E)$. To this end, we need a more precise description of $\mathcal{F}_{T}^{n}$ for an injective Cuntz-Krieger $E$-pair $T$. Let us fix an injective Cuntz-Krieger $E$-pair $T$ for a while.

For each $n \in \mathbb{N}$, we define a $*$-homomorphism $\pi_{n}^{n}: \mathcal{F}_{T}^{n} \rightarrow \mathcal{L}\left(C_{d}\left(E^{n}\right)\right)$ by

$$
T^{n}\left(\pi_{n}^{n}(x) \xi\right)=x T^{n}(\xi) \quad \text { for } x \in \mathcal{F}_{T}^{n}, \xi \in C_{d}\left(E^{n}\right) .
$$

Note that $T^{n}: C_{d}\left(E^{n}\right) \rightarrow C^{*}(T)$ is injective and that $x T^{n}(\xi)$ lies in the image of $T^{n}$ for $x \in \mathcal{F}_{T}^{n}, \xi \in C_{d}\left(E^{n}\right)$. The restriction of $\pi_{n}^{n}$ to $\mathcal{G}_{T}^{n}$ coincides with the isomorphism $\Phi^{n}: \mathcal{G}_{T}^{n} \rightarrow \mathcal{K}\left(C_{d}\left(E^{n}\right)\right)$.

We define a closed subset $E_{\mathrm{sg}}^{n}$ of $E^{n}$ by $E_{\mathrm{sg}}^{n}=\left(d^{n}\right)^{-1}\left(E_{\mathrm{sg}}^{0}\right)=E^{n} \backslash E_{\mathrm{rg}}^{n}$ for each $n \in \mathbb{N}$. Recall that there exists a $*$-homomorphism $\omega^{n}: \mathcal{L}\left(C_{d}\left(E^{n}\right)\right) \rightarrow \mathcal{L}\left(C_{d}\left(E_{\mathrm{sg}}^{n}\right)\right)$, and that the restriction of $\omega^{n}$ to $\mathcal{K}\left(C_{d}\left(E^{n}\right)\right)$ is a surjective map to $\mathcal{K}\left(C_{d}\left(E_{\mathrm{sg}}^{n}\right)\right)$, whose kernel is $\mathcal{K}\left(C_{d}\left(E_{\mathrm{rg}}^{n}\right)\right)$ (Lemma 1.14). We denote by $\dot{\pi}_{n}^{n}: \mathcal{F}_{T}^{n} \rightarrow \mathcal{L}\left(C_{d}\left(E_{\mathrm{sg}}^{n}\right)\right)$ the composition of the map $\pi_{n}^{n}: \mathcal{F}_{T}^{n} \rightarrow \mathcal{L}\left(C_{d}\left(E^{n}\right)\right)$ and the surjection $\omega^{n}: \mathcal{L}\left(C_{d}\left(E^{n}\right)\right) \rightarrow$ $\mathcal{L}\left(C_{d}\left(E_{\mathrm{sg}}^{n}\right)\right)$.

Lemma 5.1. For each $n \in \mathbb{N}$, we can define $*$-homomorphisms

$$
\pi_{k}^{n}: \mathcal{F}_{T}^{n} \rightarrow \mathcal{L}\left(C_{d}\left(E_{\mathrm{sg}}^{k}\right)\right)
$$

for $k=0, \ldots, n-1$ such that $\bigcap_{k=0}^{n-1} \operatorname{ker} \pi_{k}^{n}=\mathcal{G}_{T}^{n}$.

Proof. The proof goes by induction with respect to $n \in \mathbb{N}$. For $n=0$, we need to do nothing because $\mathcal{G}_{T}^{0}=\mathcal{F}_{T}^{0}$. Assume that we have $*$-homomorphisms $\pi_{k}^{n}: \mathcal{F}_{T}^{n} \rightarrow$ $\mathcal{L}\left(C_{d}\left(E_{\mathrm{sg}}^{k}\right)\right)$ for $k=0, \ldots, n-1$ such that $\bigcap_{k=0}^{n-1} \operatorname{ker} \pi_{k}^{n}=\mathcal{G}_{T}^{n}$. Then we have

$$
\begin{aligned}
\bigcap_{k=0}^{n-1} \operatorname{ker} \pi_{k}^{n} \cap \operatorname{ker} \dot{\pi}_{n}^{n} & =\mathcal{G}_{T}^{n} \cap \operatorname{ker} \dot{\pi}_{n}^{n}=\Phi^{n}\left(\mathcal{K}\left(C_{d}\left(E^{n}\right)\right) \cap \operatorname{ker} \omega^{n}\right) \\
& =\Phi^{n}\left(\mathcal{K}\left(C_{d}\left(E_{\mathrm{rg}}^{n}\right)\right)\right)=\mathcal{G}_{T}^{n} \cap \mathcal{G}_{T}^{n+1} .
\end{aligned}
$$

Hence the maps $\pi_{0}^{n}, \ldots, \pi_{n-1}^{n}$ and $\dot{\pi}_{n}^{n}$ factor through maps

$$
\widetilde{\pi}_{k}^{n+1}: \mathcal{F}_{T}^{n} /\left(\mathcal{G}_{T}^{n} \cap \mathcal{G}_{T}^{n+1}\right) \rightarrow \mathcal{L}\left(C_{d}\left(E_{\mathrm{sg}}^{k}\right)\right) \quad(k=0, \ldots, n),
$$


and we see that $\bigcap_{k=0}^{n} \operatorname{ker} \widetilde{\pi}_{k}^{n+1}=0$. For $k=0, \ldots, n$, we define $\pi_{k}^{n+1}: \mathcal{F}_{T}^{n+1} \rightarrow$ $\mathcal{L}\left(C_{d}\left(E_{\text {sg }}^{k}\right)\right)$ by the composition of the quotient map $\mathcal{F}_{T}^{n+1} \rightarrow \mathcal{F}_{T}^{n+1} / \mathcal{G}_{T}^{n+1}$, the isomorphism $\mathcal{F}_{T}^{n+1} / \mathcal{G}_{T}^{n+1} \cong \mathcal{F}_{T}^{n} /\left(\mathcal{G}_{T}^{n} \cap \mathcal{G}_{T}^{n+1}\right)$, and $\widetilde{\pi}_{k}^{n+1}$. Then we have $\bigcap_{k=0}^{n} \operatorname{ker} \pi_{k}^{n+1}$ $=\mathcal{G}_{T}^{n+1}$.

For $k=0, \ldots, n-1$, the $*$-homomorphism $\pi_{k}^{n}: \mathcal{F}_{T}^{n} \rightarrow \mathcal{L}\left(C_{d}\left(E_{\mathrm{sg}}^{k}\right)\right)$ defined in the proof of Lemma 5.1 is determined by

$$
\pi_{k}^{n}(x)= \begin{cases}\dot{\pi}_{k}^{k}(x) & \text { if } x \in \mathcal{F}_{T}^{k}, \\ 0 & \text { if } x \in \mathcal{G}_{T}^{l} \text { for } k<l \leq n .\end{cases}
$$

Proposition 5.2. For $n \in \mathbb{N}$, the map

$$
\bigoplus_{k=0}^{n} \pi_{k}^{n}: \mathcal{F}_{T}^{n} \rightarrow \bigoplus_{k=0}^{n-1} \mathcal{L}\left(C_{d}\left(E_{\mathrm{sg}}^{k}\right)\right) \oplus \mathcal{L}\left(C_{d}\left(E^{n}\right)\right)
$$

is injective.

Proof. This follows from

$$
\operatorname{ker}\left(\bigoplus_{k=0}^{n} \pi_{k}^{n}\right)=\bigcap_{k=0}^{n} \operatorname{ker} \pi_{k}^{n}=\mathcal{G}_{T}^{n} \cap \operatorname{ker} \pi_{n}^{n}=0 .
$$

Definition 5.3. Let $E$ be a topological graph. A path $e=\left(e_{n}, \ldots, e_{1}\right) \in E^{n}$ for $n \geq 1$ is called a loop if $r(e)=d(e)$, and the vertex $r(e)=d(e)$ is called the base point of the loop $e$. A loop $e=\left(e_{n}, \ldots, e_{1}\right)$ is said to be without entrances if $r^{-1}\left(r\left(e_{k}\right)\right)=\left\{e_{k}\right\}$ for $k=1, \ldots, n$.

It is easy to see that when $E$ is an ordinary dynamical system, every loop is without entrances and $v \in E^{0}$ is a base point of a loop if and only if it is a periodic point. We generalize the notion of topological freeness of homeomorphisms to topological correspondences. Recall that a homeomorphism on a space is called topologically free if the set of periodic points has an empty interior (see [AS], [T1], ELQ]).

Definition 5.4. A topological graph $E$ is said to be topologically free if the set of base points of loops without entrances has an empty interior.

One can easily see that topological freeness coincides with Condition $L$ when a graph $E$ is discrete (see $[\mathrm{KPR}]$ ). We will show that when a topological graph $E$ is topologically free, $\rho_{T}$ is an isomorphism if and only if $T^{0}$ is injective (Theorem 5.12). To do so, we need the following notion and many lemmas.

Definition 5.5. Let $n$ be an integer with $n \geq 1$. A path $e=\left(e_{n}, \ldots, e_{1}\right) \in E^{n}$ is said to be returning if $e_{1}=e_{k}$ for some $k \in\{2, \ldots, n\}$. Otherwise $e$ is said to be non-returning. A non-empty set $U \subset E^{n}$ is said to be non-returning if $e_{1} \neq e_{k}^{\prime}$ for every $k=2, \ldots, n$ and every $\left(e_{m}, \ldots, e_{1}\right),\left(e_{m}^{\prime}, \ldots, e_{1}^{\prime}\right) \in U$.

Lemma 5.6. Let $V$ be an open subset of $E^{0}$, and $e=\left(e_{n}, \ldots, e_{1}\right) \in E^{n}(n \geq 1)$ be a non-returning path with $r^{n}(e) \in V$. Then there exists a non-empty open set $U \subset\left(r^{n}\right)^{-1}(V) \subset E^{n}$ which is non-returning. 
Proof. Take open subsets $U_{1}, \ldots, U_{n}$ of $E^{1}$ such that $e_{k} \in U_{k}$ for $k=1, \ldots, n$, $U_{k} \cap U_{1}=\emptyset$ for $k=2, \ldots, n$, and $r^{1}\left(U_{n}\right) \subset V$. Then a non-empty open subset $U=\left(U_{n} \times \cdots \times U_{1}\right) \cap E^{n}$ of $E^{n}$ is non-returning.

Lemma 5.7. Suppose that an open set $U \subset E^{m}$ is non-returning. Take $\zeta \in$ $C_{c}(U) \subset C_{d}\left(E^{m}\right)$ and $\xi \in C_{d}\left(E^{n}\right)$ for $1 \leq n \leq m-1$. Then $T^{m}(\zeta)^{*} T^{n}(\xi) T^{m}(\zeta)$ $=0$ for any Toeplitz E-pair $T$.

Proof. By Lemma 2.4, we have $T^{m}(\zeta)^{*} T^{n}(\xi) T^{m}(\zeta)=T^{n}(\eta)$ where $\eta \in C_{d}\left(E^{n}\right)$ is defined by

$$
\eta\left(e_{n}, \ldots, e_{1}\right)=\sum_{\substack{\left(e_{n+m}, \ldots, e_{n+1}\right) \in E^{m} \\ d\left(e_{n+1}\right)=r\left(e_{n}\right)}} \overline{\zeta\left(e_{n+m}, \ldots, e_{n+1}\right)} \xi\left(e_{n+m}, \ldots, e_{m+1}\right) \zeta\left(e_{m}, \ldots, e_{1}\right) .
$$

For each $\left(e_{n}, \ldots, e_{1}\right) \in E^{n}$, we see that $\left(e_{n+m}, \ldots, e_{n+1}\right) \in U$ implies $\left(e_{m}, \ldots, e_{1}\right) \notin$ $U$ by the assumption on $U$. Hence we have $\eta=0$. Thus $T^{m}(\zeta)^{*} T^{n}(\xi) T^{m}(\zeta)=$ 0 .

Lemma 5.8. Suppose that an open subset $V$ of $E^{0}$ satisfies $\left(r^{k}\right)^{-1}(V) \neq \emptyset$ and $\left(r^{k+1}\right)^{-1}(V)=\emptyset$ for some $k \in \mathbb{N}$. Take $\zeta \in C_{c}\left(\left(r^{k}\right)^{-1}(V)\right) \subset C_{d}\left(E^{k}\right)$ and $\xi \in$ $C_{d}\left(E^{l}\right)$ for $l>k$. Then we have $T^{k}(\zeta)^{*} T^{l}(\xi)=0$ for any Toeplitz E-pair $T$.

Proof. By Lemma 2.4, we have $T^{k}(\zeta)^{*} T^{l}(\xi)=T^{m}(\eta)$ where $m=l-k>0$ and $\eta \in C_{d}\left(E^{m}\right)$ is determined by

$$
\eta(e)=\sum_{\substack{e^{\prime} \in E^{k} \\ d^{k}\left(e^{\prime}\right)=r^{m}(e)}} \overline{\zeta\left(e^{\prime}\right)} \xi\left(e^{\prime}, e\right) \quad\left(e \in E^{m}\right) .
$$

For $e \in E^{m}$, there exists no $e^{\prime} \in\left(r^{k}\right)^{-1}(V) \subset E^{k}$ with $d^{k}\left(e^{\prime}\right)=r^{m}(e)$ by the assumption. Hence $\eta=0$. Thus $T^{k}(\zeta)^{*} T^{l}(\xi)=0$.

Lemma 5.9. Suppose that a topological graph $E=\left(E^{0}, E^{1}, d, r\right)$ is topologically free. For an open subset $V$ of $E^{0}$ and a positive integer $n$, either $\left(r^{n}\right)^{-1}(V)=\emptyset$ or there exists a non-returning path $e \in E^{m}$ with $m \geq n$ such that $r^{m}(e) \in V$.

Proof. To the contrary, assume that an open subset $V$ of $E^{0}$ satisfies $\left(r^{n}\right)^{-1}(V) \neq$ $\emptyset$ and that every path in $\left(r^{m}\right)^{-1}(V)$ is returning for every $m \geq n$. Take $e=$ $\left(e_{n}, \ldots, e_{1}\right) \in\left(r^{n}\right)^{-1}(V)$ arbitrarily. Since $e$ is returning, there exists $k_{0}$ with $2 \leq$ $k_{0} \leq n$ such that $e_{k_{0}}=e_{1}$. We will show that $r^{-1}\left(r\left(e_{l}\right)\right)=\left\{e_{l}\right\}$ for $l=1, \ldots, k_{0}-1$. To derive a contradiction, assume that there exist an integer $l$ with $1 \leq l<k_{0}$ and $e \in E^{1}$ such that $r(e)=r\left(e_{l}\right)$ and $e \neq e_{l}$. Set $v=r(e)=r\left(e_{l}\right)$. Let $k_{1}$ be a maximum integer satisfying $r\left(e_{k_{1}}\right)=v$. We have $d\left(e_{k_{1}+1}\right)=v$ and $e_{k} \neq e$ for $k=k_{1}+1, \ldots, n$. Since $\left(e_{k_{0}-1}, \ldots, e_{1}\right)$ is a loop, we can find a loop $e^{\prime}=\left(e_{m}^{\prime}, \ldots, e_{1}^{\prime}\right)$ such that $e_{m}^{\prime}=e_{l}$ and $r\left(e_{k}^{\prime}\right) \neq v$ for $k=1, \ldots, m-1$. Hence we have $e_{k}^{\prime} \neq e$ for $k=1, \ldots, m$. Set

$$
e^{\prime \prime}=\left(e_{n}, \ldots, e_{k_{1}+1}, e^{\prime}, e^{\prime}, \ldots, e^{\prime}, e\right) \in E^{n^{\prime}}
$$

where $e^{\prime}$ is repeated so that $n^{\prime} \geq n$. Then $e^{\prime \prime} \in E^{n^{\prime}}$ is a non-returning path with $r\left(e^{\prime \prime}\right) \in V$. This is a contradiction. Therefore, we have shown that for every $\left(e_{n}, \ldots, e_{1}\right) \in\left(r^{n}\right)^{-1}(V)$, there exists an integer $k_{0}$ with $2 \leq k_{0} \leq n$ such that $\left(e_{k_{0}-1}, \ldots, e_{1}\right)$ is a loop without entrances. Thus each element in a non-empty open subset $d^{n}\left(\left(r^{n}\right)^{-1}(V)\right)$ of $E^{0}$ is a base point of a loop without entrances. This contradicts the fact that $E$ is topologically free. 
Proposition 5.10. Let $E=\left(E^{0}, E^{1}, d, r\right)$ be a topologically free topological graph, and $T=\left(T^{0}, T^{1}\right)$ be an injective Cuntz-Krieger E-pair. Take $n_{l}, m_{l} \in \mathbb{N}, \xi_{l} \in$ $C_{d}\left(E^{n_{l}}\right)$ and $\eta_{l} \in C_{d}\left(E^{m_{l}}\right)$ for $l=1,2, \ldots, L$. Define

$$
x=\sum_{l=1}^{L} T^{n_{l}}\left(\xi_{l}\right) T^{m_{l}}\left(\eta_{l}\right)^{*}, \quad x_{0}=\sum_{n_{l}=m_{l}} T^{n_{l}}\left(\xi_{l}\right) T^{m_{l}}\left(\eta_{l}\right)^{*} .
$$

Then for arbitrary $\varepsilon>0$ there exist $a, b \in C^{*}(T)$ and $f \in C_{0}\left(E^{0}\right)$ such that $\|a\|,\|b\| \leq 1,\|f\|=\left\|x_{0}\right\|$, and $\left\|a^{*} x b-T^{0}(f)\right\|<\varepsilon$.

Proof. Set $n=\max \left\{n_{1}, \ldots, n_{L}, m_{1}, \ldots, m_{L}\right\}$. We have $x_{0} \in \mathcal{F}_{T}^{n}$. Since

$$
\bigoplus_{k=0}^{n} \pi_{k}^{n}: \mathcal{F}_{T}^{n} \rightarrow \bigoplus_{k=0}^{n-1} \mathcal{L}\left(C_{d}\left(E_{\mathrm{sg}}^{k}\right)\right) \oplus \mathcal{L}\left(C_{d}\left(E^{n}\right)\right)
$$

is injective, there exists an integer $k$ with $0 \leq k \leq n$ such that $\left\|x_{0}\right\|=\left\|\pi_{k}^{n}\left(x_{0}\right)\right\|$.

Case $1, k \leq n-1$. There exist $\xi^{\prime}, \eta^{\prime} \in C_{d}\left(E_{\mathrm{sg}}^{k}\right)$ with $\left\|\xi^{\prime}\right\|=\left\|\eta^{\prime}\right\|=1$ such that

$$
\left\|\left\langle\xi^{\prime}, \pi_{k}^{n}\left(x_{0}\right) \eta^{\prime}\right\rangle\right\|>\left\|\pi_{k}^{n}\left(x_{0}\right)\right\|-\varepsilon=\left\|x_{0}\right\|-\varepsilon .
$$

By Lemma 1.11 we can find $\xi, \eta \in C_{d}\left(E^{k}\right)$ with $\|\xi\|=\|\eta\|=1$ such that $\left.\xi\right|_{E_{\mathrm{sg}}^{k}}=\xi^{\prime}$ and $\left.\eta\right|_{E_{\mathrm{sg}}^{k}}=\eta^{\prime}$. For each $l=1, \ldots, L$, there exist $n_{l}^{\prime}, m_{l}^{\prime} \in \mathbb{N}$ and $\xi_{l}^{\prime} \in C_{d}\left(E^{n_{l}^{\prime}}\right), \eta_{l}^{\prime} \in$ $C_{d}\left(E^{m_{l}^{\prime}}\right)$ such that

$$
T^{n_{l}^{\prime}}\left(\xi_{l}^{\prime}\right) T^{m_{l}^{\prime}}\left(\eta_{l}^{\prime}\right)^{*}=T^{k}(\xi)^{*} T^{n_{l}}\left(\xi_{l}\right) T^{m_{l}}\left(\eta_{l}\right)^{*} T^{k}(\eta) .
$$

We set

$$
y=\sum_{l=1}^{L} T^{n_{l}^{\prime}}\left(\xi_{l}^{\prime}\right) T^{m_{l}^{\prime}}\left(\eta_{l}^{\prime}\right)^{*} \quad \text { and } \quad T^{0}(g)=\sum_{n_{l}^{\prime}=m_{l}^{\prime}=0} T^{n_{l}^{\prime}}\left(\xi_{l}^{\prime}\right) T^{m_{l}^{\prime}}\left(\eta_{l}^{\prime}\right)^{*} .
$$

Then we have $y=T^{k}(\xi)^{*} x T^{k}(\eta)$ and $\left.g\right|_{E_{\mathrm{Sg}}^{0}}=\left\langle\xi^{\prime}, \pi_{k}^{n}\left(x_{0}\right) \eta^{\prime}\right\rangle$. Since $\left\|x_{0}\right\|-\varepsilon<$ $\left\|\left.g\right|_{E_{\mathrm{Sg}}^{0}}\right\| \leq\left\|x_{0}\right\|$, we can find $v \in E_{\mathrm{sg}}^{0}$ with $\left\|x_{0}\right\|-\varepsilon<|g(v)| \leq\left\|x_{0}\right\|$. Since $E_{\mathrm{sg}}^{0}=\overline{E_{\mathrm{sce}}^{0}} \cup E_{\mathrm{inf}}^{0}$, there are two cases, namely the case that $v \in \overline{E_{\mathrm{sce}}^{0}}$ and the case that $v \in E_{\text {inf }}^{0}$.

Subcase 1.1, $v \in \overline{E_{\mathrm{sce}}^{0}}$. There exists $v^{\prime} \in E_{\mathrm{sce}}^{0}$ with $\left\|x_{0}\right\|-\varepsilon<\left|g\left(v^{\prime}\right)\right| \leq\left\|x_{0}\right\|$. Since

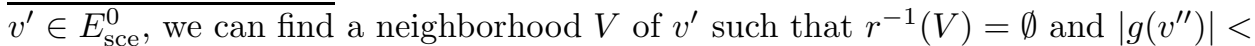
$\left\|x_{0}\right\|+\varepsilon$ for all $v^{\prime \prime} \in V$. Take $h \in C_{0}(V)$ with $0 \leq h \leq 1$ and $h\left(v^{\prime}\right)=1$. We set $a=T^{k}(\xi) T^{0}(h)$ and $b=T^{k}(\eta) T^{0}(h)$. Then we have $\|a\|,\|b\| \leq 1$ and $a^{*} x b=$ $T^{0}(h) y T^{0}(h)=T^{0}(h) T^{0}(g) T^{0}(h)$ because if either $n_{l}^{\prime}$ or $m_{l}^{\prime}$ is greater than 0 , then $T^{0}(h) T^{n_{l}^{\prime}}\left(\xi_{l}^{\prime}\right) T^{m_{l}^{\prime}}\left(\eta_{l}^{\prime}\right)^{*} T^{0}(h)=0$ by Lemma 5.8 Define $f^{\prime}=h g h$. Then we have $\left\|x_{0}\right\|-\varepsilon<\left\|f^{\prime}\right\|<\left\|x_{0}\right\|+\varepsilon$. Set $f=\left\|x_{0}\right\| f^{\prime} /\left\|f^{\prime}\right\|$. Then we have $\|f\|=\left\|x_{0}\right\|$ and $\left\|f-f^{\prime}\right\|=\left|\left\|x_{0}\right\|-\left\|f^{\prime}\right\|\right|<\varepsilon$. Thus we get

$$
\left\|a^{*} x b-T^{0}(f)\right\|=\left\|T^{0}\left(f^{\prime}\right)-T^{0}(f)\right\|<\varepsilon .
$$

Subcase 1.2, $v \in E_{\text {inf }}^{0}$. Choose a positive number $\delta$ such that $\delta<\varepsilon$ and $\left\|x_{0}\right\|-\delta<$

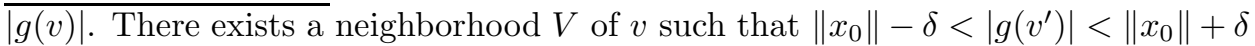
for all $v^{\prime} \in V$. For $l$ with $n_{l}^{\prime}=0$, we set $\xi_{l}^{\prime \prime}=\xi_{l}^{\prime}$. For $l$ with $n_{l}^{\prime} \geq 1$, we set $\xi_{l}^{\prime \prime} \in C_{c}\left(E^{n_{l}^{\prime}}\right)$ sufficiently close to $\xi_{l}^{\prime}$. Similarly, we set $\eta_{l}^{\prime \prime}=\eta_{l}^{\prime}$ for $l$ with $m_{l}^{\prime}=0$, and set $\eta_{l}^{\prime \prime} \in C_{c}\left(E^{n_{l}^{\prime}}\right)$ sufficiently close to $\eta_{l}^{\prime}$ for $l$ with $m_{l}^{\prime} \geq 1$. We set $y^{\prime}=$ 
$\sum_{l=1}^{L} T^{n_{l}^{\prime}}\left(\xi_{l}^{\prime \prime}\right) T^{m_{l}^{\prime}}\left(\eta_{l}^{\prime \prime}\right)^{*}$. We can choose $\xi_{l}^{\prime \prime}$ and $\eta_{l}^{\prime \prime}$ so that $\left\|y-y^{\prime}\right\|<\varepsilon-\delta$. We have $T^{0}(g)=\sum_{n_{l}^{\prime}=m_{l}^{\prime}=0} T^{n_{l}^{\prime}}\left(\xi_{l}^{\prime \prime}\right) T^{m_{l}^{\prime}}\left(\eta_{l}^{\prime \prime}\right)^{*}$. Set

$$
\begin{aligned}
K=\bigcup_{n_{l}^{\prime} \geq 1}\left\{e_{n_{l}^{\prime}} \in E^{1} \mid \text { there exists } e=\left(e_{n_{l}^{\prime}}, \ldots, e_{1}\right) \in \operatorname{supp}\left(\xi_{l}^{\prime \prime}\right)\right\} \\
\cup \bigcup_{m_{l}^{\prime} \geq 1}\left\{e_{m_{l}^{\prime}} \in E^{1} \mid \text { there exists } e=\left(e_{m_{l}^{\prime}}, \ldots, e_{1}\right) \in \operatorname{supp}\left(\eta_{l}^{\prime \prime}\right)\right\},
\end{aligned}
$$

which is a compact subset of $E^{1}$. Since $v \notin E_{\text {fin }}^{0}$, we have $r^{-1}(V) \backslash K \neq \emptyset$. Take an open set $U \subset r^{-1}(V) \backslash K$ such that the restriction of $d$ to $U$ is injective. Let $\zeta \in C_{c}(U) \subset C_{d}\left(E^{1}\right)$ be an element with $0 \leq \zeta \leq 1$ and $\zeta(e)=1$ for some $e \in U$. We have $\|\zeta\|=1$. We get $T^{1}(\zeta)^{*} T^{n_{l}^{\prime}}\left(\xi_{l}^{\prime \prime}\right) T^{m_{l}^{\prime}}\left(\eta_{l}^{\prime \prime}\right)^{*} T^{1}(\zeta)=0$ if either $n_{l}^{\prime}$ or $m_{l}^{\prime}$ is not zero. We set $a=T^{k}(\xi) T^{1}(\zeta), b=T^{k}(\eta) T^{1}(\zeta)$ and $f^{\prime}=\left\langle\zeta, \pi_{r}(g) \zeta\right\rangle$. Then we have $\|a\|,\|b\| \leq 1$ and

$$
\begin{aligned}
\left\|a^{*} x b-T^{0}\left(f^{\prime}\right)\right\| & =\left\|T^{1}(\zeta)^{*} y T^{1}(\zeta)-T^{1}(\zeta)^{*} T^{0}(g) T^{1}(\zeta)\right\| \\
& =\left\|T^{1}(\zeta)^{*} y T^{1}(\zeta)-T^{1}(\zeta)^{*} y^{\prime} T^{1}(\zeta)\right\| \leq\left\|y-y^{\prime}\right\|<\varepsilon-\delta .
\end{aligned}
$$

For $e^{\prime} \in U$, we have

$$
\left|f^{\prime}\left(d\left(e^{\prime}\right)\right)\right|=\left|\overline{\zeta\left(e^{\prime}\right)} g\left(r\left(e^{\prime}\right)\right) \zeta\left(e^{\prime}\right)\right| \leq\left|g\left(r\left(e^{\prime}\right)\right)\right|<\left\|x_{0}\right\|+\delta
$$

because the restriction of $d$ to $U$ is injective. We also have

$$
\left|f^{\prime}(d(e))\right|=|\overline{\zeta(e)} g(r(e)) \zeta(e)|=|g(r(e))|>\left\|x_{0}\right\|-\delta .
$$

Hence we get $\left|\left\|f^{\prime}\right\|-\left\|x_{0}\right\|\right|<\delta$. Therefore $f=\left\|x_{0}\right\| f^{\prime} /\left\|f^{\prime}\right\|$ satisfies $\|f\|=\left\|x_{0}\right\|$ and $\left\|f-f^{\prime}\right\|<\delta$. Thus we have

$$
\left\|a^{*} x b-T^{0}(f)\right\| \leq\left\|a^{*} x b-T^{0}\left(f^{\prime}\right)\right\|+\left\|f^{\prime}-f\right\|<\varepsilon .
$$

Case 2, $k=n$. Next we consider the case that $\left\|x_{0}\right\|=\left\|\pi_{n}^{n}\left(x_{0}\right)\right\|$. We can find $\overline{\xi, \eta \in C_{d}\left(E^{n}\right)}$ with $\|\xi\|=\|\eta\|=1$ such that $\left\|\left\langle\xi, \pi_{n}^{n}\left(x_{0}\right) \eta\right\rangle\right\|>\left\|x_{0}\right\|-\varepsilon$. Set $g=\left\langle\xi, \pi_{n}^{n}\left(x_{0}\right) \eta\right\rangle \in C_{0}\left(E^{0}\right)$. There exists a non-empty open set $V$ of $E^{0}$ such that $|g(v)|>\left\|x_{0}\right\|-\varepsilon$ for $v \in V$. When $n_{l}>m_{l}$, we have

$$
T^{n}(\xi)^{*} T^{n_{l}}\left(\xi_{l}\right) T^{m_{l}}\left(\eta_{l}\right)^{*} T^{n}(\eta)=T^{n_{l}^{\prime}}\left(\xi_{l}^{\prime}\right)
$$

for some $\xi_{l}^{\prime} \in C_{d}\left(E^{n_{l}^{\prime}}\right)$ where $n_{l}^{\prime}=n_{l}-m_{l}$. Similarly when $n_{l}<m_{l}$, we have

$$
T^{n}(\xi)^{*} T^{n_{l}}\left(\xi_{l}\right) T^{m_{l}}\left(\eta_{l}\right)^{*} T^{n}(\eta)=T^{m_{l}^{\prime}}\left(\eta_{l}^{\prime}\right)^{*}
$$

for some $\eta_{l}^{\prime} \in C_{d}\left(E^{m_{l}^{\prime}}\right)$ where $m_{l}^{\prime}=m_{l}-n_{l}$. We have

$$
T^{n}(\xi)^{*} x T^{n}(\eta)=T^{0}(g)+\sum_{n_{l}>m_{l}} T^{n_{l}^{\prime}}\left(\xi_{l}^{\prime}\right)+\sum_{n_{l}<m_{l}} T^{m_{l}^{\prime}}\left(\eta_{l}^{\prime}\right)^{*} .
$$

Subcase 2.1, $\left(r^{n+1}\right)^{-1}(V)=\emptyset$. Take an integer $k$ with $0 \leq k \leq n$ satisfying $\left(r^{k}\right)^{-1}(V)$ $\neq \emptyset$ and $\left(r^{k+1}\right)^{-1}(V)=\emptyset$. Take $\zeta \in C_{c}\left(\left(r^{k}\right)^{-1}(V)\right) \subset C_{d}\left(E^{k}\right)$ such that $\|\zeta\|=1$ and $\zeta(e)=1$ for some $e \in\left(r^{k}\right)^{-1}(V)$. Set $a=T^{n}(\xi) T^{k}(\zeta)$ and $b=T^{n}(\eta) T^{k}(\zeta)$. Then we have $\|a\|,\|b\| \leq 1$. We see that

$$
T^{k}(\zeta)^{*} T^{n_{l}^{\prime}}\left(\xi_{l}^{\prime}\right) T^{k}(\zeta)=0 \quad \text { and } \quad T^{k}(\zeta)^{*} T^{m_{l}^{\prime}}\left(\eta_{l}^{\prime}\right)^{*} T^{k}(\zeta)=0
$$

by Lemma 5.8 Hence we get

$$
a^{*} x b=T^{k}(\zeta)^{*} T^{0}(g) T^{k}(\zeta)
$$


Set $f^{\prime}=\left\langle\zeta, \pi_{r^{k}}(g) \zeta\right\rangle$. Then we have $\left\|f^{\prime}\right\| \leq\|g\| \leq\left\|x_{0}\right\|$ and

$$
\left\|f^{\prime}\right\| \geq\left|f^{\prime}(d(e))\right| \geq\left|\overline{\zeta(e)} g\left(r^{k}(e)\right) \zeta(e)\right| \geq\left\|x_{0}\right\|-\varepsilon .
$$

Therefore we get $\left|\left\|f^{\prime}\right\|-\left\|x_{0}\right\|\right|<\varepsilon$. Hence $f=\left\|x_{0}\right\| f^{\prime} /\left\|f^{\prime}\right\|$ satisfies $\|f\|=\left\|x_{0}\right\|$ and $\left\|a^{*} x b-T^{0}(f)\right\|<\varepsilon$.

Subcase 2.2, $\left(r^{n+1}\right)^{-1}(V) \neq \emptyset$. By Lemma $[5.9$ there exists a non-returning path $e \in E^{m}$ with $m \geq n+1$ and $r(e) \in V$. By Lemma [5.6, we can find a non-empty open set $U \subset\left(r^{m}\right)^{-1}(V) \subset E^{m}$ which is non-returning. Choose $\zeta \in C_{c}(U) \subset$ $C_{d}\left(E^{m}\right)$ such that $\|\zeta\|=1$ and $\zeta(e)=1$ for some $e \in U$. Set $a=T^{n}(\xi) T^{m}(\zeta)$, $b=T^{n}(\eta) T^{m}(\zeta)$ and $f^{\prime}=\left\langle\zeta, \pi_{r^{m}}(g) \zeta\right\rangle$. Then we have $\|a\|,\|b\| \leq 1$ and $a^{*} x b=$ $T^{m}(\zeta)^{*} y T^{m}(\zeta)=T^{0}\left(f^{\prime}\right)$ by Lemma 5.7 Similarly to the proof in Subcase 2.1, we have $\left|\left\|f^{\prime}\right\|-\left\|x_{0}\right\|\right|<\varepsilon$. Hence $f=\left\|x_{0}\right\| f^{\prime} /\left\|f^{\prime}\right\|$ satisfies $\|f\|=\left\|x_{0}\right\|$ and $\left\|a^{*} x b-T^{0}(f)\right\|<\varepsilon$.

The proof is completed.

Remark 5.11. For a positive element $x \in \mathcal{L}(X)$ where $X$ is a Hilbert module, we have $\langle\xi, x \xi\rangle \geq 0$ for any $\xi \in X$ and $\|x\|=\sup _{\|\xi\|=1}\|\langle\xi, x \xi\rangle\|$. Therefore if we further assume that $x_{0} \geq 0$ in the hypothesis of Proposition [5.10, then we can take $a, b \in C^{*}(T)$ and $f \in C_{0}\left(E^{0}\right)$ in the conclusion there so that $a=b$ and $f \geq 0$ because we can take $\xi=\eta$ in both Case 1 and Case 2 in the above proof. We will use this fact in $\mathrm{Ka} 4$.

The following is our version of the Cuntz-Krieger uniqueness theorem.

Theorem 5.12. If a topological graph $E=\left(E^{0}, E^{1}, d, r\right)$ is topologically free, then the map $\rho_{T}: \mathcal{O}(E) \rightarrow C^{*}(T)$ is an isomorphism for any injective Cuntz-Krieger E-pair $T=\left(T^{0}, T^{1}\right)$.

Proof. We check the condition (iii) in Theorem 4.5. By Proposition 5.10 for any $\varepsilon>0$ we have $\left\|x_{0}\right\|=\|f\| \leq\left\|a^{*} x b\right\|+\varepsilon \leq\|x\|+\varepsilon$ for $x=\sum_{l=1}^{L} T^{n_{l}}\left(\xi_{l}\right) T^{m_{l}}\left(\eta_{l}\right)^{*}$ and $x_{0}=\sum_{n_{l}=m_{l}} T^{n_{l}}\left(\xi_{l}\right) T^{m_{l}}\left(\eta_{l}\right)^{*}$ where $\xi_{l} \in C_{d}\left(E^{n_{l}}\right)$ and $\eta_{l} \in C_{d}\left(E^{m_{l}}\right)$. Hence $x \mapsto x_{0}$ gives us a well-defined norm-decreasing linear map from

$$
\operatorname{span}\left\{T^{n}(\xi) T^{m}(\eta)^{*} \mid \xi \in C_{d}\left(E^{n}\right), \eta \in C_{d}\left(E^{m}\right), n, m \in \mathbb{N}\right\}
$$

to $\mathcal{F}_{T} \subset C^{*}(T)$. It extends a linear map $\Psi_{T}$ from $C^{*}(T)$ to $\mathcal{F}_{T}$ which is the identity on $\mathcal{F}_{T}$. Hence $\Psi_{T}$ is a conditional expectation onto $\mathcal{F}_{T}$ such that $\Psi_{T}\left(T^{n}(\xi) T^{m}(\eta)^{*}\right)$ $=\delta_{n, m} T^{n}(\xi) T^{m}(\eta)^{*}$ for $\xi \in C_{d}\left(E^{n}\right), \eta \in C_{d}\left(E^{m}\right)$. By Theorem 4.5, $\rho_{T}$ is an isomorphism.

Remark 5.13. In the theorem above, the assumption that a topological graph $E$ is topologically free is needed. When $E$ is not topologically free, there exists an injective Cuntz-Krieger $E$-pair $T$ such that the map $\rho_{T}: \mathcal{O}(E) \rightarrow C^{*}(T)$ is not an isomorphism (see [Ka3]).

\section{6. $K K$-groups of $\mathcal{O}(E)$}

In this section, we prove that our $C^{*}$-algebras $\mathcal{O}(E)$ are always nuclear and satisfy the Universal Coefficient Theorem (UCT) of [RoSc], and compute their $K K$-groups. To this end, we need a short exact sequence which is (almost) established in Section 3 Take a topological graph $E=\left(E^{0}, E^{1}, d, r\right)$. In Section 3] we defined a Toeplitz $E$-pair $\sigma$ on $\mathcal{L}\left(C_{d}\left(E^{*}\right)\right)$ which was shown to be universal (Proposition [3.8). Hence we can identify $C^{*}(\sigma)$ with $\mathcal{T}(E)$. The $C^{*}$-algebra $\mathcal{T}(E)=C^{*}(\sigma)$ has an ideal 
$\mathcal{K}\left(C_{d}\left(E_{\mathrm{rg}}^{*}\right)\right)$ which we will denote $I_{E}$. The quotient $\mathcal{T}(E) / I_{E}$ is the $C^{*}$-algebra $C^{*}(\tau)$ which was shown to be isomorphic to the $C^{*}$-algebra $\mathcal{O}(E)$ in Corollary 4.7 Hence we have the following short exact sequence:

$$
0 \longrightarrow I_{E} \stackrel{j}{\longrightarrow} \mathcal{T}(E) \longrightarrow \mathcal{O}(E) \longrightarrow 0 .
$$

Proposition 6.1. The $C^{*}$-algebra $\mathcal{O}(E)$ is nuclear.

Proof. The $C^{*}$-algebra $\mathcal{T}(E)$ is isomorphic to the augmented Toeplitz algebra $\widetilde{\mathcal{T}}_{C_{d}\left(E^{1}\right)}$. There is a folklore 1 that an augmented Toeplitz algebra $\widetilde{\mathcal{T}}_{X}$ of a $C^{*}$ correspondences $X$ over $A$ is nuclear if and only if $A$ is nuclear (the proof goes similarly as in [DS]). Hence the $C^{*}$-algebra $\widetilde{\mathcal{T}}_{C_{d}\left(E^{1}\right)}$ is nuclear because $C_{0}\left(E^{0}\right)$ is nuclear. Since nuclearity inherits to quotients (see, for example, [W] Corollary $2.5])$, the $C^{*}$-algebra $\mathcal{O}(E)$ is nuclear.

It is well known that a $C^{*}$-algebra $C_{0}\left(E^{0}\right)$ is separable if and only if $E^{0}$ is second countable. Similarly we have the following.

Lemma 6.2. The Banach space $C_{d}\left(E^{1}\right)$ is separable if and only if $E^{1}$ is second countable.

Proof. If $\left\{\xi_{k}\right\}_{k \in \mathbb{N}}$ is a countable dense set of $C_{d}\left(E^{1}\right)$, then $\left\{U_{k}\right\}_{k \in \mathbb{N}}$ is a countable open basis of $E^{1}$ where $U_{k}=\left\{e \in E^{1}|| \xi_{k}(e) \mid<1\right\}$. Hence if $C_{d}\left(E^{1}\right)$ is separable, $E^{1}$ is second countable. Conversely if $E^{1}$ is second countable, we can find a countable subset $X$ of $C_{c}\left(E^{1}\right)$ such that for every open subset $U$ of $E^{1}$, every elements of $C_{c}(U)$ can be uniformly approximated by elements of $X \cap C_{c}(U)$. By Lemma 1.26 we see that the countable subset $X$ is dense in $C_{d}\left(E^{1}\right)$ with respect to the norm $\|\cdot\|$. Hence $C_{d}\left(E^{1}\right)$ is separable.

We say that a topological graph $E=\left(E^{0}, E^{1}, d, r\right)$ is second countable if both $E^{0}$ and $E^{1}$ are second countable.

Proposition 6.3. The $C^{*}$-algebra $\mathcal{O}(E)$ is separable if and only if $E$ is second countable.

Proof. If $\mathcal{O}(E)$ is separable, then both $C_{0}\left(E^{0}\right)$ and $C_{d}\left(E^{1}\right)$ are separable because $t^{0}$ and $t^{1}$ are isometric. Conversely if both $C_{0}\left(E^{0}\right)$ and $C_{d}\left(E^{1}\right)$ are separable, then $\mathcal{O}(E)$ is separable because $\mathcal{O}(E)$ is generated by the images of $C_{0}\left(E^{0}\right)$ and $C_{d}\left(E^{1}\right)$. Now the proof ends by Lemma 6.2.

In the rest of this section, we assume that $E$ is second countable.

Lemma 6.4. The Hilbert $C_{0}\left(E_{\mathrm{rg}}^{0}\right)$-module $C_{d}\left(E_{\mathrm{rg}}^{*}\right)$ is full. Hence it gives a strong Morita equivalence between $I_{E}=\mathcal{K}\left(C_{d}\left(E_{\mathrm{rg}}^{*}\right)\right)$ and $C_{0}\left(E_{\mathrm{rg}}^{0}\right)$.

Proof. It is easy to verify.

We denote by $\left[C_{d}\left(E_{\mathrm{rg}}^{*}\right)\right] \in K K\left(I_{E}, C_{0}\left(E_{\mathrm{rg}}^{0}\right)\right)$ the element defined by the imprimitivity bimodule $C_{d}\left(E_{\mathrm{rg}}^{*}\right)$. This element gives a $K K$-equivalence of $I_{E}$ and $C_{0}\left(E_{\mathrm{rg}}^{0}\right)$. Next we see that the inclusion $\sigma^{0}: C_{0}\left(E^{0}\right) \rightarrow \mathcal{T}(E)$ gives a $K K$-equivalence from $C_{0}\left(E^{0}\right)$ to $\mathcal{T}(E)$ following $\left[\mathrm{P}\right.$. We define a graded Kasparov module $\left(C_{d}\left(E^{*}\right) \oplus\right.$ $\left.C_{d}\left(E^{*}\right), \pi \oplus \pi_{+}, T\right)$ as follows. A Toeplitz $E$-pair $\sigma$ on $\mathcal{L}\left(C_{d}\left(E^{*}\right)\right)$ gives an injective map $\pi: \mathcal{T}(E) \rightarrow \mathcal{L}\left(C_{d}\left(E^{*}\right)\right)$. Define $E_{+}^{*}=\bigsqcup_{n=1}^{\infty} E^{n}$ which is an open and closed

\footnotetext{
${ }^{1}$ In [Ka5], we give a proof of this folklore.
} 
subset of $E^{*}$. Hence $C_{d}\left(E_{+}^{*}\right)$ is a direct summand of $C_{d}\left(E^{*}\right)$ and we can consider $\mathcal{L}\left(C_{d}\left(E_{+}^{*}\right)\right) \subset \mathcal{L}\left(C_{d}\left(E^{*}\right)\right)$. This summand $C_{d}\left(E_{+}^{*}\right)$ is closed under the maps $\sigma^{0}, \sigma^{1}$. We write the restrictions of $\sigma^{0}, \sigma^{1}$ to $C_{d}\left(E_{+}^{*}\right)$ by $\sigma_{+}^{0}, \sigma_{+}^{1}$ respectively. Since the pair $\sigma_{+}=\left(\sigma_{+}^{0}, \sigma_{+}^{1}\right)$ is a restriction of a Toeplitz $E$-pair $\sigma=\left(\sigma^{0}, \sigma^{1}\right)$, it is also a Toeplitz $E$-pair on $\mathcal{L}\left(C_{d}\left(E_{+}^{*}\right)\right)$. This gives a $*$-homomorphism $\pi_{+}: \mathcal{T}(E) \rightarrow \mathcal{L}\left(C_{d}\left(E_{+}^{*}\right)\right)$. Since $\mathcal{L}\left(C_{d}\left(E_{+}^{*}\right)\right) \subset \mathcal{L}\left(C_{d}\left(E^{*}\right)\right)$, we regard $\pi_{+}$as a $*$-homomorphism to $\mathcal{L}\left(C_{d}\left(E^{*}\right)\right)$. Define an odd operator $T \in \mathcal{L}\left(C_{d}\left(E^{*}\right) \oplus C_{d}\left(E^{*}\right)\right)$ by $T(\xi \oplus \eta)=\eta \oplus \xi$. By $[\mathrm{P}$ Lemma 4.2], the triple $\left(C_{d}\left(E^{*}\right) \oplus C_{d}\left(E^{*}\right), \pi \oplus \pi_{+}, T\right)$ is a Kasparov module, and so it gives an element $\beta$ in $K K\left(\mathcal{T}(E), C_{0}\left(E^{0}\right)\right)$.

Lemma 6.5. The element $\beta \in K K\left(\mathcal{T}(E), C_{0}\left(E^{0}\right)\right)$ is the inverse of the element $\sigma_{*}^{0} \in K K\left(C_{0}\left(E^{0}\right), \mathcal{T}(E)\right)$. Hence $\mathcal{T}(E)$ is $K K$-equivalent to $C_{0}\left(E^{0}\right)$.

Proof. See [P, Theorem 4.4].

Proposition 6.6. For a second countable topological graph $E$, the $C^{*}$-algebra $\mathcal{O}(E)$ satisfies the UCT.

Proof. Since $I_{E}$ and $\mathcal{T}(E)$ are $K K$-equivalent to commutative $C^{*}$-algebras $C_{0}\left(E_{\mathrm{rg}}^{0}\right)$ and $C_{0}\left(E^{0}\right)$ respectively, they satisfy the UCT. Now "two among three principle" shows that $\mathcal{O}(E)$ satisfies the UCT.

Finally we compute the $K K$-groups of $\mathcal{O}(E)$ in terms of the topological graph $E$. To do so, we examine the element $j_{*} \in K K\left(I_{E}, \mathcal{T}(E)\right)$ defined by the inclusion $j: I_{E} \rightarrow \mathcal{T}(E)$. We denote by $\left[\pi_{r}\right]$ the element of $K K\left(C_{0}\left(E_{\mathrm{rg}}^{0}\right), C_{0}\left(E^{0}\right)\right)$ defined by a triple $\left(C_{d}\left(E^{1}\right), \pi_{r}, 0\right)$ and denote by $\iota: C_{0}\left(E_{\mathrm{rg}}^{0}\right) \rightarrow C_{0}\left(E^{0}\right)$ a natural embedding. We have the following.

Lemma 6.7. With the notation above, we have

$$
\left[C_{d}\left(E_{\mathrm{rg}}^{*}\right)\right] \otimes_{C_{0}\left(E_{\mathrm{rg}}^{\mathrm{o}}\right)}\left(\iota_{*}-\left[\pi_{r}\right]\right)=j_{*} \otimes_{\mathcal{T}(E)} \beta
$$

in $K K\left(I_{E}, C_{0}\left(E^{0}\right)\right)$.

Proof. The proof is exactly the same as in [P, Lemma 4.7], hence we omit it.

Remark 6.8. When $E^{1}=E^{0}$ and $d=\mathrm{id}$, there exists a natural isomorphism $\mathcal{K}\left(C_{d}\left(E^{1}\right)\right) \cong C_{0}\left(E^{0}\right)$. Under this isomorphism, the restriction of $\pi_{r}$ to $C_{0}\left(E_{\mathrm{rg}}^{0}\right)$ coincides with a $*$-homomorphism $\hat{r}: C_{0}\left(E_{\mathrm{rg}}^{0}\right) \ni f \mapsto f \circ r \in C_{0}\left(E^{0}\right)$. We see that the element $\left[\pi_{r}\right] \in K K\left(C_{0}\left(E_{\mathrm{rg}}^{0}\right), C_{0}\left(E^{0}\right)\right)$ is defined by this $*$-homomorphism $\hat{r}$. For a general topological graph $E, \mathcal{K}\left(C_{d}\left(E^{1}\right)\right)$ is strongly Morita equivalent to the ideal $C_{0}\left(d\left(E^{1}\right)\right)$ of $C_{0}\left(E^{0}\right)$ via the Hilbert module $C_{d}\left(E^{1}\right)$. Thus we have an element of $\left[C_{d}\left(E^{1}\right)\right] \in K K\left(\mathcal{K}\left(C_{d}\left(E^{1}\right)\right), C_{0}\left(E^{0}\right)\right)$. The element $\left[\pi_{r}\right] \in K K\left(C_{0}\left(E_{\mathrm{rg}}^{0}\right), C_{0}\left(E^{0}\right)\right)$ is the Kasparov product of the element $\left(\pi_{r}\right)_{*} \in K K\left(C_{0}\left(E_{\mathrm{rg}}^{0}\right), \mathcal{K}\left(C_{d}\left(E^{1}\right)\right)\right)$ induced by $\pi_{r}: C_{0}\left(E_{\mathrm{rg}}^{0}\right) \rightarrow \mathcal{K}\left(C_{d}\left(E^{1}\right)\right)$ and $\left[C_{d}\left(E^{1}\right)\right]$.

Proposition 6.9. Let $E$ be a second countable topological graph. For any separable $C^{*}$-algebra $B$, we have the following two exact sequences:

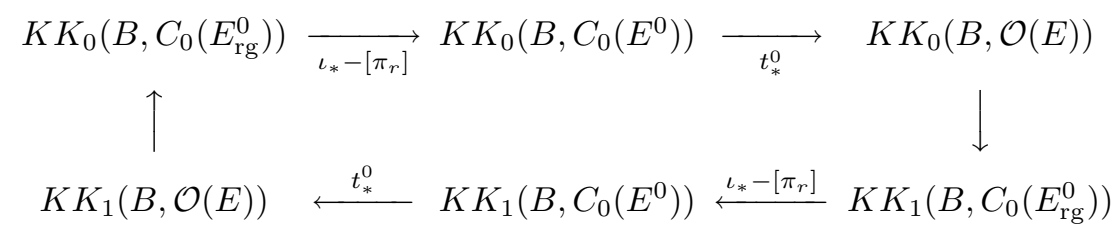


and

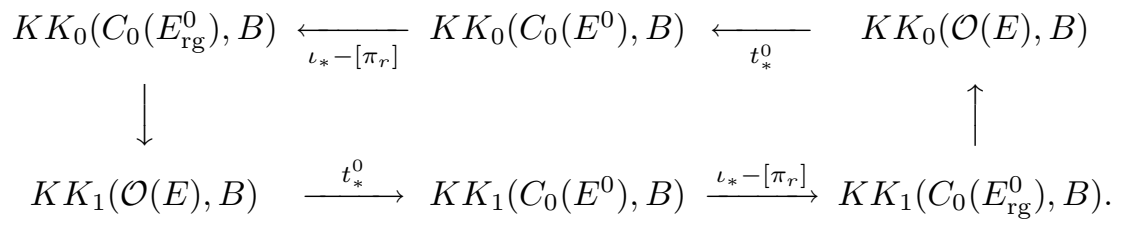

Proof. From the short exact sequence

$$
0 \longrightarrow I_{E} \stackrel{j}{\longrightarrow} \mathcal{T}(E) \longrightarrow \mathcal{O}(E) \longrightarrow 0,
$$

we have two 6-term exact sequences of $K K$-groups because $\mathcal{O}(E)$ is nuclear. As we saw above, $I_{E}$ and $\mathcal{T}(E)$ are $K K$-equivalent to $C_{0}\left(E_{\mathrm{rg}}^{0}\right)$ and $C_{0}\left(E^{0}\right)$ respectively. Under these isomorphisms, the element $j_{*} \in K K\left(I_{E}, \mathcal{T}(E)\right)$ coincides with $\iota_{*}-\left[\pi_{r}\right] \in K K\left(C_{0}\left(E_{\mathrm{rg}}^{0}\right), C_{0}\left(E^{0}\right)\right)$ by Lemma 6.7. The composition of the map $\sigma^{0}: C_{0}\left(E^{0}\right) \rightarrow \mathcal{T}(E)$ and the quotient map $\mathcal{T}(E) \rightarrow \mathcal{O}(E)$ is $t^{0}: C_{0}\left(E^{0}\right) \rightarrow \mathcal{O}(E)$. Now we have the desired 6 -term exact sequences.

Corollary 6.10. For a second countable topological graph E, we have the following exact sequence of $K$-groups:

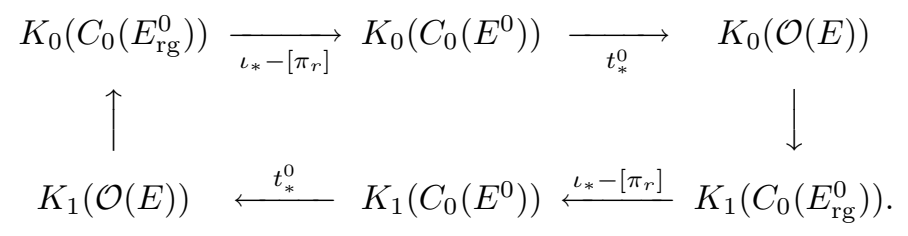

Finally we give a new proof of the computation of $K$-groups of graph algebras by using Corollary 6.10 Let $E=\left(E^{0}, E^{1}, d, r\right)$ be a discrete graph. The group $K_{0}\left(C_{0}\left(E^{0}\right)\right)$ is isomorphic to a free abelian group $\mathbb{Z}^{E^{0}}$ whose generators are $\left\{\left[\delta_{v}\right]\right\}_{v \in E^{0}}$, and $K_{1}\left(C_{0}\left(E^{0}\right)\right)=0$. We also have $K_{0}\left(C_{0}\left(E_{\mathrm{rg}}^{0}\right)\right) \cong \mathbb{Z}^{E_{\mathrm{rg}}^{0}}$ and $K_{1}\left(C_{0}\left(E_{\mathrm{rg}}^{0}\right)\right)=0$. For $v \in E_{\mathrm{rg}}^{0}$, we have $\iota_{*}\left(\left[\delta_{v}\right]\right)=\left[\delta_{v}\right]$. We will compute $\left[\pi_{r}\right]\left(\left[\delta_{v}\right]\right) \in K_{0}\left(C_{0}\left(E^{0}\right)\right)$. By the computation done in Section 2] we have $\pi_{r}\left(\delta_{v}\right)=$ $\sum_{e \in r^{-1}(v)} \theta_{\delta_{e}, \delta_{e}}$ for $v \in E_{\mathrm{rg}}^{0}$ (note that $r^{-1}(v)$ is a non-empty finite set). There exists an isomorphism

$$
\psi: K_{0}\left(\mathcal{K}\left(C_{d}\left(E^{1}\right)\right)\right) \rightarrow K_{0}\left(C_{0}\left(d\left(E^{1}\right)\right)\right) \cong \mathbb{Z}^{d\left(E^{1}\right)} \subset \mathbb{Z}^{E^{0}}
$$

defined by $\psi\left(\left[\theta_{\delta_{e}, \delta_{e}}\right]\right)=\left[\delta_{d(e)}\right]$ for $e \in E^{1}$. Using this map, we have

$$
\left[\pi_{r}\right]\left(\left[\delta_{v}\right]\right)=\psi\left(\left[\pi_{r}\left(\delta_{v}\right)\right]\right)=\psi\left(\sum_{e \in r^{-1}(v)}\left[\theta_{\delta_{e}, \delta_{e}}\right]\right)=\sum_{e \in r^{-1}(v)}\left[\delta_{d(e)}\right] \in K_{0}\left(C_{0}\left(E^{0}\right)\right) .
$$

Now Corollary 6.10 gives us the following.

Proposition 6.11 ([Sz, Proposition 2], [DT2, Theorem 3.1]). Let $E=\left(E^{0}, E^{1}, d, r\right)$ be a discrete graph. Define a homomorphism $\Delta: \mathbb{Z}^{E_{\mathrm{rg}}^{0}} \rightarrow \mathbb{Z}^{E^{0}}$ by $\Delta\left(\left[\delta_{v}\right]\right)=\left[\delta_{v}\right]-$ $\sum_{e \in r^{-1}(v)}\left[\delta_{d(e)}\right]$. Then we have isomorphisms $K_{0}(\mathcal{O}(E)) \cong$ coker $\Delta$ and $K_{1}(\mathcal{O}(E))$ $\cong \operatorname{ker} \Delta$.

\footnotetext{
${ }^{2}$ In [Ka5], we prove this corollary without the assumption of second countability.
} 


\section{ACKNOWLEDGMENTS}

The author is grateful to Yasuyuki Kawahigashi and Masaki Izumi for their encouragement, and to Iain Raeburn and Wojciech Szymański for stimulating discussions. He would also like to thank David Pask and Paul S. Muhly for valuable comments, and Valentin Deaconu for pointing out grammatical mistakes in the earlier draft of this paper. This work was completed while the author was staying at the University of Oregon. He would like to thank people there, especially Huaxin Lin, for their warm hospitality. This work was partially supported by Research Fellowship for Young Scientists of the Japan Society for the Promotion of Science.

\section{REFERENCES}

[AS] Archbold, R. J.; Spielberg, J. S. Topologically free actions and ideals in discrete $C^{*}$ dynamical systems. Proc. Edinburgh Math. Soc. (2) 37 (1994), no. 1, 119-124. MR 94m:46101

[AR] Arzumanian, V.; Renault, J. Examples of pseudogroups and their $C^{*}$-algebras. Operator algebras and quantum field theory, 93-104, Internat. Press, Cambridge, MA, 1997. MR 99a:46101

[BHRS] Bates, T.; Hong, J.; Raeburn, I.; Szymański, W. The ideal structure of the $C^{*}$-algebras of infinite graphs. Illinois J. Math. 46 (2002), no. 4, 1159-1176.

[BPRS] Bates, T.; Pask, D.; Raeburn, I.; Szymański, W. The $C^{*}$-algebras of row-finite graphs. New York J. Math. 6 (2000), 307-324. MR 2001k:46084

[CK] Cuntz, J.; Krieger, W. A class of $C^{*}$-algebras and topological Markov chains. Invent. Math. 56 (1980), no. 3, 251-268. MR 82f:46073a

[D] Deaconu, V. Continuous graphs and $C^{*}$-algebras. Operator theoretical methods, 137149, Theta Found., Bucharest, 2000. MR 2001g:46123

[DKM] Deaconu, V.; Kumjian, A.; Muhly, P. Cohomology of topological graphs and CuntzPimsner algebras. J. Operator Theory 46 (2001), no. 2, 251-264. MR 2003a:46093

[DT1] Drinen, D.; Tomforde, M. The $C^{*}$-algebras of arbitrary graphs. To appear in Rocky Mountain J. Math.

[DT2] Drinen, D.; Tomforde, M. Computing $K$-theory and Ext for graph $C^{*}$-algebras. Illinois J. Math. 46 (2002), no. 1, 81-91. MR 2003k:46103

[DS] Dykema, K.; Shlyakhtenko, D. Exactness of Cuntz-Pimsner C $^{*}$-algebras. Proc. Edinburgh Math. Soc. 44 (2001), 425-444. MR 2003a:46084

[E] Exel, R. A Fredholm operator approach to Morita equivalence. K-Theory 7 (1993), no. 3, 285-308. MR 94h:46107

[EL] Exel, R.; Laca, M. Cuntz-Krieger algebras for infinite matrices. J. Reine Angew. Math. 512 (1999), 119-172. MR 2000i:46064

[ELQ] Exel, R.; Laca, M.; Quigg, J. Partial dynamical systems and $C^{*}$-algebras generated by partial isometries. J. Operator Theory 47 (2002), no. 1, 169-186. MR 2003f:46108

[FLR] Fowler, N. J.; Laca, M.; Raeburn, I. The $C^{*}$-algebras of infinite graphs. Proc. Amer. Math. Soc. 128 (2000), no. 8, 2319-2327. MR 2000k:46079

[FR] Fowler, N. J.; Raeburn, I. The Toeplitz algebra of a Hilbert bimodule. Indiana Univ. Math. J. 48 (1999), no. 1, 155-181. MR 2001b:46093

[HS] Hong, J. H.; Szymanski, W. The primitive ideal space of the $C^{*}$-algebras of infinite graphs. J. Math. Soc. Japan 56 (2004), no. 1, 45-64.

[KPW] Kajiwara, T.; Pinzari, C.; Watatani, Y. Ideal structure and simplicity of the $C^{*}$ algebras generated by Hilbert bimodules. J. Funct. Anal. 159 (1998), no. 2, 295-322. MR 2000a:46094

[Ka1] Katsura, T. The ideal structures of crossed products of Cuntz algebras by quasi-free actions of abelian groups. Canad. J. Math. 55 (2003), no. 6, 1302-1338.

[Ka2] Katsura, T. A class of $C^{*}$-algebras generalizing both graph algebras and homeomorphism $C^{*}$-algebras II, examples. Preprint 2004, math.OA/0405268.

[Ka3] Katsura, T. A class of $C^{*}$-algebras generalizing both graph algebras and homeomorphism $C^{*}$-algebras III, ideal structures. In preparation. 
[Ka4] Katsura, T. A class of $C^{*}$-algebras generalizing both graph algebras and homeomorphism $C^{*}$-algebras $I V$, pure infiniteness. In preparation.

[Ka5] Katsura, T. On $C^{*}$-algebras associated with $C^{*}$-correspondences. Preprint 2003, math.OA/0309088, to appear in J. Funct. Anal.

[KPR] Kumjian, A.; Pask, D.; Raeburn, I. Cuntz-Krieger algebras of directed graphs. Pacific J. Math. 184 (1998), no. 1, 161-174. MR 99i:46049

[KPRR] Kumjian, A.; Pask, D.; Raeburn, I.; Renault, J. Graphs, groupoids, and Cuntz-Krieger algebras. J. Funct. Anal. 144 (1997), no. 2, 505-541. MR 98g:46083

[L] Lance, E. C. Hilbert $C^{*}$-modules. A toolkit for operator algebraists. London Mathematical Society Lecture Note Series, 210. Cambridge University Press, Cambridge, 1995. MR 96k:46100

[MS] Muhly, P. S.; Solel, B. Tensor algebras over $C^{*}$-correspondences: representations, dilations, and $C^{*}$-envelopes. J. Funct. Anal. 158 (1998), no. 2, 389-457. MR 99j:46066

[P] Pimsner, M. V. A class of $C^{*}$-algebras generalizing both Cuntz-Krieger algebras and crossed products by $Z$. Free probability theory, 189-212, Fields Inst. Commun., 12, Amer. Math. Soc., Providence, RI, 1997. MR 97k:46069

[RaSz] Raeburn, I.; Szymański, W. Cuntz-Krieger algebras of infinite graphs and matrices. Preprint.

[RoSc] Rosenberg, J.; Schochet, C. The Kunneth theorem and the universal coefficient theorem for Kasparov's generalized K-functor. Duke Math. J. 55 (1987), no. 2, 431-474. MR 88i:46091

[Sc] Schweizer, J. Crossed products by $C^{*}$-correspondences and Cuntz-Pimsner algebras. $C^{*}$ algebras, 203-226, Springer, Berlin, 2000. MR 2002f:46133

[Sz] Szymański, W. On semiprojectivity of $C^{*}$-algebras of directed graphs. Proc. Amer. Math. Soc. 130 (2002), no. 5, 1391-1399. MR 2003a:46083

[T1] Tomiyama, J. The interplay between topological dynamics and theory of $C^{*}$-algebras. Lecture Notes Series, 2. Seoul National University, Research Institute of Mathematics, Global Analysis Research Center, Seoul, 1992. MR 93h:46097

[T2] Tomiyama, J. Structure of ideals and isomorphisms of $C^{*}$-crossed products by single homeomorphism. Tokyo J. Math. 23 (2000), no. 1, 1-13. MR 2001e:46117

[T3] Tomiyama, J. Hulls and kernels from topological dynamical systems and their applications to homeomorphism $C^{*}$-algebras. To appear in J. Math. Soc. Japan.

[T4] Tomiyama, J. On the projection theorem for homeomorphism $C^{*}$-algebras. Preprint.

[W] Wassermann, S. Exact $C^{*}$-algebras and related topics. Lecture Notes Series, 19. Seoul National University, Research Institute of Mathematics, Global Analysis Research Center, Seoul, 1994. MR 95b:46081

Department of Mathematical Sciences, University of Tokyo, Komaba, Tokyo 1538914, JAPAN

Current address: Department of Mathematics, Hokkaido University, Sapporo, Hokkaido 0600810, Japan

E-mail address: katsura@math.sci.hokudai.ac.jp 